\title{
Enantioselective Construction of Cyclobutanes: A New and Concise Approach to the Total Synthesis of Piperarborenine B
}

Jiang-Lin Hu, ${ }^{\dagger}$ Liang-Wen Feng, ${ }^{\dagger}$ Lijia Wang, ${ }^{\dagger}$ Zuowei Xie,,${ }^{*}{ }^{\S}$ Yong Tang,,${ }^{, \dagger}$ Xiaoge $\mathrm{Li}^{\dagger}$

${ }^{\dagger}$ The State Key Laboratory of Organometallic Chemistry and Shanghai-Hong Kong Joint Laboratory in Chemical Synthesis, Shanghai Institute of Organic Chemistry, Chinese Academy of Sciences, 345 Lingling Road, Shanghai 200032, China.

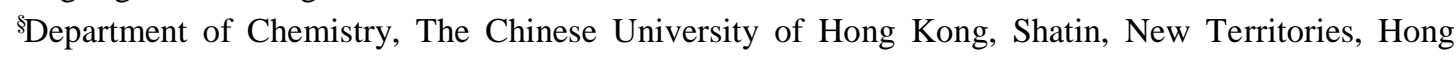
Kong, China

\section{tangy@mail.sioc.ac.cn}

zxie@cuhk.edu.hk

\section{Supporting Information}

\section{Table of Contents}

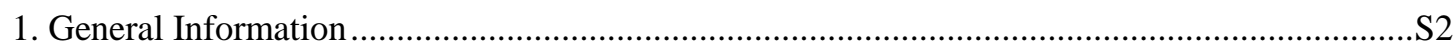

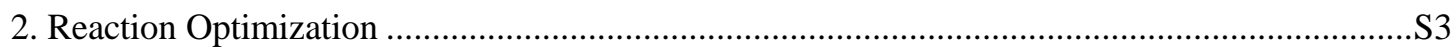

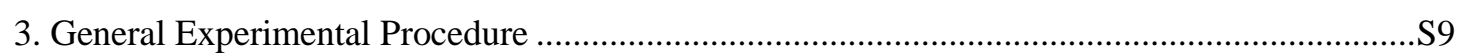

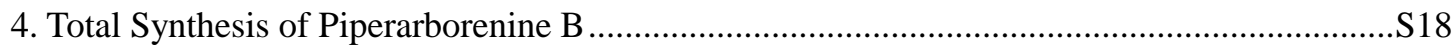

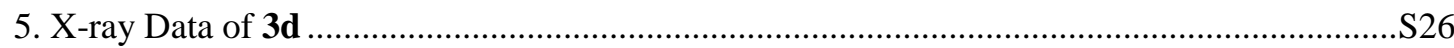

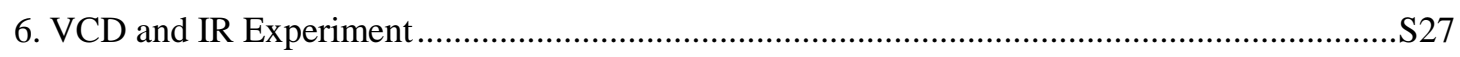

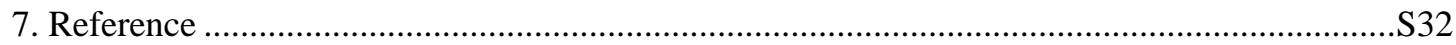

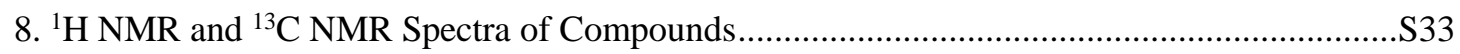

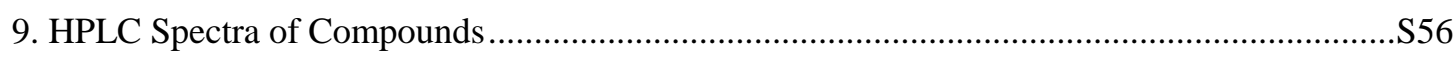




\section{General Information}

All reactions were carried out under dry nitrogen atmosphere. All solvents and reagents were obtained from commercial sources and were purified according to standard procedures. Molecular sieves powder (4 A MS and $5 \AA$ MS) were dried at $220{ }^{\circ} \mathrm{C}$ for 10 hours in vacuum before use. All glassware were oven dried before use.

${ }^{1} \mathrm{H}$ NMR spectra were recorded on a VARIAN Mercury $400 \mathrm{MHz}$ or Agilent 400 $\mathrm{MHz}$ spectrometer in chloroform-d. All signals are reported in ppm with the internal TMS signal at $0.0 \mathrm{ppm}$ or chloroform signal at $7.26 \mathrm{ppm}$ as a standard. The data are reported as $(\mathrm{s}=$ singlet, $\mathrm{d}=$ doublet, $\mathrm{t}=$ triplet, $\mathrm{q}=$ quadruplet, $\mathrm{m}=$ multiplet or unresolved, coupling constant(s) in $\mathrm{Hz}$, integration). ${ }^{13} \mathrm{C}$ NMR spectra were recorded on a VARIAN Mercury $100 \mathrm{MHz}$, or Agilent $100 \mathrm{MHz}$ spectrometer in chloroform-d. All signals are reported in ppm with the internal chloroform signal at $77.0 \mathrm{ppm}$ as a standard. IR spectra were recorded on a Perkin-Elmer 983, Digital FT-IR spectrometer or Bruker-Tensor 27; frequencies are given in reciprocal centimeters $\left(\mathrm{cm}^{-1}\right)$ and only selected absorbance is reported; Mass spectra were determined on an Agilent 5973N MSD (EI) and Shimadzu LC/MS-2010EV (ESI) mass spectrometer or Agilent G6100 LC/MSD (ESI) single Quand mass spectrometer. High resolution mass spectra were recorded on Waters Micromass GCT Premier (EI) and Bruker Daltonics, Inc. APEXIII 7.0 TESLA FTMS (ESI) mass spectrometers.

The Substrate $\mathbf{2 a}, \mathbf{2 d}$ and $\mathbf{2 g}$ were purchased from chemical company (J\&K and TCI); Substrates $\mathbf{2 b}, \mathbf{2 c}, \mathbf{2 e}, \mathbf{2 f}, \mathbf{2 h}-\mathbf{2 k}$ and $\mathbf{2 0}$ were prepared through Wittig olefination according to literature ${ }^{\mathrm{S} 1}$; Substrates $\mathbf{2 l - 2 n}$ were prepared followed by literatures ${ }^{\mathrm{S} 2}$; The methylidenemalonate was prepared followed by the literature ${ }^{\mathrm{S} 3}$, and it was stored in glovebox under $-30{ }^{\circ} \mathrm{C}$; All the ligands were synthesized according to the literatures. ${ }^{\mathrm{S}}$ 


\section{Reaction Optimization}

\section{Table S1 Effect of Lewis Acids ${ }^{\text {a }}$.}

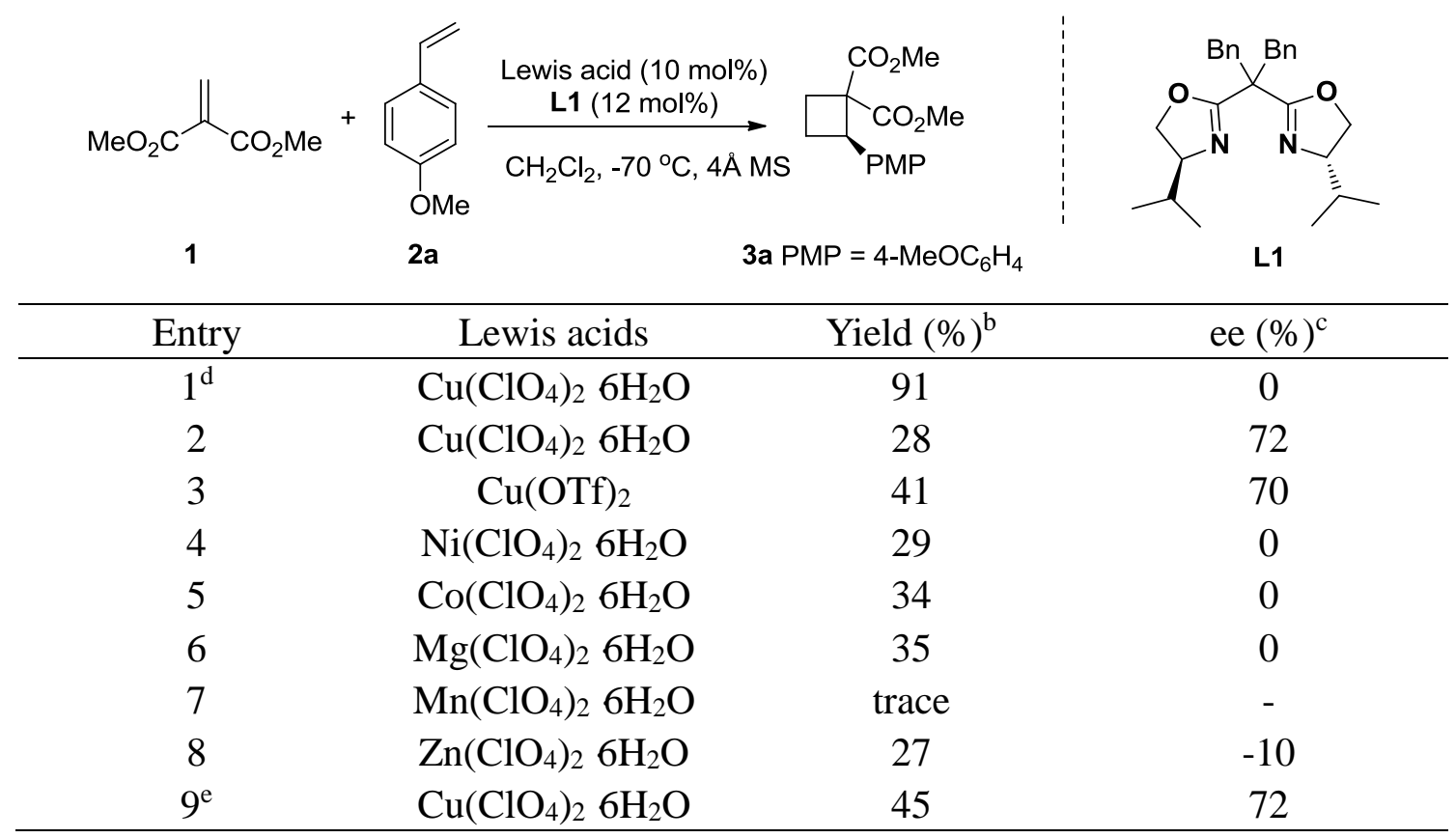

${ }^{a}$ Reaction conditions: Lewis acid (0.04 mmol), L1 (0.048 mmol), 4 Å MS (100 mg), 1 (1.0 mmol), and $\mathbf{2 a}(0.4 \mathrm{mmol})$ in $4.0 \mathrm{~mL}$ of $\mathrm{CH}_{2} \mathrm{Cl}_{2}$, After $\mathbf{1}$ or $\mathbf{2 a}$ was consumed, $\mathrm{Et}_{3} \mathrm{~N}$ was added to quench the reaction. ${ }^{b}$ Isolated yield. ${ }^{c}$ Determined by HPLC using a chiral stationary. ${ }^{d}$ Reaction performed at $0{ }^{\circ} \mathrm{C} .{ }^{e} \mathrm{Use} 5 \AA \mathrm{MS}$ as additive. 
Table S2 Effect of Solvent ${ }^{\mathrm{a}}$.

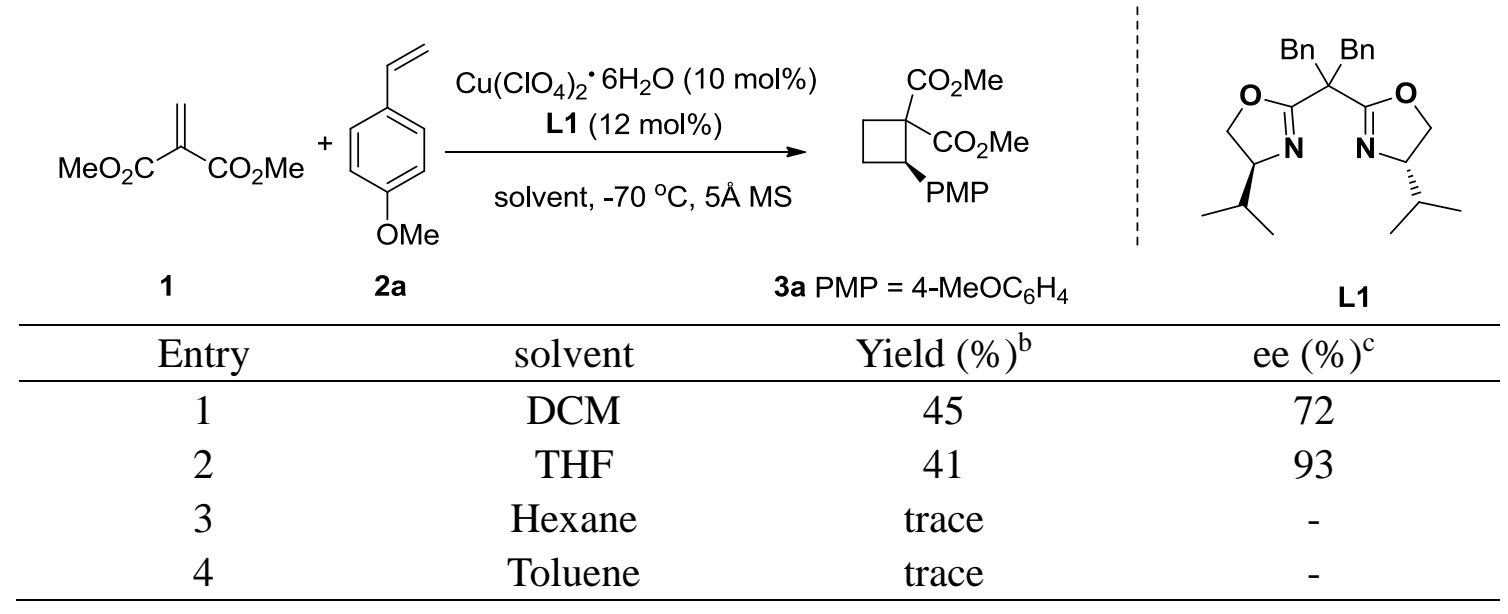

${ }^{a}$ Reaction conditions: $\mathrm{Cu}\left(\mathrm{ClO}_{4}\right)_{2} \cdot 6 \mathrm{H}_{2} \mathrm{O}(0.04 \mathrm{mmol}), \mathbf{L 1}(0.048 \mathrm{mmol}), 5 \AA \mathrm{MS}(100 \mathrm{mg}), \mathbf{1}(1.0$ mmol), and $\mathbf{2 a}(0.4 \mathrm{mmol})$ in $4.0 \mathrm{~mL}$ of solvent. After $\mathbf{1}$ or $\mathbf{2 a}$ was consumed, $\mathrm{Et}_{3} \mathrm{~N}$ was added to quench the reaction. ${ }^{b}$ Isolated yield. ${ }^{c}$ Determined by HPLC using a chiral stationary. 


\section{Table S3 Effect of Ligand ${ }^{\mathrm{a}}$.}

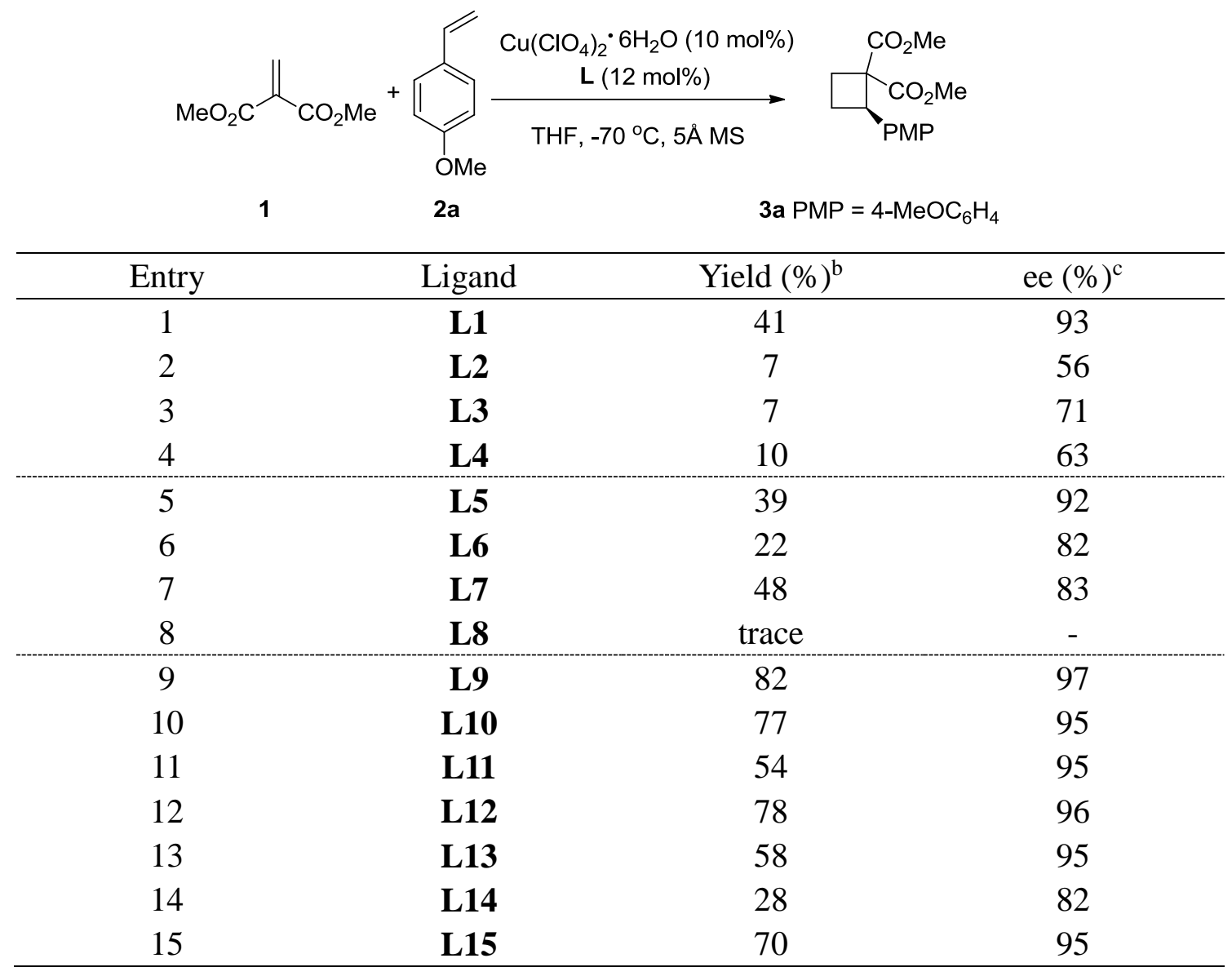

${ }^{a}$ Reaction conditions: $\mathrm{Cu}\left(\mathrm{ClO}_{4}\right)_{2} \cdot 6 \mathrm{H}_{2} \mathrm{O}(0.04 \mathrm{mmol}), \mathbf{L}(0.048 \mathrm{mmol}), 5 \AA \mathrm{MS}(100 \mathrm{mg}), \mathbf{1}(1.0$ mmol), and $\mathbf{2 a}(0.4 \mathrm{mmol})$ in $4.0 \mathrm{~mL}$ of THF. After $\mathbf{1}$ or $\mathbf{2 a}$ was consumed, $\mathrm{Et}_{3} \mathrm{~N}$ was added to quench the reaction. ${ }^{b}$ Isolated yield. ${ }^{c}$ Determined by HPLC using a chiral stationary. 


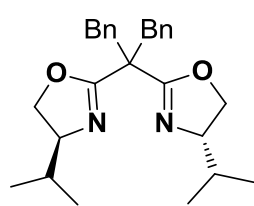

L1

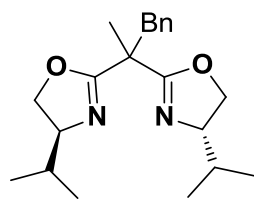

L5

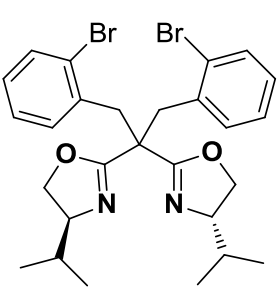

L9

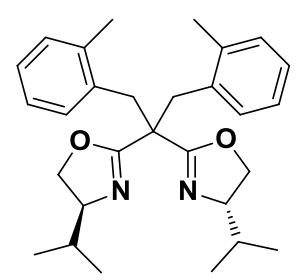

L13

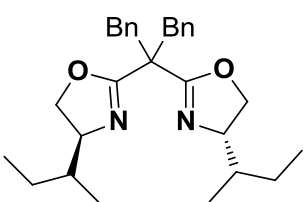

L3

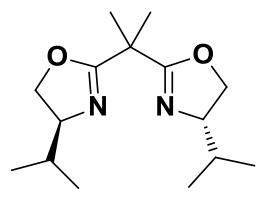

L6

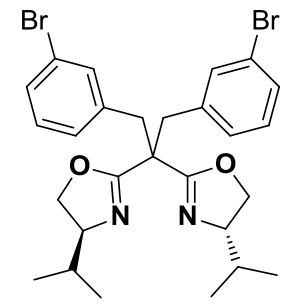

L10

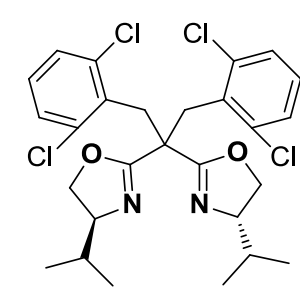

L14

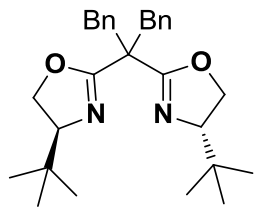

L2

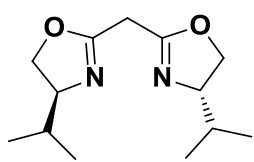

L7

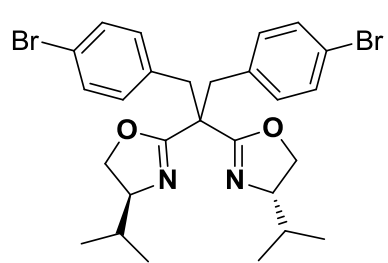

L11

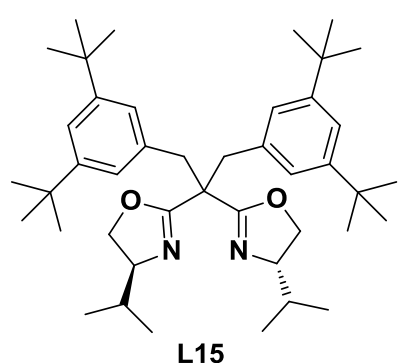

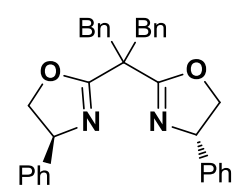

L4

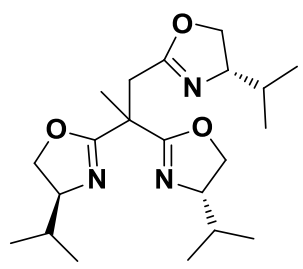

L8

Figure S1 Ligands Screened 


\section{Scheme S1. Reactions for $\beta$-Substituted Methylidenemalonate}

$$
\begin{aligned}
& \begin{array}{c}
\mathrm{MeO}_{2} \mathrm{C} \\
\text { dimethyl 2-ethylidenemalonate }
\end{array}
\end{aligned}
$$

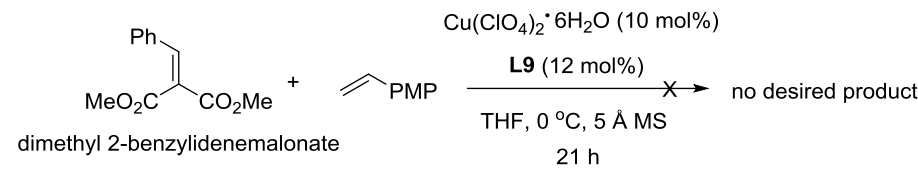

\section{Scheme S2. Substrate Scope Extension}
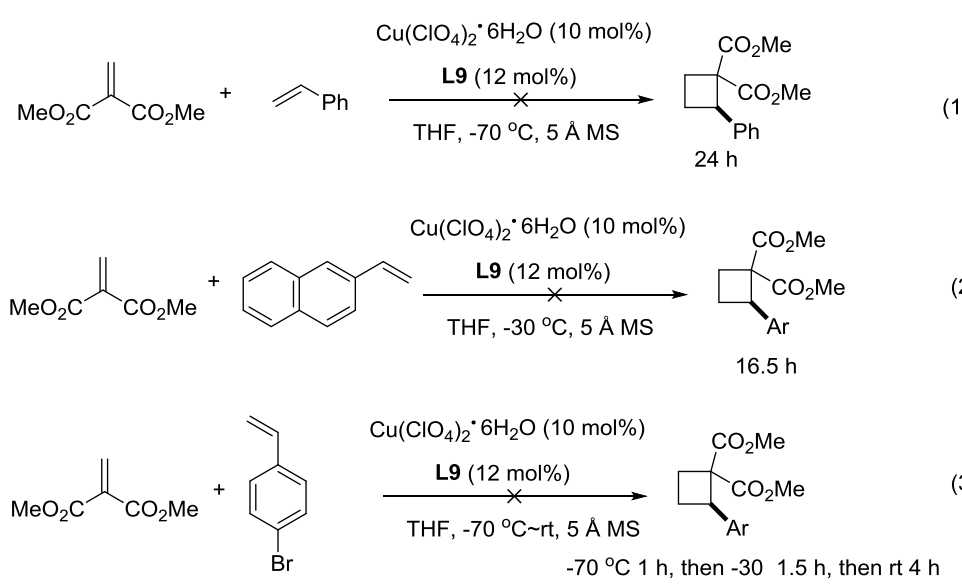
$\mathrm{Cu}\left(\mathrm{ClO}_{4}\right)_{2} \cdot 6 \mathrm{H}_{2} \mathrm{O}(10 \mathrm{~mol} \%)$
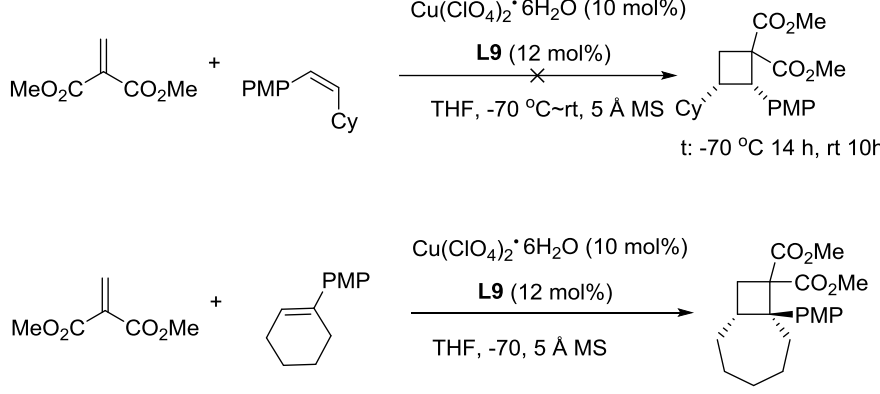

yield: $66 \%$, ee: $84 \%$ dr: $>99 / 1$
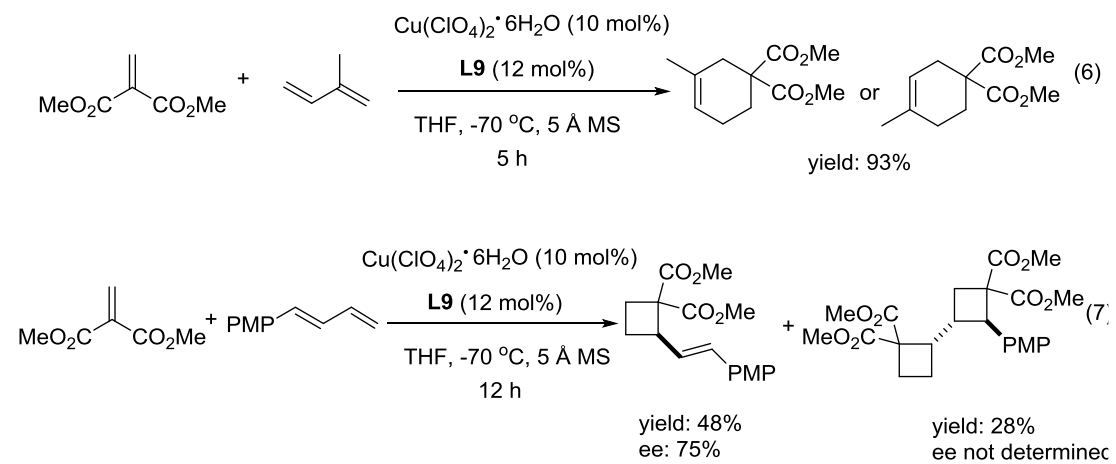
Scheme S3. Molecule Sieves (MS) effect

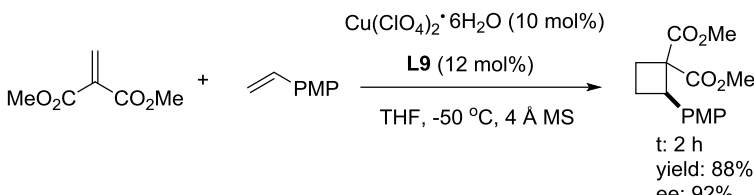

$$
\begin{aligned}
& \mathrm{Cu}\left(\mathrm{ClO}_{4}\right)_{2} \cdot 6 \mathrm{H}_{2} \mathrm{O}(10 \mathrm{~mol} \%) \quad \mathrm{CO}_{2} \mathrm{Me}
\end{aligned}
$$

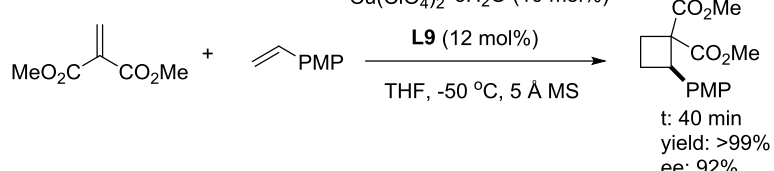

\section{Scheme S4. Control Experiments}

$$
\begin{aligned}
& \mathrm{MeO}_{2} \mathrm{C}{ }_{\mathrm{CO}_{2} \mathrm{Me}}^{+} ح_{\mathrm{PMP}} \frac{\text { no catalyst }}{\underset{\mathrm{THF},-78{ }^{\circ} \mathrm{C}, 5 \mathrm{AMS}}{\longrightarrow}} \underset{\substack{\mathrm{PMP} \\
\mathrm{CO}_{2} \mathrm{Me}}}{\mathrm{CO}_{2} \mathrm{Me}}
\end{aligned}
$$

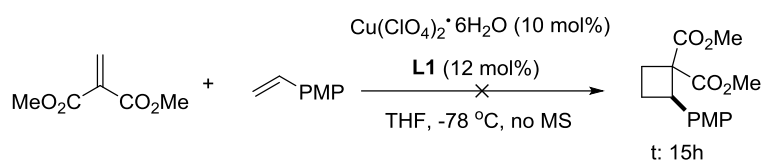

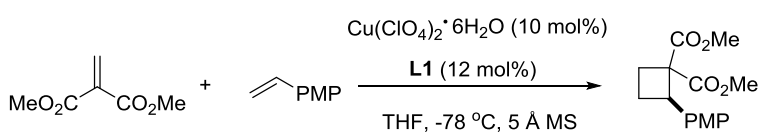

$$
\begin{aligned}
& \mathrm{t}: 9 \mathrm{~h} \\
& \text { yield: } 60 \% \\
& \text { ee: } 97 \%
\end{aligned}
$$




\section{General Experimental Procedure}

Standard Procedure: a mixture of $\mathrm{Cu}\left(\mathrm{ClO}_{6}\right)_{2} \cdot 6 \mathrm{H}_{2} \mathrm{O}(0.02 \mathrm{mmol})$ and ligand (L9, $0.024 \mathrm{mmol})$ in THF ( $3 \mathrm{~mL})$ with activated $5 \AA$ MS was stirred at room temperature for $1.5 \mathrm{~h}$ under atmosphere of nitrogen. Then the mixture was cooled to $-70{ }^{\circ} \mathrm{C}$ for 30 minutes, and dimethyl methylene malonate $(1.0 \mathrm{mmol})$ was mixed with 4-methoxy styrene $(0.40 \mathrm{mmol})$ in $1 \mathrm{~mL}$ of $\mathrm{THF}$, cooled to $-70{ }^{\circ} \mathrm{C}$, and added to the reaction mixture while cold. The resulting solution was stirred until the styrene was completely consumed (monitored by TLC, hexane/ethyl acetate $=5 / 1$ ). Then the mixture was quenched with $\mathrm{Et}_{3} \mathrm{~N}$ at $-70{ }^{\circ} \mathrm{C}$, then passed through a short silica gel column and eluted with DCM (50 mL). The combined elution was concentrated under reduced pressure to give the crude product for dr determination, which was further purified by flash chromatography on silica gel (For $\mathbf{3 m}, \mathrm{Al}_{2} \mathrm{O}_{3}$ column was used for purification) (hexane/ethyl acetate $=20 / 1$ ).

3e-3f: a mixture of $\mathrm{Cu}(\mathrm{OTf})_{2}(0.04 \mathrm{mmol})$ and ligand $(\mathbf{L 1}, 0.048 \mathrm{mmol})$ in THF (1 $\mathrm{mL}$ ) with activated $5 \AA \mathrm{MS}$ was stirred at room temperature for $1.5 \mathrm{~h}$ under atmosphere of nitrogen. Then the mixture was cooled to $-80{ }^{\circ} \mathrm{C}$ for 30 minutes, and dimethyl methylene malonate $(1.0 \mathrm{mmol})$ was mixed with 4-methoxy styrene $(0.40$ mmol) in $1 \mathrm{~mL}$ of $\mathrm{THF}$, cooled to $-80^{\circ} \mathrm{C}$, and added to the reaction mixture while cold The resulting solution was stirred until the styrene was completely consumed (monitored by TLC, hexane/ethyl acetate $=5 / 1$ ). Then the mixture was quenched with $\mathrm{Et}_{3} \mathrm{~N}$ at $-80{ }^{\circ} \mathrm{C}$, then passed through a short silica gel column and eluted with DCM $(50 \mathrm{~mL})$. The combined elution was concentrated under reduced pressure to give the crude product, which was further purified by flash chromatography on silica gel (hexane/ethyl acetate $=20 / 1$ ). 


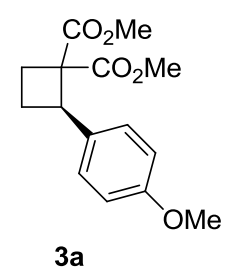

(R)-dimethyl 2-(4-methoxyphenyl)cyclobutane-1,1-dicarboxylate

Colorless liquid 1.5 h, 91.8 mg, (82\%); 97\% ee, determined by HPLC analysis: Daicel Chiralcel AD-H column $(25 \mathrm{~cm})$, hexanes $/{ }^{i} \mathrm{PrOH}=98 / 2,0.6 \mathrm{~mL} / \mathrm{min}, 240 \mathrm{~nm}$; $\mathrm{t}_{\mathrm{r}}($ major $)=19.7 \mathrm{~min}, \mathrm{t}_{\mathrm{r}}($ minor $)=17.7 \mathrm{~min} ;[\alpha]_{\mathrm{D}}{ }^{30.0}=+91.1^{\circ}\left(\mathrm{c}=0.81, \mathrm{CHCl}_{3}\right) ;{ }^{1} \mathrm{H}$ NMR (400 MHz, $\left.\mathrm{CDCl}_{3}\right): \delta 7.21(\mathrm{~d}, \mathrm{~J}=8.8 \mathrm{~Hz}, 2 \mathrm{H}), 6.82(\mathrm{~d}, J=8.8 \mathrm{~Hz}, 2 \mathrm{H}), 4.31$ $(\mathrm{t}, J=9.6 \mathrm{~Hz}, 1 \mathrm{H}), 3.78(\mathrm{~s}, 3 \mathrm{H}), 3.77(\mathrm{~s}, 3 \mathrm{H}), 3.28(\mathrm{~s}, 3 \mathrm{H}), 2.71-2.52(\mathrm{~m}, 2 \mathrm{H})$, 2.28-2.11 (m, 2H); ${ }^{13} \mathrm{C}$ NMR (100 $\left.\mathrm{MHz}, \mathrm{CDCl}_{3}\right): \delta$ 172.1, 169.8, 158.5, 131.1, 128.6, 113.3, 59.7, 55.1, 52.4, 51.9, 44.5, 25.4, 20.9; IR (neat, $\mathrm{cm}^{-1}$ ): 2953, 2838, 1727, 1612, 1513, 1434, 1249, 1199, 1180, 1103, 1034, 833, 804, 702; HRMS-ESI: $[\mathrm{M}+\mathrm{Na}]^{+}$Calculated for $\mathrm{C}_{15} \mathrm{H}_{18} \mathrm{NaO}_{5}{ }^{+}, 301.1046$; Found: 301.1047 .

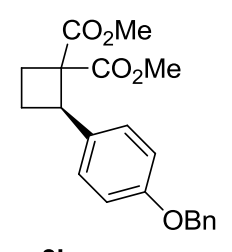

$3 \mathbf{b}$

(R)-dimethyl 2-(4-(benzyloxy)phenyl)cyclobutane-1,1-dicarboxylate

Colorless liquid 4 h, 129.4 mg, (91\%); 96\% ee, determined by HPLC analysis: Daicel Chiralcel AD-H column $(25 \mathrm{~cm})$, hexanes $/{ }^{i} \mathrm{PrOH}=95 / 5,0.7 \mathrm{~mL} / \mathrm{min}, 240 \mathrm{~nm}$; $\mathrm{t}_{\mathrm{r}}($ major $)=17.0 \mathrm{~min}, \mathrm{t}_{\mathrm{r}}(\operatorname{minor})=14.1 \mathrm{~min} ;[\alpha]_{\mathrm{D}}{ }^{28.5}=+52.2^{\circ}\left(\mathrm{c}=1.66, \mathrm{CHCl}_{3}\right) ;{ }^{1} \mathrm{H}$ NMR (400 MHz, $\left.\mathrm{CDCl}_{3}\right): \delta$ 7.42-7.30 (m, 5H), $7.21(\mathrm{~d}, J=8.4 \mathrm{~Hz}, 2 \mathrm{H}), 6.90$ (d, $J=$ $8.4 \mathrm{~Hz}, 2 \mathrm{H}), 5.04(\mathrm{~s}, 2 \mathrm{H}), 4.31(\mathrm{t}, J=9.4 \mathrm{~Hz}, 1 \mathrm{H}), 3.76(\mathrm{~s}, 3 \mathrm{H}), 3.24(\mathrm{~s}, 3 \mathrm{H})$, 2.71-2.54 (m, 2H), 2.28-2.13 (m, 2H); $\left.{ }^{13} \mathrm{C} \mathrm{NMR} \mathrm{(100} \mathrm{MHz,} \mathrm{CDCl}_{3}\right): \delta$ 172.2, 169.8, $157.7,137.0,131.4,128.6,128.5,127.9,127.4,114.4,69.9,59.7,52.5,51.9$, 44.6, 25.5, 20.9; IR (neat, $\mathrm{cm}^{-1}$ ): 2946, 1725, 1609, 1514, 1454, 1433, 1250, 1189, 1105, 1040, 841, 815, 739, 696; HRMS-ESI: $\left[\mathrm{M}+\mathrm{NH}_{4}\right]^{+}$Calculated for $\mathrm{C}_{21} \mathrm{H}_{26} \mathrm{NO}_{5}{ }^{+}$, 372.1805; Found: 372.1807. 


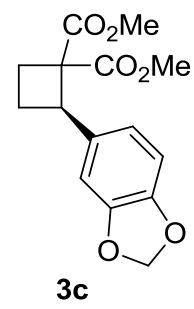

(R)-dimethyl-2-(benzo[d][1,3]dioxol-5-yl)cyclobutane-1,1-dicarboxylate

Colorless liquid 3 h, 70.0 mg, (60\%); 96\% ee, determined by HPLC analysis: Daicel Chiralcel ID column $(25 \mathrm{~cm})$, hexanes $/{ }^{i} \mathrm{PrOH}=95 / 5,0.7 \mathrm{~mL} / \mathrm{min}, 254 \mathrm{~nm} ; \mathrm{t}$ $($ major $)=14.1 \mathrm{~min}, \mathrm{t}($ minor $)=20.0 \mathrm{~min} ;[\alpha]_{\mathrm{D}}^{28.6}=+78.1^{\circ}\left(\mathrm{c}=1.59, \mathrm{CHCl}_{3}\right) ;{ }^{1} \mathrm{H}$ $\operatorname{NMR}\left(400 \mathrm{MHz}, \mathrm{CDCl}_{3}\right): \delta 6.82(\mathrm{~s}, 1 \mathrm{H}), 6.74(\mathrm{~s}, 2 \mathrm{H}), 5.91(\mathrm{~s}, 2 \mathrm{H}), 4.26(\mathrm{t}, J=9.6 \mathrm{~Hz}$, 1H), 3.77 (s, 3H), 3.35 (s, 3H), 2.69-2.48 (m, 2H), 2.27-2.10 (m, 2H); ${ }^{13} \mathrm{C}$ NMR (100 $\left.\mathrm{MHz}, \mathrm{CDCl}_{3}\right): \delta 172.0,169.7,147.3,146.4,133.0,120.5,108.2,107.7,100.8,59.7$, 52.5, 52.0, 44.9, 25.5, 21.1; IR (neat, $\mathrm{cm}^{-1}$ ): 2998, 2953, 2898, 1726, 1487, 1435, 1332, 1254, 1198, 1099, 1036, 932, 874, 810, 706, 627; HRMS-ESI: $\left[\mathrm{M}+\mathrm{NH}_{4}\right]^{+}$ Calculated for $\mathrm{C}_{15} \mathrm{H}_{20} \mathrm{NO}_{6}{ }^{+}, 310.1285$; Found: 310.1288 .

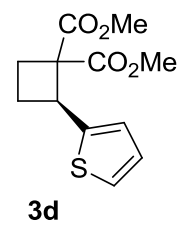

(S)-dimethyl 2-(thiophen-2-yl)cyclobutane-1,1-dicarboxylate

White paste $4.5 \mathrm{~h}, 25.6 \mathrm{mg}$, (25\%); 95\% ee, determined by HPLC analysis: Daicel Chiralcel OD-H column $(25 \mathrm{~cm})$, hexanes $/{ }^{i} \mathrm{PrOH}=95 / 5,0.7 \mathrm{~mL} / \mathrm{min}, 250 \mathrm{~nm}$; $\mathrm{t}_{\mathrm{r}}($ major $)=10.4 \mathrm{~min}, \mathrm{t}_{\mathrm{r}}(\operatorname{minor})=12.6 \mathrm{~min} ;[\alpha]_{\mathrm{D}}{ }^{28.4}=+47.5^{\circ}\left(\mathrm{c}=1.16, \mathrm{CHCl}_{3}\right) ;{ }^{1} \mathrm{H}$ NMR (400 MHz, $\left.\mathrm{CDCl}_{3}\right): \delta 7.18-7.17(\mathrm{~m}, 1 \mathrm{H}), 6.95-6.91(\mathrm{~m}, 2 \mathrm{H}), 4.50(\mathrm{t}, J=9.6 \mathrm{~Hz}$, $1 \mathrm{H}), 3.78(\mathrm{~s}, 3 \mathrm{H}), 3.37(\mathrm{~s}, 3 \mathrm{H}), 2.75-2.69(\mathrm{~m}, 1 \mathrm{H}), 2.59-2.49(\mathrm{~m}, 1 \mathrm{H}), 2.36-2.20(\mathrm{~m}$, $2 \mathrm{H}) ;{ }^{13} \mathrm{C} \mathrm{NMR}\left(100 \mathrm{MHz}, \mathrm{CDCl}_{3}\right): \delta 171.7,169.4,142.6,126.7,125.0,124.5,60.1$, 52.6, 52.2, 40.6, 25.5, 23.8; IR (neat, $\mathrm{cm}^{-1}$ ): 3001, 2953, 1724, 1433, 1261, 1201, 1104, 1030, 956, 860, 798, 704; HRMS-ESI: $\left[\mathrm{M}+\mathrm{NH}_{4}\right]^{+}$Calculated for $\mathrm{C}_{12} \mathrm{H}_{18} \mathrm{NO}_{4} \mathrm{~S}^{+}$, 272.0951; Found: 272.0953. 


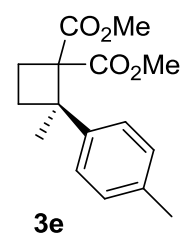

(R)-dimethyl 2-methyl-2-(4-methylphenyl)cyclobutane-1,1-dicarboxylate

Colorless liquid 6 h, $57.8 \mathrm{mg}$, (52\%); 94\% ee, determined by HPLC analysis: Daicel Chiralcel AD-H column $(25 \mathrm{~cm})$, hexanes $/{ }^{i} \mathrm{PrOH}=98 / 2,0.6 \mathrm{~mL} / \mathrm{min}, 270 \mathrm{~nm}$; $\mathrm{t}($ major $)=11.2 \mathrm{~min}, \mathrm{t}($ minor $)=9.7 \mathrm{~min} ;[\alpha]_{\mathrm{D}}^{28.8}=+61.9^{\circ}\left(\mathrm{c}=0.80, \mathrm{CHCl}_{3}\right) ;{ }^{1} \mathrm{H}$ NMR (400 MHz, $\left.\mathrm{CDCl}_{3}\right): \delta 7.21(\mathrm{~d}, J=8.0 \mathrm{~Hz}, 2 \mathrm{H}), 7.08(\mathrm{~d}, J=8.0 \mathrm{~Hz}, 2 \mathrm{H}), 3.84(\mathrm{~s}$, $3 \mathrm{H}), 3.35(\mathrm{~s}, 3 \mathrm{H}), 2.98-2.91(\mathrm{~m}, 1 \mathrm{H}), 2.68-2.60(\mathrm{~m}, 1 \mathrm{H}), 2.42-2.36(\mathrm{~m}, 1 \mathrm{H}), 2.30(\mathrm{~s}$, $3 \mathrm{H}), 1.90-1.84(\mathrm{~m}, 1 \mathrm{H}), 1.51(\mathrm{~s}, 3 \mathrm{H}) ;{ }^{13} \mathrm{C} \mathrm{NMR}\left(100 \mathrm{MHz}, \mathrm{CDCl}_{3}\right): \delta 171.3,170.9$, 144.0, 135.4, 128.7, 125.1, 61.7, 52.1, 52.0, 50.3, 29.1, 28.9, 24.5, 21.0; IR (neat, $\left.\mathrm{cm}^{-1}\right): 2952,1730,1516,1433,1255,1228,1134,1106,1076,1022,818,724$ HRMS-EI: $\left[\mathrm{M}+\mathrm{NH}_{4}\right]^{+}$Calculated for $\mathrm{C}_{16} \mathrm{H}_{24} \mathrm{NO}_{4}{ }^{+}$, 294.1700; Found: 294.1704.

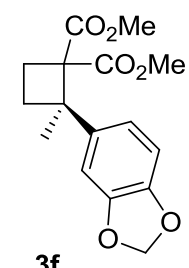

(R)-dimethyl-2-methyl-2-(benzo[d][1,3]dioxol-5-yl)cyclobutane-1,1-dicarboxylate

Colorless liquid 8 h, $88.3 \mathrm{mg}$, (72\%); 96\% ee, determined by HPLC analysis: Daicel Chiralcel AD-H column $(25 \mathrm{~cm})$, hexanes $/{ }^{i} \mathrm{PrOH}=98 / 2,0.6 \mathrm{~mL} / \mathrm{min}, 230 \mathrm{~nm}$; $\mathrm{t}($ major $)=18.3 \min , \mathrm{t}($ minor $)=17.4 \mathrm{~min} ;[\alpha]_{\mathrm{D}}^{28.7}=+53.4^{\circ}\left(\mathrm{c}=1.23, \mathrm{CHCl}_{3}\right) ;{ }^{1} \mathrm{H}$ NMR (400 MHz, $\left.\mathrm{CDCl}_{3}\right): \delta 6.88(\mathrm{~s}, 1 \mathrm{H}), 6.78-6.71(\mathrm{~m}, 2 \mathrm{H}), 5.91(\mathrm{~s}, 2 \mathrm{H}), 3.84(\mathrm{~s}, 3 \mathrm{H})$, $3.43(\mathrm{~s}, 3 \mathrm{H}), 2.93-2.86(\mathrm{~m}, 1 \mathrm{H}), 2.66-2.58(\mathrm{~m}, 1 \mathrm{H}), 2.40-2.35(\mathrm{~m}, 1 \mathrm{H}), 1.88-1.82(\mathrm{~m}$, 1H), 1.49 (s, 3H); $\left.{ }^{13} \mathrm{C} \mathrm{NMR} \mathrm{(100} \mathrm{MHz,} \mathrm{CDCl}_{3}\right): \delta 171.1,170.8,147.3,145.6,141.2$, 118.2, 107.8, 106.2, 100.7, 61.6, 52.1, 50.3, 29.2, 24.5; IR (neat, $\mathrm{cm}^{-1}$ ): 2959, 1732, 1489, 1435, 1261, 1227, 1091, 1038, 934, 808, 641; HRMS-EI: $\left[\mathrm{M}+\mathrm{NH}_{4}\right]^{+}$ Calculated for $\mathrm{C}_{16} \mathrm{H}_{22} \mathrm{NO}_{6}{ }^{+}, 324.1442$; Found: 324.1444 . 


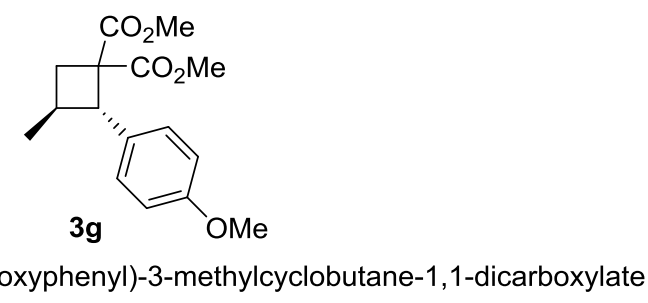

Colorless liquid 12 h, 104.3 mg, (89\%, >95/5 dr); 97\% ee, determined by HPLC analysis: Daicel Chiralcel AD-H column $(25 \mathrm{~cm})$, hexanes $/{ }^{i} \mathrm{PrOH}=98 / 2,0.6 \mathrm{~mL} / \mathrm{min}$, $280 \mathrm{~nm} ; \mathrm{t}_{\mathrm{r}}($ major $)=12.6 \mathrm{~min}, \mathrm{t}_{\mathrm{r}}($ minor $)=14.6 \mathrm{~min} ;[\alpha]_{\mathrm{D}}^{29.9}=-24.8^{\circ}(\mathrm{c}=0.89$, $\left.\mathrm{CHCl}_{3}\right) ;{ }^{1} \mathrm{H} \mathrm{NMR}\left(400 \mathrm{MHz}, \mathrm{CDCl}_{3}\right): \delta 7.20(\mathrm{~d}, J=8.8 \mathrm{~Hz}, 2 \mathrm{H}), 6.83(\mathrm{~d}, J=8.4 \mathrm{~Hz}$, 2H), 3.80-3.76 (m, 7H), 3.28 (s, 3H), 2.98-2.89 (m, 1H), 2.84-2.80 (m, 1H), 1.82-1.77 $(\mathrm{m}, 1 \mathrm{H}), 1.14(\mathrm{~d}, J=6.4 \mathrm{~Hz}, 3 \mathrm{H}) ;{ }^{13} \mathrm{C} \mathrm{NMR}\left(100 \mathrm{MHz}, \mathrm{CDCl}_{3}\right): \delta 172.1,169.9,158.5$, 130.3, 128.6, 113.4, 57.0, 55.1, 52.4, 52.3, 51.9, 33.6, 29.1, 20.3; IR (neat, $\mathrm{cm}^{-1}$ ): 2953, 2839, 1727, 1612, 1513, 1435, 1180, 1116, 1066, 1034, 926, 838, 794; HRMS-ESI: $\left[\mathrm{M}+\mathrm{NH}_{4}\right]^{+}$Calculated for $\mathrm{C}_{16} \mathrm{H}_{24} \mathrm{NO}_{5}{ }^{+}, 310.1649$; Found: 310.1650 .

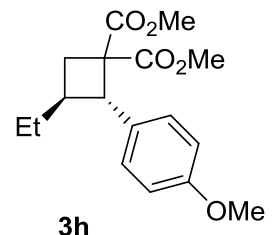

(2S,3S)-dimethyl 3-ethyl-2-(4-methoxyphenyl)cyclobutane-1,1-dicarboxylate

Colorless liquid 3h, $103.2 \mathrm{mg}$, (84\%, >95/5 dr); 98\% ee, determined by HPLC analysis: Daicel Chiralcel AD-H column $(25 \mathrm{~cm})$, hexanes $/{ }^{i} \mathrm{PrOH}=99 / 1,0.6 \mathrm{~mL} / \mathrm{min}$, $280 \mathrm{~nm} ; \mathrm{t}_{\mathrm{r}}($ major $)=16.6 \mathrm{~min}, \mathrm{t}_{\mathrm{r}}($ minor $)=21.6 \mathrm{~min} ;[\alpha]_{\mathrm{D}}^{28.8}=-37.2^{\circ}(\mathrm{c}=1.25$, $\left.\mathrm{CHCl}_{3}\right) ;{ }^{1} \mathrm{H} \mathrm{NMR}\left(400 \mathrm{MHz}, \mathrm{CDCl}_{3}\right): \delta 7.21(\mathrm{~d}, J=8.4 \mathrm{~Hz}, 2 \mathrm{H}), 6.82(\mathrm{~d}, J=8.8 \mathrm{~Hz}$, 2H), 3.86-3.83 (m, 1H), $3.78(\mathrm{~s}, 3 \mathrm{H}), 3.75(\mathrm{~s}, 3 \mathrm{H}), 3.27(\mathrm{~s}, 3 \mathrm{H}), 2.84-2.78(\mathrm{~m}, 2 \mathrm{H})$, 1.82-1.74 (m, 1H), 1.58-1.42 (m, 2H), $0.82(\mathrm{t}, J=7.6 \mathrm{~Hz}, 3 \mathrm{H}) ;{ }^{13} \mathrm{C}$ NMR $(100 \mathrm{MHz}$, $\left.\mathrm{CDCl}_{3}\right): \delta 172.2,170.0,158.5,130.8,128.7,113.4,56.7,55.1,52.4,52.0,50.9,35.6$, 31.7, 28.9, 11.2; IR (neat, $\mathrm{cm}^{-1}$ ): 2954, 1728, 1612, 1513, 1434, 1247, 1180, 1126, 1035, 841, 793, 706; HRMS-ESI: $\left[\mathrm{M}+\mathrm{NH}_{4}\right]^{+}$Calculated for $\mathrm{C}_{17} \mathrm{H}_{26} \mathrm{NO}_{5}^{+}, 324.1805$; Found: 324.1807 


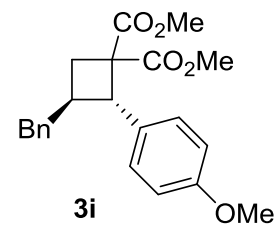

(2S,3S)-dimethyl 3-benzyl-2-(4-methoxyphenyl)cyclobutane-1,1-dicarboxylate

Colorless liquid 55 h, $127.5 \mathrm{mg}$, (87\%, >99/1 dr); >99\% ee, determined by HPLC analysis: Daicel Chiralcel AD-H column $(25 \mathrm{~cm})$, hexanes $/{ }^{i} \mathrm{PrOH}=98 / 2,0.6$ $\mathrm{mL} / \mathrm{min}, 230 \mathrm{~nm} ; \mathrm{t}_{\mathrm{r}}($ major $)=18.6 \mathrm{~min}, \mathrm{t}_{\mathrm{r}}($ minor $)=9.7 \mathrm{~min} ;[\alpha]_{\mathrm{D}}^{28.3}=-40.8^{\circ}(\mathrm{c}=$ 1.45, $\left.\mathrm{CHCl}_{3}\right) ;{ }^{1} \mathrm{H} \mathrm{NMR}\left(400 \mathrm{MHz}, \mathrm{CDCl}_{3}\right): \delta$ 7.26-7.23 (m, 2H), 7.19-7.12 (m, 5H), $6.79(\mathrm{~d}, J=8.8 \mathrm{~Hz}, 2 \mathrm{H}), 3.97(\mathrm{~d}, J=10.4 \mathrm{~Hz}, 1 \mathrm{H}), 3.77$ (s, 3H), 3.76 (s, 3H), 3.27 (s, $3 \mathrm{H}), 3.17-3.07(\mathrm{~m}, 1 \mathrm{H}), 2.91-2.86(\mathrm{~m}, 1 \mathrm{H}), 2.78-2.64(\mathrm{~m}, 2 \mathrm{H}), 1.95-1.90(\mathrm{~m}, 1 \mathrm{H}),{ }^{13} \mathrm{C}$ NMR $\left(100 \mathrm{MHz}, \mathrm{CDCl}_{3}\right): \delta 172.1,169.8,158.6,139.4,130.0,128.7,128.6,128.4$, 126.1, 113.4, 56.9, 55.2, 52.5, 52.0, 50.7, 41.6, 35.4, 32.0; IR (neat, $\mathrm{cm}^{-1}$ ): 2951, 2838, 1727, 1612, 1513, 1434, 1247, 1201, 1178, 1127, 1032, 838, 743, 700; HRMS-ESI: $\left[\mathrm{M}+\mathrm{NH}_{4}\right]^{+}$Calculated for $\mathrm{C}_{22} \mathrm{H}_{28} \mathrm{NO}_{5}{ }^{+}, 386.1962$; Found: 386.1964 .

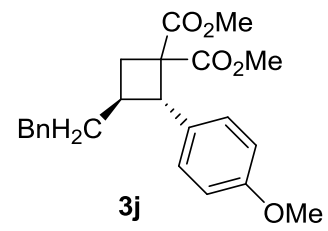

(2S,3S)-dimethyl 2-(4-methoxyphenyl)-3-phenethylcyclobutane-1,1-dicarboxylate

Colorless liquid 10 h, $139.8 \mathrm{mg},(91 \%,>95 / 5 \mathrm{dr}) ; 97 \%$ ee, determined by HPLC analysis: Daicel Chiralcel IA-3 column $(25 \mathrm{~cm})$, hexanes $/{ }^{i} \mathrm{PrOH}=95 / 5,0.7 \mathrm{~mL} / \mathrm{min}$, $240 \mathrm{~nm} ; \mathrm{t}_{\mathrm{r}}($ major $)=9.2 \mathrm{~min}, \mathrm{t}_{\mathrm{r}}($ minor $)=11.6 \mathrm{~min} ;[\alpha]_{\mathrm{D}}^{28.8}=-15.6^{\circ}(\mathrm{c}=1.42$, $\left.\mathrm{CHCl}_{3}\right) ;{ }^{1} \mathrm{H} \mathrm{NMR}\left(400 \mathrm{MHz}, \mathrm{CDCl}_{3}\right): \delta 7.25-7.15(\mathrm{~m}, 5 \mathrm{H}), 7.09(\mathrm{~d}, J=7.2 \mathrm{~Hz}, 2 \mathrm{H})$, $6.81(\mathrm{~d}, J=8.8 \mathrm{~Hz}, 2 \mathrm{H})), 3.90(\mathrm{~d}, J=9.6 \mathrm{~Hz}, 1 \mathrm{H}), 3.77(\mathrm{~s}, 3 \mathrm{H}), 3.74(\mathrm{~s}, 3 \mathrm{H}), 3.26(\mathrm{~s}$, 3H), 2.92-2.79 (m, 2H), 2.54-2.50 (m, 2H), 1.87-1.72 (m, 3H); ${ }^{13} \mathrm{C}$ NMR (100 MHz, $\left.\mathrm{CDCl}_{3}\right): \delta 172.1,169.9,158.6,141.8,130.4,128.8,128.2(1), 128.1(8), 125.7,113.4$, 56.9, 55.1, 52.4, 51.9, 51.1, 37.9, 33.8, 33.2, 32.0; IR (neat, $\mathrm{cm}^{-1}$ ): 2950, 2848, 1728, 1612, 1512, 1434, 1247, 1179, 1128, 1032, 840, 749, 699; HRMS-ESI: $\left[\mathrm{M}+\mathrm{NH}_{4}\right]^{+}$ Calculated for $\mathrm{C}_{23} \mathrm{H}_{30} \mathrm{NO}_{5}{ }^{+}$, 400.2118; Found: 400.2123 . 


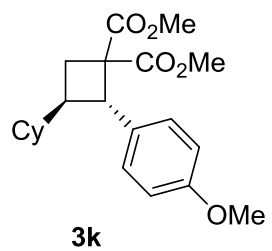

(2S,3R)-dimethyl 3-cyclohexyl-2-(4-methoxyphenyl)cyclobutane-1,1-dicarboxylate

Colorless liquid 24 h, 72.0 mg, (50\%, 93/7 dr); 99\% ee, determined by HPLC analysis: Daicel Chiralcel AD-H column $(25 \mathrm{~cm})$, hexanes $/{ }^{i} \mathrm{PrOH}=98 / 2,0.6 \mathrm{~mL} / \mathrm{min}$, $240 \mathrm{~nm} ; \mathrm{t}_{\mathrm{r}}($ major $)=12.8 \mathrm{~min}, \mathrm{t}_{\mathrm{r}}($ minor $)=18.5 \mathrm{~min} ;[\alpha]_{\mathrm{D}}^{28.7}=-24.9^{\circ}(\mathrm{c}=1.49$, $\left.\mathrm{CHCl}_{3}\right) ;{ }^{1} \mathrm{H}$ NMR $\left(400 \mathrm{MHz}, \mathrm{CDCl}_{3}\right): \delta 7.21(\mathrm{~d}, J=8.8 \mathrm{~Hz}, 2 \mathrm{H}), 6.81(\mathrm{~d}, J=8.8 \mathrm{~Hz}$, 2H), $3.92(\mathrm{~d}, J=10.0 \mathrm{~Hz}, 1 \mathrm{H}), 3.77(\mathrm{~s}, 3 \mathrm{H}), 3.74(\mathrm{~s}, 3 \mathrm{H}), 3.24(\mathrm{~s}, 3 \mathrm{H}), 2.79-2.74(\mathrm{~m}$, $1 \mathrm{H}), 2.63-2.54(\mathrm{~m}, 1 \mathrm{H}), 1.83-1.78(\mathrm{~m}, 1 \mathrm{H}), 1.71-1.68(\mathrm{~m}, 2 \mathrm{H}), 1.61-1.55(\mathrm{~m}, 2 \mathrm{H})$, $1.48-1.45(\mathrm{~m}, 1 \mathrm{H}), 1.31-1.06(\mathrm{~m}, 4 \mathrm{H}), 0.91-0.82(\mathrm{~m}, 1 \mathrm{H}), 0.75-0.65(\mathrm{~m}, 1 \mathrm{H}) ;{ }^{13} \mathrm{C}$ NMR (100 MHz, $\left.\mathrm{CDCl}_{3}\right): \delta$ 172.2, 170.0, 158.4, 131.3, 128.8, 113.3, 56.5, 55.1, 52.4, 51.9, 50.0, 44.9, 39.3, 30.5, 30.4, 29.9, 26.3, 25.9(1), 25.8(7); IR (neat, $\mathrm{cm}^{-1}$ ): 2922, 2849, 1729, 1612, 1512, 1435, 1301, 1245, 1179, 1131, 1036, 840, 708; HRMS-ESI: $\left[\mathrm{M}+\mathrm{NH}_{4}\right]^{+}$Calculated for $\mathrm{C}_{21} \mathrm{H}_{32} \mathrm{NO}_{5}{ }^{+}, 378.2275$; Found: 378.2276 .

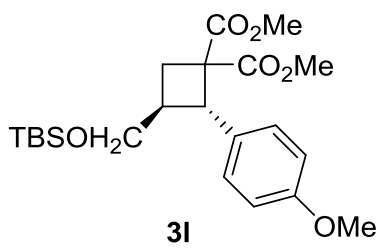

(2S,3S)-dimethyl 3-((tert-butyldimethylsilyl)oxy)methyl)-2-(4methoxyphenyl)cyclobutane-1,1-dicarboxylate

Colorless liquid $40 \mathrm{~min}, 154.1 \mathrm{mg},(91 \%$, >99/1 dr); $99 \%$ ee, determined by HPLC analysis: Daicel Chiralcel IC-3 column $(25 \mathrm{~cm})$, hexanes $/{ }^{i} \mathrm{PrOH}=90 / 10,0.7$ $\mathrm{mL} / \mathrm{min}, 280 \mathrm{~nm} ; \mathrm{t}_{\mathrm{r}}($ major $)=7.8 \mathrm{~min}, \mathrm{t}_{\mathrm{r}}($ minor $)=6.9 \mathrm{~min} ;[\alpha]_{\mathrm{D}}^{30.0}=-7.5^{\circ}(\mathrm{c}=1.52$, $\left.\mathrm{CHCl}_{3}\right) ;{ }^{1} \mathrm{H} \mathrm{NMR}\left(400 \mathrm{MHz}, \mathrm{CDCl}_{3}\right): \delta 7.22(\mathrm{~d}, J=8.8 \mathrm{~Hz}, 2 \mathrm{H}), 6.81(\mathrm{~d}, J=8.4 \mathrm{~Hz}$, 2H), $4.13(\mathrm{~d}, J=10.0 \mathrm{~Hz}, 1 \mathrm{H}), 3.77(\mathrm{~s}, 3 \mathrm{H}), 3.75(\mathrm{~s}, 3 \mathrm{H}), 3.63-3.55(\mathrm{~m}, 2 \mathrm{H}), 3.28(\mathrm{~s}$, 3H), 3.07-2.98 (m, 1H), 2.69-2.64 (m, 1H), 2.14-2.09 (m, 1H), $0.86(\mathrm{~s}, 9 \mathrm{H}),-0.01(\mathrm{~s}$, $6 \mathrm{H}) ;{ }^{13} \mathrm{C} \mathrm{NMR}\left(100 \mathrm{MHz}, \mathrm{CDCl}_{3}\right): \delta 172.1,170.1,158.5,130.3,128.8,113.3,63.7$, 56.6, 55.1, 52.4, 52.0, 46.0, 36.0, 27.6, 25.8, 18.2, -5.5; IR (neat, $\mathrm{cm}^{-1}$ ): 2952, 2856, 1730, 1613, 1514, 1435, 1248, 1179, 1128, 1036, 835, 810, 774, 668; HRMS-ESI: [M $\left.+\mathrm{NH}_{4}\right]^{+}$Calculated for $\mathrm{C}_{22} \mathrm{H}_{38} \mathrm{NO}_{6} \mathrm{Si}^{+}, 440.2463$; Found: 440.2464 . 


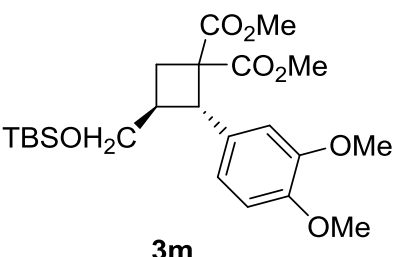

(2S,3S)-dimethyl 3-(((tert-butyldimethylsilyl)oxy)methyl)-2-(3,4dimethoxyphenyl)cyclobutane-1,1-dicarboxylate

Colorless liquid 3 h, 122.8 mg, (68\%, >99/1 dr); 99\% ee, determined by HPLC analysis: Daicel Chiralcel IA-3 column $(25 \mathrm{~cm})$, hexanes $/{ }^{i} \mathrm{PrOH}=98 / 2,0.6 \mathrm{~mL} / \mathrm{min}$, $280 \mathrm{~nm} ; \mathrm{t}_{\mathrm{r}}($ major $)=11.3 \mathrm{~min}, \mathrm{t}_{\mathrm{r}}($ minor $)=13.4 \mathrm{~min} ;[\alpha]_{\mathrm{D}}^{30.1}=-4.5^{\circ}(\mathrm{c}=1.12$, $\left.\mathrm{CHCl}_{3}\right) ;{ }^{1} \mathrm{H} \mathrm{NMR}\left(400 \mathrm{MHz}, \mathrm{CDCl}_{3}\right): \delta 6.85-6.75(\mathrm{~m}, 3 \mathrm{H}), 4.11(\mathrm{~d}, J=10.0 \mathrm{~Hz}, 1 \mathrm{H})$, $3.85(\mathrm{~s}, 3 \mathrm{H}), 3.83(\mathrm{~s}, 3 \mathrm{H}), 3.74(\mathrm{~s}, 3 \mathrm{H}), 3.63-3.55(\mathrm{~m}, 2 \mathrm{H}), 3.29(\mathrm{~s}, 3 \mathrm{H}), 3.04-2.96(\mathrm{~m}$, 1H), 2.68-2.63 (m, 1H), 2.13-2.08 (m, 1H), $0.85(\mathrm{~s}, 9 \mathrm{H}),-0.01(\mathrm{~s}, 6 \mathrm{H}) ;{ }^{13} \mathrm{C}$ NMR $(100$ $\left.\mathrm{MHz}, \mathrm{CDCl}_{3}\right): \delta 172.1,170.1,148.4,147.9,130.9,119.7,111.0,110.5,63.8,56.5$, 55.7, 52.4, 52.0, 46.4, 36.1, 27.6, 25.8, 18.2, -5.4(7), -5.4(8); IR (neat, $\mathrm{cm}^{-1}$ ): 2953, 2930, 2856, 1725, 1518, 1462, 1435, 1418, 1255, 1196, 1165, 1139, 1077, 1028, 834, 775, 671, 645; HRMS-ESI: $\left[\mathrm{M}+\mathrm{NH}_{4}\right]^{+}$Calculated for $\mathrm{C}_{23} \mathrm{H}_{40} \mathrm{NO}_{7} \mathrm{Si}^{+}, 470.2569$; Found: 470.2570 .

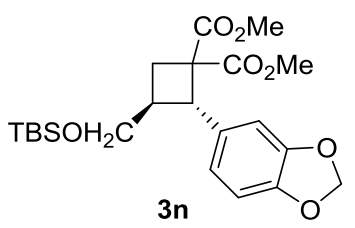

(2S,3S)-dimethyl 2-(benzo[d][1,3]dioxol-5-yl)-3-(((tertbutyldimethylsilyl)oxy)methyl)cyclobutane-1,1-dicarboxylate

Colorless liquid 2.5 h, 160.3 mg, (92\%, >99/1 dr); 96\% ee, determined by HPLC analysis: Daicel Chiralcel IC-3 column $(25 \mathrm{~cm})$, hexanes $/{ }^{i} \mathrm{PrOH}=95 / 5,0.7 \mathrm{~mL} / \mathrm{min}$, $300 \mathrm{~nm} ; \mathrm{t}_{\mathrm{r}}($ major $)=10.1 \mathrm{~min}, \mathrm{t}_{\mathrm{r}}($ minor $)=8.7 \mathrm{~min} ;[\alpha]_{\mathrm{D}}^{29.2}=-1.2^{\circ}\left(\mathrm{c}=1.32, \mathrm{CHCl}_{3}\right)$; ${ }^{1} \mathrm{H}$ NMR (400 MHz, $\left.\mathrm{CDCl}_{3}\right): \delta$ 6.83-6.82 (m, 1H), 6.76-6.70 (m, 2H), 5.91-5.90 (m, 2H), $4.09(\mathrm{~d}, J=10.4 \mathrm{~Hz}, 1 \mathrm{H}), 3.76(\mathrm{~s}, 3 \mathrm{H}), 3.62-3.54(\mathrm{~m}, 2 \mathrm{H}), 3.36(\mathrm{~s}, 3 \mathrm{H})$, 3.03-2.94 (m, 1H), 2.66-2.61 (m, 1H), 2.13-2.08 (m, 1H), $0.86(\mathrm{~s}, 9 \mathrm{H}),-0.01(\mathrm{~s}, 6 \mathrm{H})$; ${ }^{13} \mathrm{C} \mathrm{NMR}\left(100 \mathrm{MHz}, \mathrm{CDCl}_{3}\right): \delta 172.0,170.1,147.3,146.4,132.2,120.7,108.5,107.8$, 100.8, 63.6, 56.6, 52.4, 52.1, 46.5, 36.2, 27.6, 25.8, 18.2, -5.5; IR (neat, $\mathrm{cm}^{-1}$ ): 2953 , 2887, 2856, 1731, 1491, 1438, 1254, 1175, 1125, 1040, 937, 837, 776; HRMS-ESI: $\left[\mathrm{M}+\mathrm{NH}_{4}\right]^{+}$Calculated for $\mathrm{C}_{22} \mathrm{H}_{36} \mathrm{NO}_{7} \mathrm{Si}^{+}$, 454.2256; Found: 454.2259. 


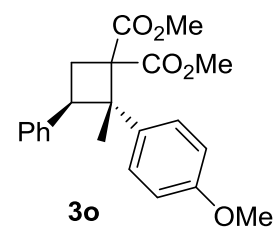

(2S,3R)-dimethyl 2-(4-methoxyphenyl)-2-methyl-3-phenylcyclobutane-1,1-dicarboxylate

Colorless liquid 12h, $108.4 \mathrm{mg}$, (74\%, >99/1 dr); 95\% ee, determined by HPLC analysis: Daicel Chiralcel OD-H column $(25 \mathrm{~cm})$, hexanes $/{ }^{i} \mathrm{PrOH}=98 / 2,0.6 \mathrm{~mL} / \mathrm{min}$, $240 \mathrm{~nm} ; \operatorname{tr}_{\mathrm{r}}($ major $)=15.3 \mathrm{~min}, \operatorname{tr}_{\mathrm{r}(}($ minor $)=17.2 \mathrm{~min} ;[\alpha]_{\mathrm{D}}^{28.3}=+63.4^{\circ}(\mathrm{c}=1.06$, $\left.\mathrm{CHCl}_{3}\right) ;{ }^{1} \mathrm{H} \mathrm{NMR}\left(400 \mathrm{MHz}, \mathrm{CDCl}_{3}\right): \delta 7.53(\mathrm{~d}, J=8.4 \mathrm{~Hz}, 2 \mathrm{H}), 7.35-7.22(\mathrm{~m}, 5 \mathrm{H})$, $6.88(\mathrm{~d}, J=8.8 \mathrm{~Hz}, 2 \mathrm{H}), 4.66(\mathrm{t}, J=9.6 \mathrm{~Hz}, 1 \mathrm{H}), 3.85(\mathrm{~s}, 3 \mathrm{H}), 3.81(\mathrm{~s}, 3 \mathrm{H}), 3.35(\mathrm{~s}$, 3H), 2.92-2.78 (m, 2H), $1.27(\mathrm{~s}, 3 \mathrm{H}) ;{ }^{13} \mathrm{C} \mathrm{NMR}\left(100 \mathrm{MHz}, \mathrm{CDCl}_{3}\right): \delta$ 170.9, 170.5, $158.0,139.7,137.8,128.2,127.3,126.5,113.3,60.1,55.2,52.2,40.7,29.1,23.3$; IR(neat, $\left.\mathrm{cm}^{-1}\right):$ 2952, 2837, 1729, 1610, 1513, 1433, 1245, 1180, 1143, 1076, 1030, 832, 763, 700; HRMS-ESI: $\left[\mathrm{M}+\mathrm{NH}_{4}\right]^{+}$Calculated for $\mathrm{C}_{22} \mathrm{H}_{28} \mathrm{NO}_{5}{ }^{+}, 386.1962$; Found: 386.1964 . 


\section{Total Synthesis of Piperarborenine B}

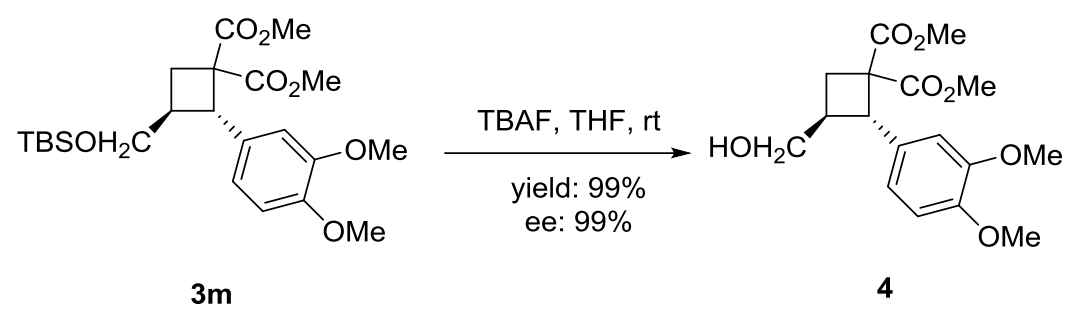

To a solution of $\mathbf{3 m}(3.17 \mathrm{~g}, 7 \mathrm{mmol})$ in THF $(30 \mathrm{~mL})$ at room temperature was added TBAF (1 M in THF, $10.5 \mathrm{~mL}, 1.5$ equiv. ), then the mixture was stirred at room temperature for $1 \mathrm{~h}$ until $\mathbf{3 m}$ was totally consumed. Saturated $\mathrm{NH}_{4} \mathrm{Cl}$ (aq.) (10 mL) was added to quench the reaction.The resulting solution was extracted with ethyl acetate $(3 \times 20 \mathrm{~mL})$. The organic phases were combined and dried over $\mathrm{Na}_{2} \mathrm{SO}_{4}$, filtered and concentrated. The residue was purified by flash chromatography (petroleum ether/ethyl acetate $=3 / 1 \sim 1 / 1$ ) on silica to afford the desired product 4 as a colorless liquid.

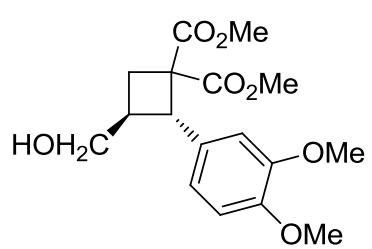

(2S,3S)-dimethyl 2-(3,4-dimethoxyphenyl)-3-(hydroxymethyl)cyclobutane-1,1-dicarboxylate

Colorless liquid 2.37g, (99\%); 99\% ee, determined by HPLC analysis: Daicel Chiralcel IA-3 column $(25 \mathrm{~cm})$, hexanes $/{ }^{i} \mathrm{PrOH}=90 / 10,0.7 \mathrm{~mL} / \mathrm{min}, 240 \mathrm{~nm} ; \mathrm{t}$ $($ major $)=26.2 \mathrm{~min}, \mathrm{t}($ minor $)=24.3 \mathrm{~min} ;[\alpha]_{\mathrm{D}}^{28.8}=+20.4^{\circ}\left(\mathrm{c}=0.73, \mathrm{CHCl}_{3}\right) ;{ }^{1} \mathrm{H}$ NMR (400 MHz, $\left.\mathrm{CDCl}_{3}\right): \delta$ 6.89-6.78 (m, 3H), $4.08(\mathrm{~d}, J=10.0 \mathrm{~Hz}, 1 \mathrm{H}), 3.87(\mathrm{~s}, 3 \mathrm{H})$, $3.85(\mathrm{~s}, 3 \mathrm{H}), 3.77(\mathrm{~s}, 3 \mathrm{H}), 3.71-3.67(\mathrm{~m}, 2 \mathrm{H}), 3.31(\mathrm{~s}, 3 \mathrm{H}), 3.11-3.08(\mathrm{~m}, 1 \mathrm{H})$, 2.79-2.74 (m, 1H), 2.10-2.05 (m, 1H), 1.55-1.53 (m, 1H); ${ }^{13} \mathrm{C}$ NMR (100 MHz, $\left.\mathrm{CDCl}_{3}\right): \delta 171.9,169.8,148.4,148.0,130.5,119.4,111.0,110.5,64.4,56.5,55.6,52.4$, 52.0, 46.9, 35.8, 28.0; IR (neat, $\mathrm{cm}^{-1}$ ): 3535, 2999, 2952, 2839, 1726, 1590, 1517, 1435, 1246, 1163, 1126, 1025, 860, 818, 762, 630; HRMS-ESI: $\left[\mathrm{M}+\mathrm{NH}_{4}\right]^{+}$ Calculated for $\mathrm{C}_{17} \mathrm{H}_{26} \mathrm{NO}_{7}^{+}$, 356.1704; Found: 356.1709 . 


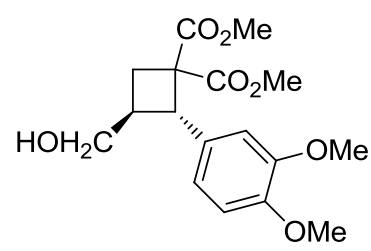

4

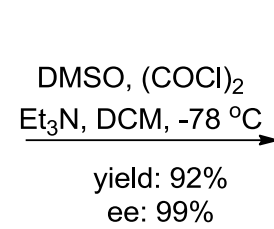

ee: $99 \%$

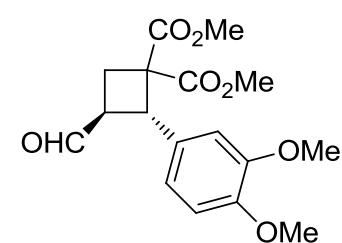

5

A DCM (5 mL) solution of DMSO (142 $\mu \mathrm{L}, 2.0 \mathrm{mmol}, 2$ equiv) was cooled to $-78{ }^{\circ} \mathrm{C}$, to which was added oxalyl chloride $(110 \mu \mathrm{L}, 1.3 \mathrm{mmol}, 1.3$ equiv).The reaction mixture was stirred at $-78{ }^{\circ} \mathrm{C}$ for 15 minutes. A solution of 4 (338.4 mg, 1.0 mmol) in DCM (3 mL) was added to the reaction mixture and stirred for another hour at $-78{ }^{\circ} \mathrm{C}$. Then $\mathrm{Et}_{3} \mathrm{~N}(0.4 \mathrm{~mL})$ was added and stirred for another 15 minutes at $-78{ }^{\circ} \mathrm{C}$. The reaction mixture was then stirred at $0{ }^{\circ} \mathrm{C}$ for 15 minutes before being quenched by water. The resulting solution was extracted with ethyl ether $(3 \times 10 \mathrm{~mL})$. The organic phases were combined and dried over $\mathrm{Na}_{2} \mathrm{SO}_{4}$, filtered and concentrated. The residue was purified by flash chromatography (petroleum ether/ethyl acetate $=$ 2/1) to afford the desired product 5 as a colorless liquid.

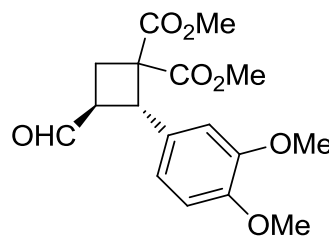

5

(2S,3S)-dimethyl 2-(3,4-dimethoxyphenyl)-3-formylcyclobutane-1,1-dicarboxylate

Colorless liquid $309.0 \mathrm{mg},(92 \%) ;[\alpha]_{\mathrm{D}}{ }^{26.5}=+29.8^{\circ}\left(\mathrm{c}=1.03, \mathrm{CHCl}_{3}\right) ;{ }^{1} \mathrm{H} \mathrm{NMR}$ (400 MHz, $\left.\mathrm{CDCl}_{3}\right): \delta 9.79(\mathrm{~d}, J=1.2 \mathrm{~Hz}, 1 \mathrm{H}), 6.90(\mathrm{~s}, 1 \mathrm{H}), 6.86-6.80(\mathrm{~m}, 2 \mathrm{H}), 4.40$ $(\mathrm{d}, J=10.4 \mathrm{~Hz}, 1 \mathrm{H}), 3.88(\mathrm{~s}, 3 \mathrm{H}), 3.86(\mathrm{~s}, 3 \mathrm{H}), 3.80-3.73(\mathrm{~m}, 4 \mathrm{H}), 3.35(\mathrm{~s}, 3 \mathrm{H})$, 2.88-2.83 (m, 1H), 2.50-2.45 (m, 1H); $\left.{ }^{13} \mathrm{C} \mathrm{NMR} \mathrm{(100} \mathrm{MHz,} \mathrm{CDCl}_{3}\right): \delta 200.3,171.1$, 169.3, 148.6, 148.4, 129.1, 119.2, 110.6(5), 110.5(6), 56.1, 55.7, 52.7, 52.4, 45.8, 44.1, 26.0; IR (neat, $\mathrm{cm}^{-1}$ ): 2954, 2838, 1726, 1590, 1517, 1436, 1242, 1198, 1164, 1145, 1123, 1025, 860, 817, 763, 633; HRMS-ESI: $\left[\mathrm{M}+\mathrm{NH}_{4}+\mathrm{MeOH}\right]^{+}$Calculated for $\mathrm{C}_{18} \mathrm{H}_{28} \mathrm{NO}_{8}{ }^{+}, 386.1809$; Found: 386.1812 . 


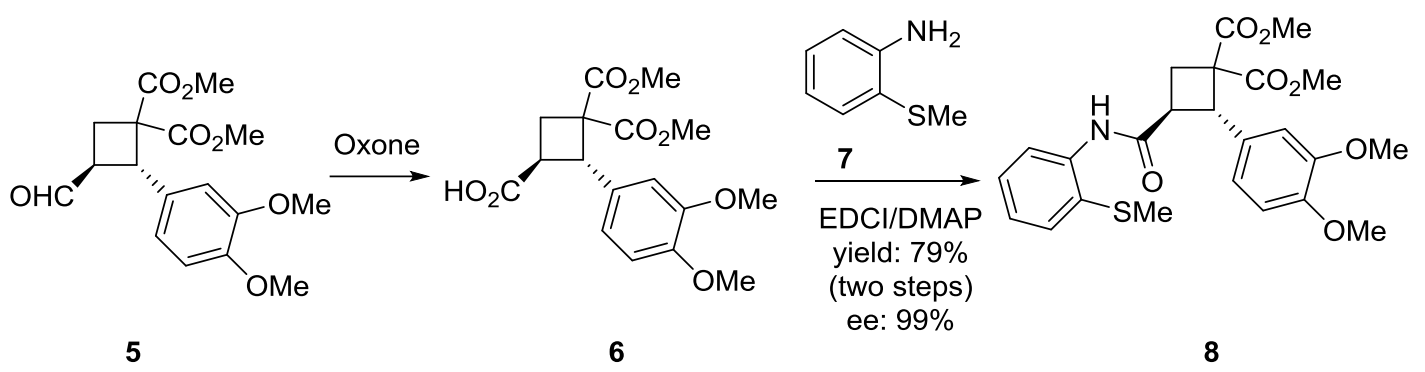

To a solution of 5 (1.20 g, $3.57 \mathrm{mmol})$ in DMF (20 mL) was added oxone (1.97 g, $3.21 \mathrm{mmol}, 0.9$ equiv.) and the mixture was stirred at room temperature for 1 hour. After the reaction was completed, hydrochloric acid $(1 \mathrm{~N})$ was added to dissolve the precipitate. The reaction mixture was extracted by ethyl ether, and the organic phase was combined, dried over $\mathrm{Na}_{2} \mathrm{SO}_{4}$, filtered and concentrated to give $\mathbf{6}$, which was used directly for next step reaction without any further purification.

To a dry Schlenk tube charged with 6 (3.57 mmol), 7 (0.547 g, $3.93 \mathrm{mmol}, 1.1$ equiv), DMAP (1.05 g, 8.59 mmol, 2.4 equiv), EDCI ( $0.821 \mathrm{~g}, 4.28 \mathrm{mmol}, 1.2$ equiv ) at room temperature under atmosphere of nitrogen was added DCM $(20 \mathrm{~mL})$, and the solution was stirred overnight. After the reaction was completed, chloride hydride $(1 \mathrm{~N}$, $10 \mathrm{~mL}$ ) was added. The reaction mixture was then extracted with DCM, and the organic phases were combined, dried over $\mathrm{Na}_{2} \mathrm{SO}_{4}$, filtered and concentrated to give a crude product.It was purified by flash chromatography on silica gel (hexane/ethyl acetate $=2 / 1)$ to give 8 .

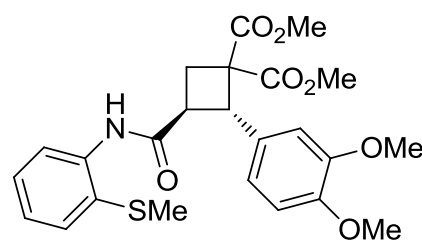

8

(2S,3S)-dimethyl 2-(3,4-dimethoxyphenyl)-3-((2(methylthio)phenyl)carbamoyl)cyclobutane-1,1-dicarboxylate

Light-yellow foam $1.34 \mathrm{~g},(79 \%) ;[\alpha]_{\mathrm{D}}{ }^{28.8}=+34.8^{\circ}\left(\mathrm{c}=1.21, \mathrm{CHCl}_{3}\right) ;{ }^{1} \mathrm{H}$ NMR (400 MHz, $\left.\mathrm{CDCl}_{3}\right): \delta 8.34-8.32(\mathrm{~m}, 2 \mathrm{H}), 7.43(\mathrm{~d}, J=7.2 \mathrm{~Hz}, 1 \mathrm{H}), 7.30-7.25(\mathrm{~m}, 1 \mathrm{H})$, 7.04-6.94 (m, 3H), $6.84(\mathrm{~d}, J=8.0 \mathrm{~Hz}, 1 \mathrm{H}), 4.48(\mathrm{~d}, J=10.8 \mathrm{~Hz}, 1 \mathrm{H}), 3.90(\mathrm{~s}, 3 \mathrm{H})$, $3.87(\mathrm{~s}, 3 \mathrm{H}), 3.80-3.76(\mathrm{~m}, 4 \mathrm{H}), 3.39(\mathrm{~s}, 3 \mathrm{H}), 2.94-2.89(\mathrm{~m}, 1 \mathrm{H}), 2.68-2.63(\mathrm{~m}, 1 \mathrm{H})$, 2.07 (s, 3H); ${ }^{13} \mathrm{C} \mathrm{NMR}\left(100 \mathrm{MHz}, \mathrm{CDCl}_{3}\right): \delta 170.1,169.8,169.7,148.9,147.7,138.1$, 133.3, 129.0, 126.4, 126.0, 124.6, 124.2, 120.1, 112.8, 110.4, 56.1, 56.0, 52.5, 52.4, 
45.0, 40.9, 28.3, 18.7; IR (neat, $\mathrm{cm}^{-1}$ ): 3252, 2940, 1731, 1717, 1658, 1605, 1577, 1535, 1515, 1461, 1438, 1386, 1350, 1317, 1256, 1196, 1161, 1057, 1039, 1019, 987, 964, 860, 821, 750, 684, 631; HRMS-ESI: $[\mathrm{M}+\mathrm{H}]^{+}$Calculated for $\mathrm{C}_{24} \mathrm{H}_{28} \mathrm{NO}_{7} \mathrm{~S}^{+}$, 474.1581; Found: 474.1583.

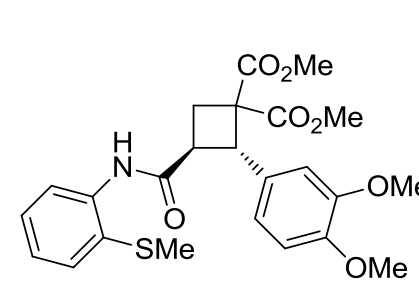

8

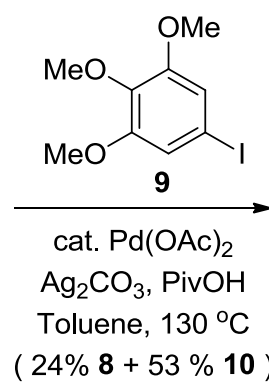

$(24 \% 8+53 \% 10)$

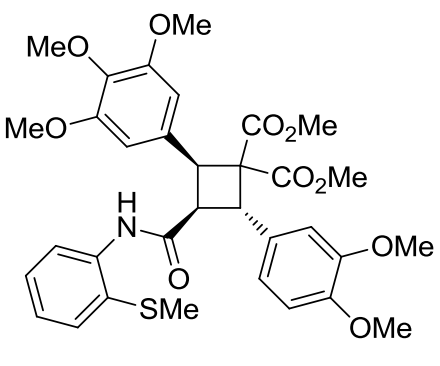

10

A Schlenk tube charged with 8 (1.06 g, $2.24 \mathrm{mmol}), 9$ (1.32 g, $4.48 \mathrm{mmol}, 2$ equiv), $\mathrm{Pd}(\mathrm{OAc})_{2}$ (151 mg, $0.672 \mathrm{mmol}, 0.3$ equiv), $\mathrm{Ag}_{2} \mathrm{CO}_{3}(927 \mathrm{mg}, 3.36 \mathrm{mmol}, 1.5$ equiv), PivOH (229 mg, $2.24 \mathrm{mmol}, 1$ equiv) and toluene (5 mL) was closed and heated at $130{ }^{\circ} \mathrm{C}$ with stirring for 72 hours. Then the reaction was cooled to room temperature and filtered through a pad of celite and eluted with DCM. The mixture was then concentrated to dryness that was purified by flash chromatography on silica gel (hexane/ ethyl acetate $=2 / 1$ ) to afford $\mathbf{1 0}$ as a light-yellow foam.

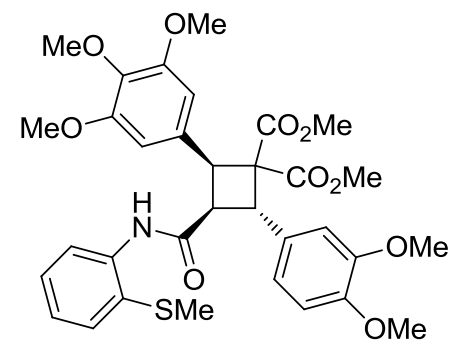

10

(2S,3R,4S)-dimethyl 2-(3,4-dimethoxyphenyl)-3-((2-(methylthio)phenyl)carbamoyl)-4-(3,4,5trimethoxyphenyl)cyclobutane-1,1-dicarboxylate

Light-yellow foam $758.0 \mathrm{mg}$, (yield: $53 \%$, b.r.s.m: $70 \%$ ); $[\alpha]_{\mathrm{D}}^{23.7}=-84.2^{\circ}(\mathrm{c}=$ 1.11, $\left.\mathrm{CHCl}_{3}\right) ;{ }^{1} \mathrm{H} \mathrm{NMR}\left(400 \mathrm{MHz}, \mathrm{CDCl}_{3}\right): \delta 8.19-8.16(\mathrm{~m}, 2 \mathrm{H}), 7.37(\mathrm{~d}, J=7.6 \mathrm{~Hz}$, 1H), 7.21-7.17 (m, 1H), 7.00-6.83 (m, 4H), $6.57(\mathrm{~s}, 2 \mathrm{H}), 5.22(\mathrm{~d}, J=11.2 \mathrm{~Hz}, 1 \mathrm{H})$, 4.62-4.59 (m, 1H), 4.28-4.22 (m, 1H), $3.92(\mathrm{~s}, 3 \mathrm{H}), 3.87$ (s, 3H), 3.78 (s, 6H), 3.71 (s, $3 \mathrm{H}), 3.35$ (s, 3H), 3.33 (s, 3H), 2.03 (s, 3H); $\left.{ }^{13} \mathrm{C} \mathrm{NMR} \mathrm{(100} \mathrm{MHz,} \mathrm{CDCl}_{3}\right): \delta$ 169.5, $169.1,168.6,152.9,148.7,148.5,138.1,137.3,133.4,131.4,129.3,129.1,124.4$, $124.1,119.8,119.2,111.1,110.7,105.8,62.3,60.7,56.1,55.8,52.6,52.2,47.3,45.0$, 
43.9, 18.9; IR (neat, $\mathrm{cm}^{-1}$ ): 2950, 1727, 1687, 1588, 1512, 1461, 1432, 1247, 1124 , 1026, 759; HRMS-ESI: $[\mathrm{M}+\mathrm{H}]^{+}$Calculated for $\mathrm{C}_{33} \mathrm{H}_{38} \mathrm{NO}_{10} \mathrm{~S}^{+}, 640.2211$; Found: 640.2209 .

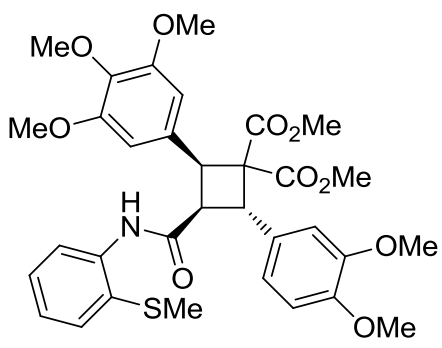

10

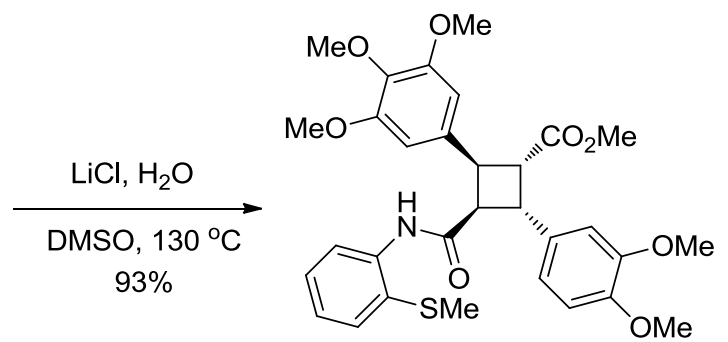

11

To a dry Schlenk tube was added $10(524.0 \mathrm{mg}, 0.8 \mathrm{mmol}), \mathrm{LiCl}(339 \mathrm{mg}, 8$ mmol, 10 equiv.), $\mathrm{H}_{2} \mathrm{O}$ (144 mg, $8 \mathrm{mmol}, 10$ equiv.) and DMSO (10 mL). The reaction was stirred at $130{ }^{\circ} \mathrm{C}$ for 48 hours. After removal of the solvent, the residue was purified by flash chromatography on silica gel (hexane/ethyl acetate $=2 / 1 \sim 1 / 1$ ) to yield $\mathbf{1 1}$ as a light-yellow foam.

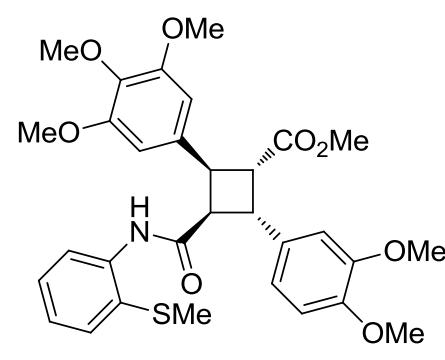

11

(1R,2R,3R,4R)-methyl 2-(3,4-dimethoxyphenyl)-3-((2-(methylthio)phenyl)carbamoyl)-4-(3,4,5trimethoxyphenyl)cyclobutanecarboxylate

Light-yellow foam $435.0 \mathrm{mg},(93 \%) ;[\alpha]_{\mathrm{D}}{ }^{27.7}=+21.6^{\circ}\left(\mathrm{c}=1.11, \mathrm{CHCl}_{3}\right) ;{ }^{1} \mathrm{H}$ NMR (400 MHz, $\left.\mathrm{CDCl}_{3}\right): \delta 8.17(\mathrm{~d}, J=8.4 \mathrm{~Hz}, 1 \mathrm{H}), 8.00(\mathrm{~s}, 1 \mathrm{H}), 7.36(\mathrm{~d}, J=7.6 \mathrm{~Hz}$, 1H), 7.22-7.18 (m, 1H), 7.00-6.95 (m, 2H), 6.89-6.85 (m, 2H), 6.59 (s, 2H), 4.60-4.56 (m, 1H), 4.49-4.45 (m, 1H), 3.97-3.86 (m, 8H), 3.78 (s, 6H), 3.67 (s, 3H), 3.39 (s, 3H), $2.10(\mathrm{~s}, 3 \mathrm{H}) ;{ }^{13} \mathrm{C} \mathrm{NMR}\left(100 \mathrm{MHz}, \mathrm{CDCl}_{3}\right): \delta 172.4,169.3,153.1,148.6,148.0,138.0$, 137.1, 134.1, 133.1, 131.4, 128.8, 124.5, 124.2, 119.7, 119.3, 111.2, 110.8, 104.7, 60.6, 56.0, 55.9, 55.8, 51.6, 49.7, 47.6, 42.6, 41.0, 18.8; IR (neat, $\mathrm{cm}^{-1}$ ): 3315, 2924, 2851, 1723, 1676, 1579, 1511, 1453, 1429, 1378, 1340, 1321, 1303, 1257, 1231, 1193, 1160 , 1124, 1025, 935, 848, 808, 751, 683, 648, 635; HRMS-ESI: $[\mathrm{M}+\mathrm{H}]^{+}$Calculated for $\mathrm{C}_{31} \mathrm{H}_{36} \mathrm{NO}_{8} \mathrm{~S}^{+}, 582.2156$; Found: 582.2158. 


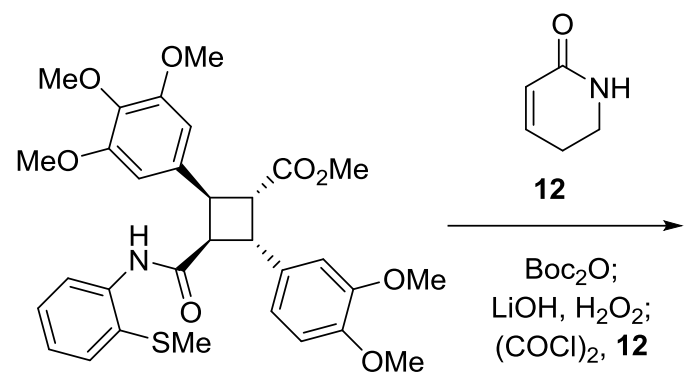

11

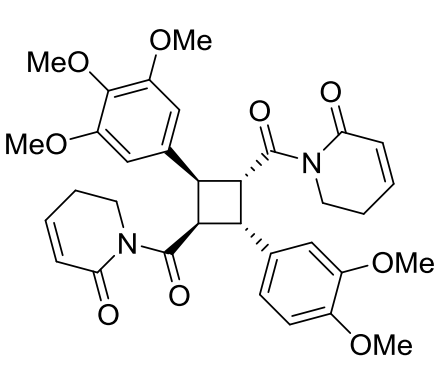

piperarborenine $B$

To a solution of $11(150.8 \mathrm{mg}, 0.26 \mathrm{mmol})$ in $\mathrm{MeCN}(2.5 \mathrm{~mL})$ was added (Boc) $)_{2} \mathrm{O}$ (84.8 mg, $0.39 \mathrm{mmol}, 1.5$ equiv.) and DMAP (3.2 mg, $0.026 \mathrm{mmol}, 0.1$ equiv.). The reaction was stirred at room temperature for 3 hours. After removel of the solvent, the residue was purified by flash chromatography on silica gel (hexane/ethyl acetate $=3 / 1)$ to give the protected 11 . Then $56.3 \mathrm{mg}(0.083 \mathrm{mmol})$ of the protected 11 was dissolved in THF (4 mL) and $\mathrm{H}_{2} \mathrm{O}(2 \mathrm{~mL})$, to which was added $\mathrm{LiOH}(11.9 \mathrm{mg}$, 0.50 mmol, 6 equiv. $)$ and $30 \% \mathrm{H}_{2} \mathrm{O}_{2}(0.83 \mathrm{mmol}, 10$ equiv. $)$ at $0{ }^{\circ} \mathrm{C}$. The reaction mixture was warmed to room temperature and stirred for 1 hour, and then heated to 50 ${ }^{\circ} \mathrm{C}$ and stirred for another 24 hours. The reaction was then cooled to room temperature and diluted with ethyl acetate.A saturated $\mathrm{Na}_{2} \mathrm{SO}_{3}$ solution was added and the aqueous phase was washed with ethyl acetate before acidified using hydrochloric acid (3 N, 10 $\mathrm{mL})$. The resulting solution was extracted with a mixed solvent of $\mathrm{CHCl}_{3} /{ }^{i} \mathrm{PrOH}(7 / 3$ in $\mathrm{V} / \mathrm{V}, 5 \times 10 \mathrm{~mL}$ ). The organic portions were combined and dried over $\mathrm{Na}_{2} \mathrm{SO}_{4}$, filtered and concentrated to give a white solid diacid. To a solution of the prepared diacid $(22.3 \mathrm{mg}, 0.05 \mathrm{mmol})$ in THF $(2 \mathrm{~mL})$ was added dropwise DMF $(1 \mu \mathrm{L})$ and $(\mathrm{COCl})_{2}$, and the reaction was stirred at room temperature for 2 hours. After removal of THF and addition of $4 \AA$ MS (100 mg), 12 (14.6 mg, $0.15 \mathrm{mmol}, 3$ equiv) and toluene $(2 \mathrm{~mL})$, the reaction was stirred at $80^{\circ} \mathrm{C}$ overnight. The reaction mixture was then concentrated and purified by flash chromatography on silica gel (hexane/ethyl acetate $=1 / 1 \sim 1 / 2$ ) to give piperarborenine $\mathrm{B}$ as a white solid. 


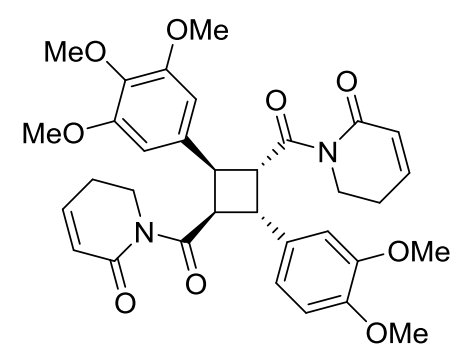

piperarborenine $B$

White solid $26.8 \mathrm{mg}$, (69\%); 99\% ee, determined by HPLC analysis: Daicel Chiralcel IA-3 column $(25 \mathrm{~cm})$, hexanes $/{ }^{i} \mathrm{PrOH}=70 / 30,0.8 \mathrm{~mL} / \mathrm{min}, 254 \mathrm{~nm} ; \mathrm{t}$ $($ major $)=47.6 \mathrm{~min}, \mathrm{t}($ minor $)=42.4 \mathrm{~min} ;[\alpha]_{\mathrm{D}}{ }^{28.6}=+5.8^{\circ}\left(\mathrm{c}=1.00, \mathrm{CH}_{2} \mathrm{Cl}_{2}\right) ;{ }^{1} \mathrm{H}$ $\operatorname{NMR}\left(400 \mathrm{MHz}, \mathrm{CDCl}_{3}\right): \delta 6.89\left(\mathrm{dd}, J_{1}=8.4 \mathrm{~Hz}, J_{2}=2.0 \mathrm{~Hz}, 1 \mathrm{H}\right), 6.82-6.76(\mathrm{~m}, 2 \mathrm{H})$, 6.68-6.64 (m, 2H), $6.52(\mathrm{~s}, 2 \mathrm{H}), 5.78-5.74(\mathrm{~m}, 2 \mathrm{H}), 4.92(\mathrm{dd}, J=11.6,7.2 \mathrm{~Hz}, 1 \mathrm{H})$, $4.89(\mathrm{dd}, J=11.6,7.2 \mathrm{~Hz}, 1 \mathrm{H}), 4.76(\mathrm{dd}, J=11.2,8.0 \mathrm{~Hz}, 1 \mathrm{H}), 4.74(\mathrm{dd}, J=11.2,7.2$ Hz), 3.90-3.89 (m, 1H) , $3.86(\mathrm{~s}, 3 \mathrm{H}), 3.85(\mathrm{~s}, 6 \mathrm{H}), 3.84(\mathrm{~s}, 3 \mathrm{H}), 3.79-3.74(\mathrm{~m}, 4 \mathrm{H})$, 3.48-3.33 (m, 2H), 2.09-2.02 (m, 2H), 1.69-1.60 (m, 2H); ${ }^{13} \mathrm{C}$ NMR (100 MHz, $\left.\mathrm{CDCl}_{3}\right): \delta 174.1(1), 174.0(9), 164.4(2), 164.4(1), 152.7,148.1,147.7,145.0(8)$, 145.0(6), 136.4(2), 136.3(8), 133.1, 125.4(3), 125.3(5), 119.8, 111.9, 110.8, 105.0, 60.7, 56.1, 56.0, 55.8, 51.3(9), 51.3(7), 42.2, 41.6, 40.8, 40.8, 24.1; IR (neat, $\mathrm{cm}^{-1}$ ): 3610, 2939, 2837, 1684, 1588, 1512, 1463, 1422, 1385, 1307, 1245, 1219, 1184, 1125, 1025, 910, 852, 814, 765, 731, 701, 636; HRMS-ESI: $[\mathrm{M}+\mathrm{H}]^{+}$Calculated for $\mathrm{C}_{33} \mathrm{H}_{37} \mathrm{~N}_{2} \mathrm{O}_{9}{ }^{+}$, 605.2494; Found: 605.2491. 
Table S4: ${ }^{1} \mathrm{H}$ NMR in $\mathrm{CDCl}_{3}$ at $400 \mathrm{M} \mathrm{Hz}$

\begin{tabular}{|c|c|c|c|c|c|}
\hline & $\begin{array}{l}5 \text { aromatic } \\
\text { protons }\end{array}$ & $\begin{array}{l}4 \text { olefin } \\
\text { protons }\end{array}$ & $\begin{array}{l}4 \text { cyclobutane } \\
\text { protons }\end{array}$ & $\begin{array}{l}5 \quad \mathrm{MeO} \\
\text { groups }\end{array}$ & $\begin{array}{l}8 \\
\text { six-membered } \\
\text { ring protons }\end{array}$ \\
\hline $\begin{array}{l}\text { Synthesizec } \\
\delta=\end{array}$ & $\begin{array}{l}6.89(\mathrm{dd}, J \\
=8.4, \quad 2.0 \\
\mathrm{~Hz}, \quad 1 \mathrm{H}), \\
6.82-6.76 \\
(\mathrm{~m}, \quad 2 \mathrm{H}), \\
6.52 \quad(\mathrm{~s}, \\
2 \mathrm{H}),\end{array}$ & $\begin{array}{c}6.68-6.64 \\
(\mathrm{~m}, 2 \mathrm{H}), \\
5.78-5.74 \\
(\mathrm{~m}, 2 \mathrm{H})\end{array}$ & $\begin{array}{c}4.92(\mathrm{dd}, J= \\
11.6,7.2 \mathrm{~Hz}, \\
1 \mathrm{H}), 4.89(\mathrm{dd}, \\
J=11.6,7.2 \\
\mathrm{~Hz}, 1 \mathrm{H}), 4.76 \\
(\mathrm{dd}, J=11.2, \\
8.0 \mathrm{~Hz}, 1 \mathrm{H}), \\
4.74(\mathrm{dd}, J= \\
11.2,7.2 \mathrm{~Hz})\end{array}$ & $\begin{array}{l}3.86 \quad(\mathrm{~s}, \\
3 \mathrm{H}), \quad 3.85 \\
(\mathrm{~s}, \quad 6 \mathrm{H}), \\
3.84 \quad(\mathrm{~s}, \\
3 \mathrm{H}), \\
3.79-3.74 \\
(\mathrm{~m}, 3 \mathrm{H}),\end{array}$ & $\begin{array}{lr}3.90-3.89 & (\mathrm{~m}, \\
1 \mathrm{H}), 3.79-3.74 \\
(\mathrm{~m}, & 1 \mathrm{H}), \\
3.48-3.33 & (\mathrm{~m}, \\
2 \mathrm{H}), & 2.09-2.02 \\
(\mathrm{~m}, & 2 \mathrm{H}), \\
1.69-1.60 & (\mathrm{~m}, \\
2 \mathrm{H}) ; & \end{array}$ \\
\hline $\begin{array}{l}\text { Isolated } \\
\delta= \\
\text { (Helv. } \\
\text { Chim. Acta } \\
\text { 2004, } 87, \\
463-468 .)\end{array}$ & $\begin{array}{l}6.88(\mathrm{dd}, J \\
=8.4,2.0 \\
\mathrm{~Hz}, 1 \mathrm{H}), \\
6.82(\mathrm{~d}, J \\
=2.0 \mathrm{~Hz}, \\
1 \mathrm{H}), 6.77 \\
(\mathrm{~d}, J=8.4 \\
\mathrm{Hz}, 1 \mathrm{H}), \\
6.52(\mathrm{~s}, \\
2 \mathrm{H})\end{array}$ & $\begin{array}{l}6.65(\mathrm{~m}, \\
2 \mathrm{H}), 5.76 \\
(\mathrm{~m}, 2 \mathrm{H})\end{array}$ & $\begin{array}{l}4.89(\mathrm{dd}, J= \\
11.4,7.4 \mathrm{~Hz}, \\
1 \mathrm{H}), 4.92(\mathrm{dd}, \\
J=11.4,7.4 \\
\mathrm{~Hz}, 1 \mathrm{H}), 4.73 \\
(\mathrm{dd}, J=11.4, \\
7.4 \mathrm{~Hz}, 1 \mathrm{H}), \\
4.76(\mathrm{dd}, J= \\
11.4,7.4 \mathrm{~Hz})\end{array}$ & $\begin{array}{lr}3.86 & (\mathrm{~s}, \\
3 \mathrm{H}), & 3.85 \\
(\mathrm{~s}, & 6 \mathrm{H}), \\
3.84 & (\mathrm{~s}, \\
3 \mathrm{H}), & 3.79 \\
(\mathrm{~s}, 3 \mathrm{H}),\end{array}$ & $\begin{array}{l}3.82(\mathrm{~m}, 2 \mathrm{H}), \\
3.41(\mathrm{~m}, 2 \mathrm{H}), \\
2.06(\mathrm{~m}, 2 \mathrm{H}), \\
1.65(\mathrm{~m}, 2 \mathrm{H}),\end{array}$ \\
\hline
\end{tabular}

Table S5: ${ }^{13} \mathrm{C}$ NMR in $\mathrm{CDCl}_{3}$ at $100 \mathrm{M} \mathrm{Hz}$

\begin{tabular}{|c|c|c|c|}
\hline & $\begin{array}{l}4 \quad \text { carbonyl } \\
\text { carbons }\end{array}$ & $\begin{array}{l}10 \text { aryl carbons signals and } \\
4 \text { olefin carbons }\end{array}$ & $\begin{array}{l}11 \text { aliphatic } \\
\text { carbons signals }\end{array}$ \\
\hline $\begin{array}{l}\text { Synthesized } \\
\delta=\end{array}$ & $\begin{array}{l}174.1(1), \\
174.0(9), \\
164.4(2), \\
164.4(1),\end{array}$ & $\begin{array}{lrr}152.7, & 148.1, & 147.7, \\
145.0(8), & 145.0(6), & 136.4(2), \\
136.3(8), & 133.1, & 125.4(3), \\
125.3(5), & 119.8, & 111.9, \\
110.8,105.0,\end{array}$ & $\begin{array}{l}60.7,56.1, \quad 56.0, \\
55.8, \quad 51.3(9), \\
51.3(7), 42.2,41.6, \\
40.8,40.8,24.1 ;\end{array}$ \\
\hline $\begin{array}{l}\text { Isolated } \\
\delta= \\
\text { (Helv. Chim. Acta } \\
\text { 2004, } \\
\text { 463-468.) }\end{array}$ & $\begin{array}{l}174.1 \\
164.4\end{array}$ & $\begin{array}{l}\text { 152.7, 148.3, } 147.8 \text { 145.0, } \\
\text { 136.6, 136.4, 133.1, 125.4, } \\
\text { 125.5, 120.0, 112.0, 111.0, } \\
105.2\end{array}$ & $\begin{array}{l}60.7,56.1,56.1 \\
56.0,55.8,51.4 \\
42.2,41.6,40.8 \\
40.7,24.1\end{array}$ \\
\hline
\end{tabular}




\section{X-ray Data of 3d}

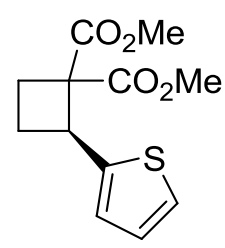

3d

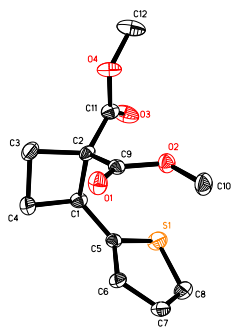

\begin{tabular}{|c|c|c|}
\hline Empirical formula & \multicolumn{2}{|l|}{$\mathrm{C} 12 \mathrm{H} 14 \mathrm{O} 4 \mathrm{~S}$} \\
\hline Formula weight & \multicolumn{2}{|l|}{254.29} \\
\hline Temperature, Wavelength & \multicolumn{2}{|c|}{$296.15 \mathrm{~K}, 0.71073 \AA$} \\
\hline Crystal system, Space group & \multicolumn{2}{|l|}{ Monoclinic, $\mathrm{P} 2{ }_{1}$} \\
\hline \multirow[t]{3}{*}{ Unit cell dimensions } & $\mathrm{a}=8.198(4) \AA$ & $\alpha=90^{\circ}$. \\
\hline & $\mathrm{b}=9.110(5) \AA$ & $\beta=92.976(10)^{\circ}$ \\
\hline & $\mathrm{c}=8.427(4) \AA$ & $\gamma=90^{\circ}$. \\
\hline Volume, Z & \multicolumn{2}{|l|}{$628.5(6) \AA^{3}, 2$} \\
\hline Density (calculated) & \multicolumn{2}{|l|}{$1.344 \mathrm{Mg} / \mathrm{m}^{3}$} \\
\hline Absorption coefficient, $\mathrm{F}(000)$ & \multicolumn{2}{|l|}{$0.257 \mathrm{~mm}^{-1}, 268$} \\
\hline Crystal size & \multicolumn{2}{|c|}{$0.38 \times 0.29 \times 0.25 \mathrm{~mm}^{3}$} \\
\hline Theta range for data collection & \multicolumn{2}{|l|}{2.420 to $25.995^{\circ}$. } \\
\hline Index ranges & \multicolumn{2}{|c|}{$-8<=\mathrm{h}<=10,-11<=\mathrm{k}<=11,-10<=1<=10$} \\
\hline Reflections collected & \multicolumn{2}{|l|}{3781} \\
\hline Independent reflections & \multicolumn{2}{|c|}{$2190[\mathrm{R}(\mathrm{int})=0.0443]$} \\
\hline Completeness to theta $=26.000^{\circ}$ & \multicolumn{2}{|l|}{$97.9 \%$} \\
\hline Absorption correction & \multicolumn{2}{|c|}{ Semi-empirical from equivalents } \\
\hline Max. and min. transmission & \multicolumn{2}{|c|}{0.7461 and 0.2867} \\
\hline Refinement method & \multicolumn{2}{|c|}{ Full-matrix least-squares on $\mathrm{F}^{2}$} \\
\hline Data / restraints / parameters & \multicolumn{2}{|l|}{$2190 / 1 / 156$} \\
\hline Goodness-of-fit on $\mathrm{F}^{2}$ & \multicolumn{2}{|l|}{1.059} \\
\hline Final $R$ indices $[I>2 \operatorname{sigma}(I)]$ & \multicolumn{2}{|c|}{$\mathrm{R} 1=0.0776, \mathrm{wR} 2=0.2140$} \\
\hline $\mathrm{R}$ indices (all data) & \multicolumn{2}{|c|}{$\mathrm{R} 1=0.0836, \mathrm{wR} 2=0.2187$} \\
\hline Absolute structure parameter & \multicolumn{2}{|l|}{$0.01(11)$} \\
\hline Extinction coefficient & \multicolumn{2}{|l|}{$\mathrm{n} / \mathrm{a}$} \\
\hline Largest diff. peak and hole & \multicolumn{2}{|c|}{0.488 and -0.678 e. $\AA^{-3}$} \\
\hline
\end{tabular}




\section{VCD and IR Experiment}

VCD and IR spectra were measured using a BioTools ChiralIR-2X FT-VCD spectrometer, equipped with a photoelastic modulation and a mercury cadmium tellurium detector. $7.3 \mathrm{mg}$ of $\mathbf{3 a}$ (or $4.9 \mathrm{mg}$ of $\mathbf{3 g}$ ) were dissolved in $150 \mu \mathrm{L}$ of $\mathrm{CDCl}_{3}$ and placed in $\mathrm{a} \mathrm{BaF}_{2}$ cell with a pathlength of $75 \mu \mathrm{m}$. Data were acquired at a resolution of $4 \mathrm{~cm}^{-1}$ for $3 \mathrm{~h}$ (or $6 \mathrm{~h}$ for $\mathbf{3 g}$ ). The corresponding racemates were measured under the same conditions to obtain VCD baseline.

\section{VCD and IR calculations}

The assignment of absolute configuration with VCD method is based on comparisons of the experimental spectra with the theoretical curve obtained by density functional theory (DFT) calculations. Initially, molecular models of $(R)-\mathbf{3 a}$ and $(R, R)-\mathbf{3 g}$ were built. Monte Carlo conformational searching at the molecular mechanic force field MMFF94 level were then performed using Compute VOA (BioTools Inc., Jupiter, FL). Within a $10 \mathrm{kcal} / \mathrm{mol}$ energy window, twenty-one energetically distinct conformers for $(R)-\mathbf{3 a}$ and twenty for $(R, R)-\mathbf{3 g}$ were predicted.

Geometry optimization of all conformers were carried out using the B3LYP hybrid density functional and 6-31G(d) basis set with Gaussian 09 (Gaussian Inc., Wallingford, CT). Five different conformers of $(R)-\mathbf{3 a}$, contributing to $99.6 \%$ of the total conformational population, were found in a $2.6 \mathrm{kcal} / \mathrm{mol}$ range. VCD and IR frequencies of the conformers were then calculated at the same level of theory. For $(R, R)-3 \mathrm{~g}$, three conformers in a $1.6 \mathrm{kcal} / \mathrm{mol}$ energy gap accounting for $98.3 \%$ of the total population were found and further calculated. The most stable conformers of $(R)-3 \mathbf{a}$ and $(R, R)-3 \mathbf{g}$ together with their Boltzmann population based on B3LYP/6-31G(d) relative energies are shown in Fig S2 and Fig S3. Boltzmann-population-weighted composite VCD and IR spectra were generated by Compute VOA. Theoretical curves of $(S)-\mathbf{3 a}$ and $(S, S)-\mathbf{3 g}$ were then deduced from $(R)-3 \mathbf{a}$ and $(R, R)-3 \mathbf{g}$.

Fig S4 to Fig S7 show the observed and calculated VCD and IR spectra of 3a 
and $3 \mathrm{~g}$ over the range of $950-1850 \mathrm{~cm}^{-1}$. A scale factor of 0.97 has been applied to the calculated frequencies to overcome a systematic overestimation of the molecular force constant values by DFT. According to the good agreement between the corresponding spectra, 3a is assigned.to have $(R)$ configuration unambiguously. The experimental VCD spectrum of $\mathbf{3 g}$ is in much better agreement with the calculated VCD spectrum of $(\mathrm{S}, S)-\mathbf{3 g}$ than $(R, R)-\mathbf{3 g}$. As a result, the absolute configuration of $\mathbf{3 g}$ is assigned as $(\mathrm{S}, S)$.

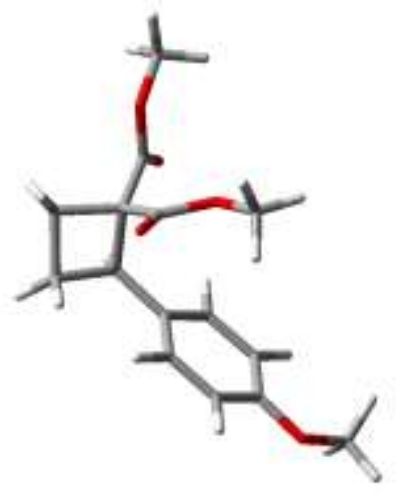

3a-1 $(78.7 \%)$

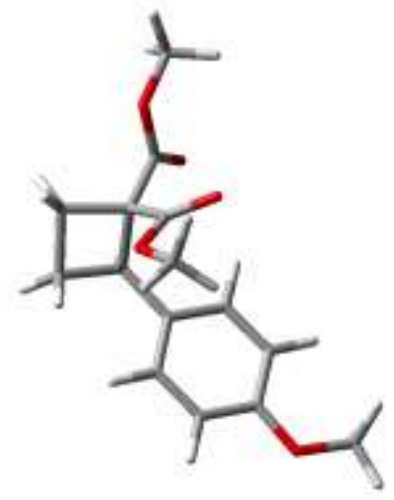

3a-2 $(8.4 \%)$

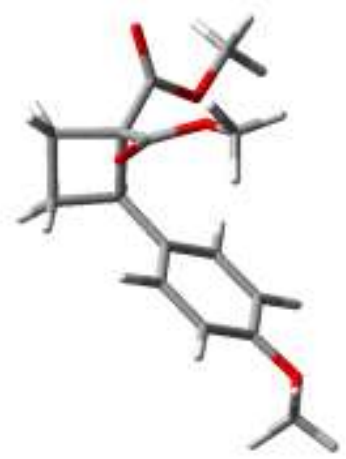

$\mathbf{3 a - 3}(6.1 \%)$

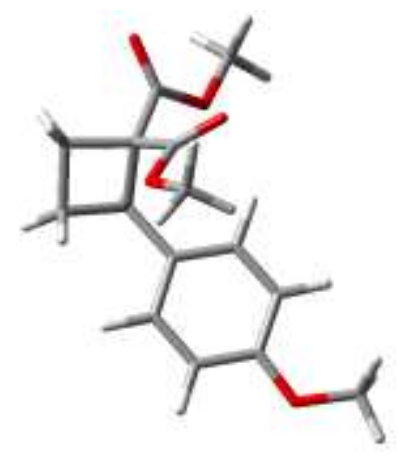

3a-5 $(1.1 \%)$

Figure S2 DFT// B3LYP /6-31G(d) minimum energy structures of $(R)-3 \mathbf{a}$ 


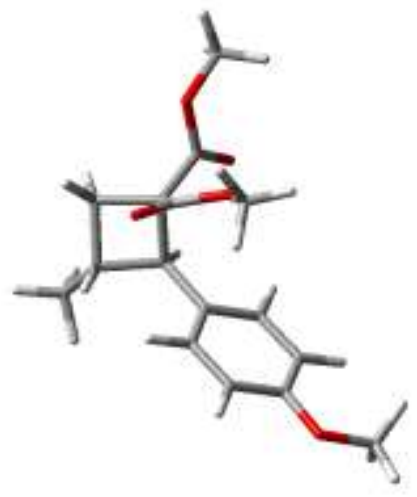

3g-1 $(85.3 \%)$

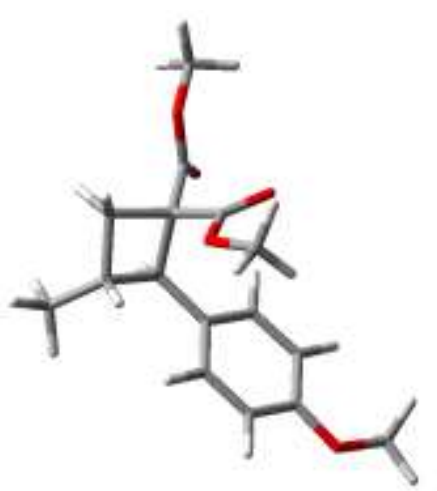

3g-2 (7.1\%)

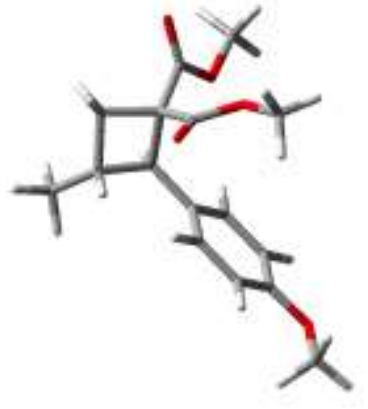

3g-3 $(5.9 \%)$

Figure S3 DFT// B3LYP /6-31G(d) minimum energy structures of $(R, R)-3 \mathbf{g}$

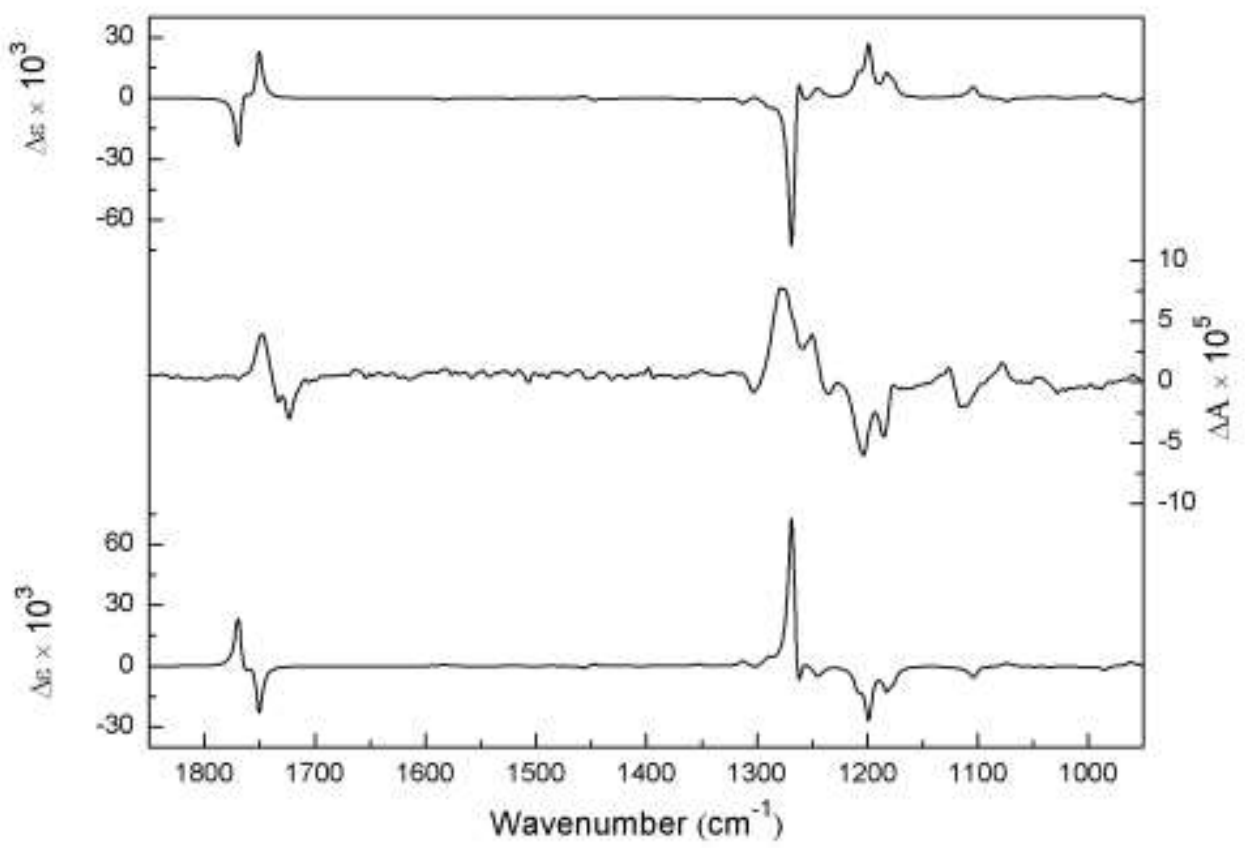

Figure S4 Comparison of the experimental VCD spectrum of 3a (center) and the calculated VCD spectra of (S)-3a (top) and (R)-3a (bottom) 


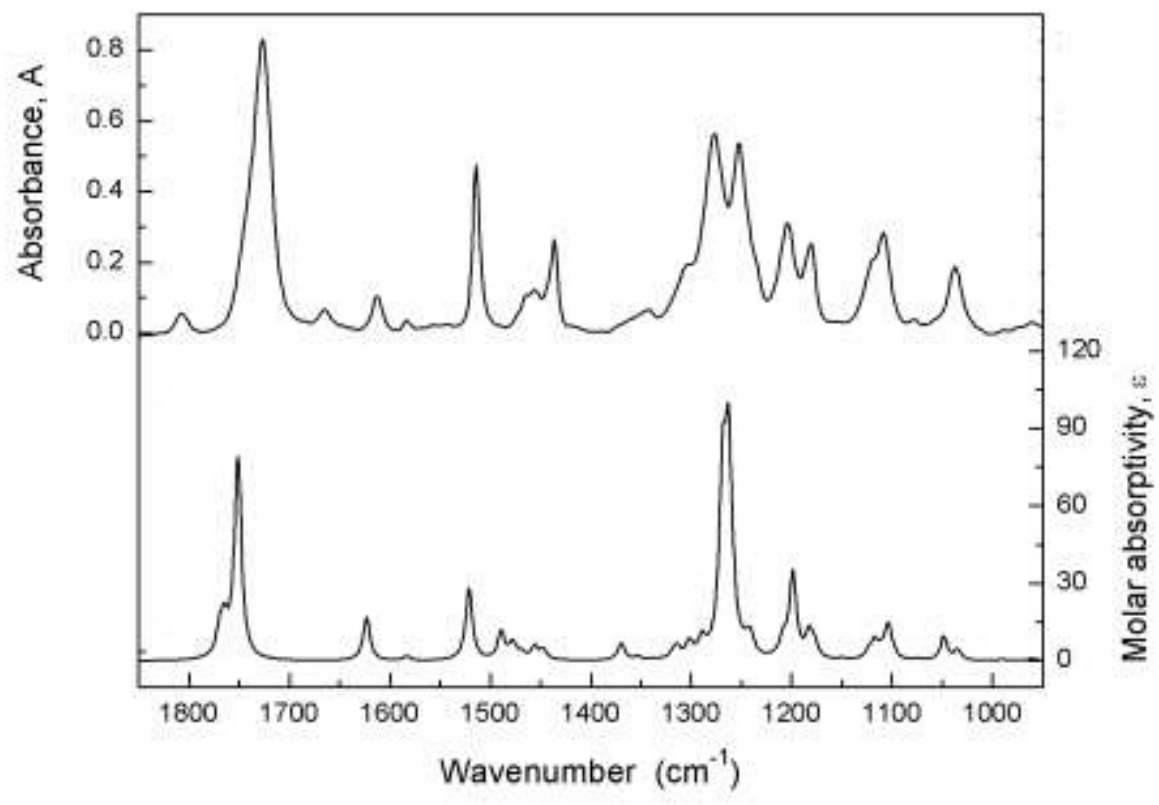

Figure S5 Comparison of the experimental IR spectrum of 3a (top) and the calculated IR spectra for (S)-3a and (R)-3a (bottom)

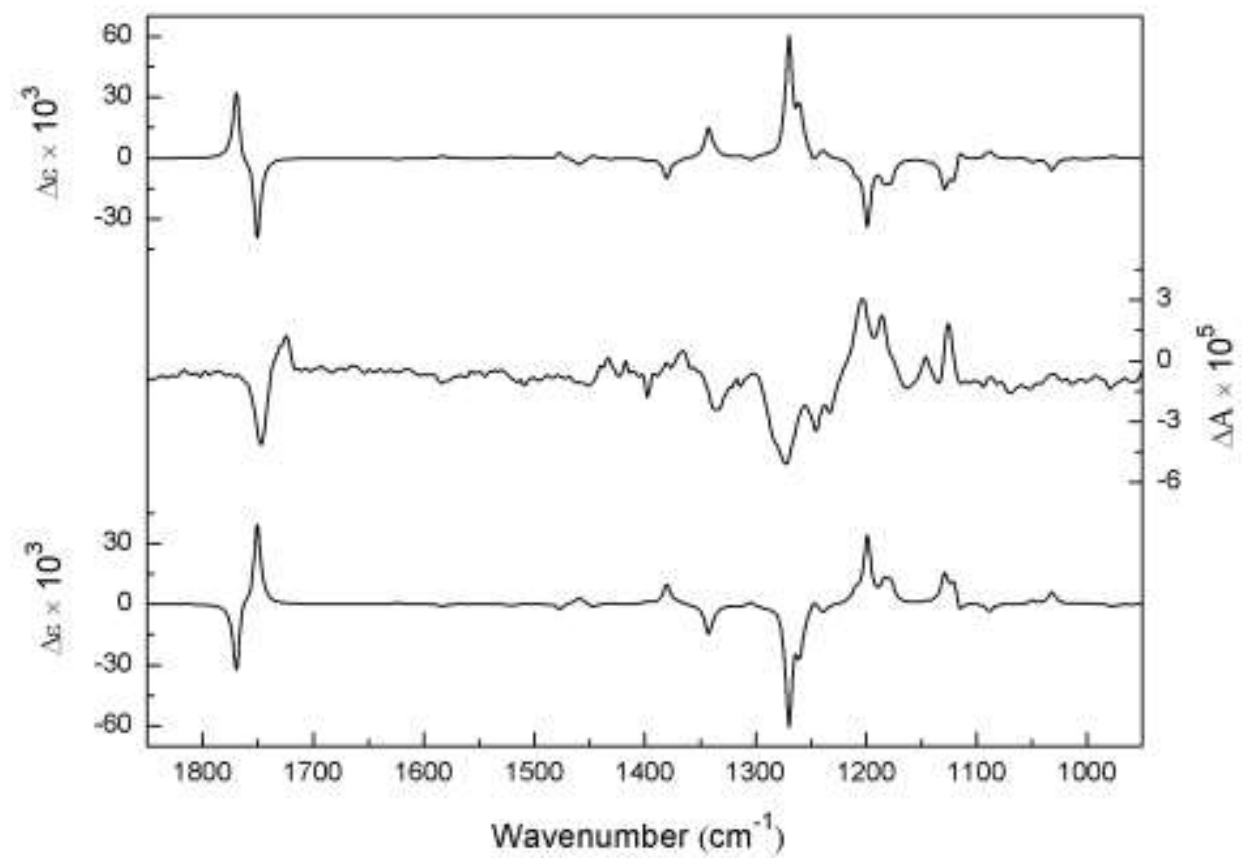

Figure S6 Comparison of the experimental VCD spectrum of $\mathbf{3 g}$ (center) and the calculated VCD spectra of $(R, R)-\mathbf{3 g}$ (top) and $(S, S)$ - 3g (bottom) 


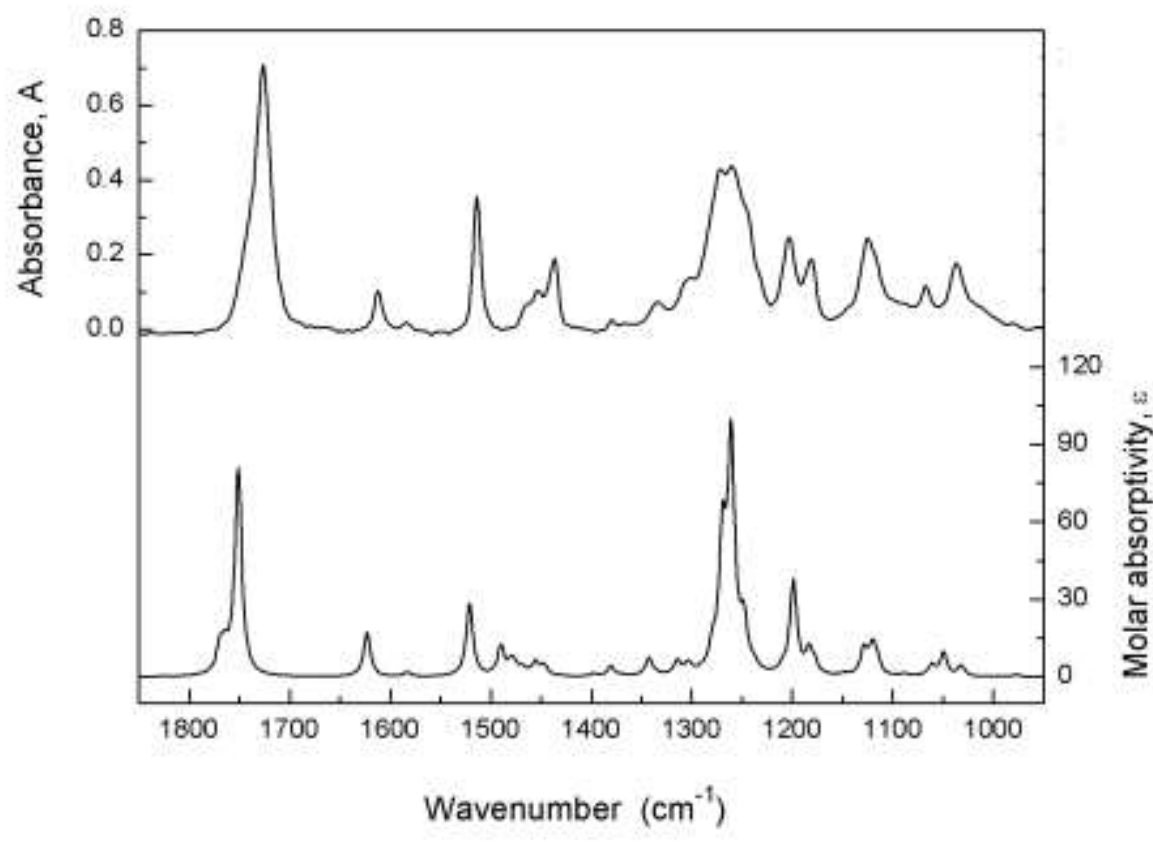

Figure S7 Comparison of the experimental IR spectrum of $\mathbf{3 g}$ (top) and the calculated IR spectra for $(R, R) \mathbf{- 3} \mathbf{g}$ and $(S, S)-\mathbf{3 g}$ (bottom) 


\section{Reference}

S1. Cho, S. J.; Jensen, N. H.; Kurome, T.; Kadari, S.; Manzano, M. L.; Malberg, J. E.; Caldarone, B.; Roth, B. L.; Kozikowski, A. P. J. Med. Chem. 2009, 52, 1885-1902.

S2. (a) Hoye, T. R.; Humpai, P. E.; Moon, B. J. Am. Chem. Soc., 2000, 122, 4982-4983. (b) Pearson; P. J. Org. Chem., 1994 , 59, 5662-5671.

S3. De Keyser, J. -L.; De Cock, C. J. C.; Poupaert, J. H.; Dumont, P. J. Org. Chem. 1988, 53, 4859-4862.

S4. (a) Liao, S.; Sun, X. L.; Tang, Y. Acc. Chem. Res. 2014, 47, 2260-2272. (b) Xiong, H.; Xu, H.; Liao, S.; Xie, Z.; Tang, Y. J. Am. Chem. Soc. 2013, 135, 7851-7854. (c) Liu, Q. J.; Yan, W. -G.; Wang, L. J.; Zhang, X. P.; Tang, Y. Org. Lett. 2015, 17, 4014-4017. 


\section{8. ${ }^{1} \mathrm{H}$ NMR and ${ }^{13} \mathrm{C}$ NMR Spectra of Compounds}

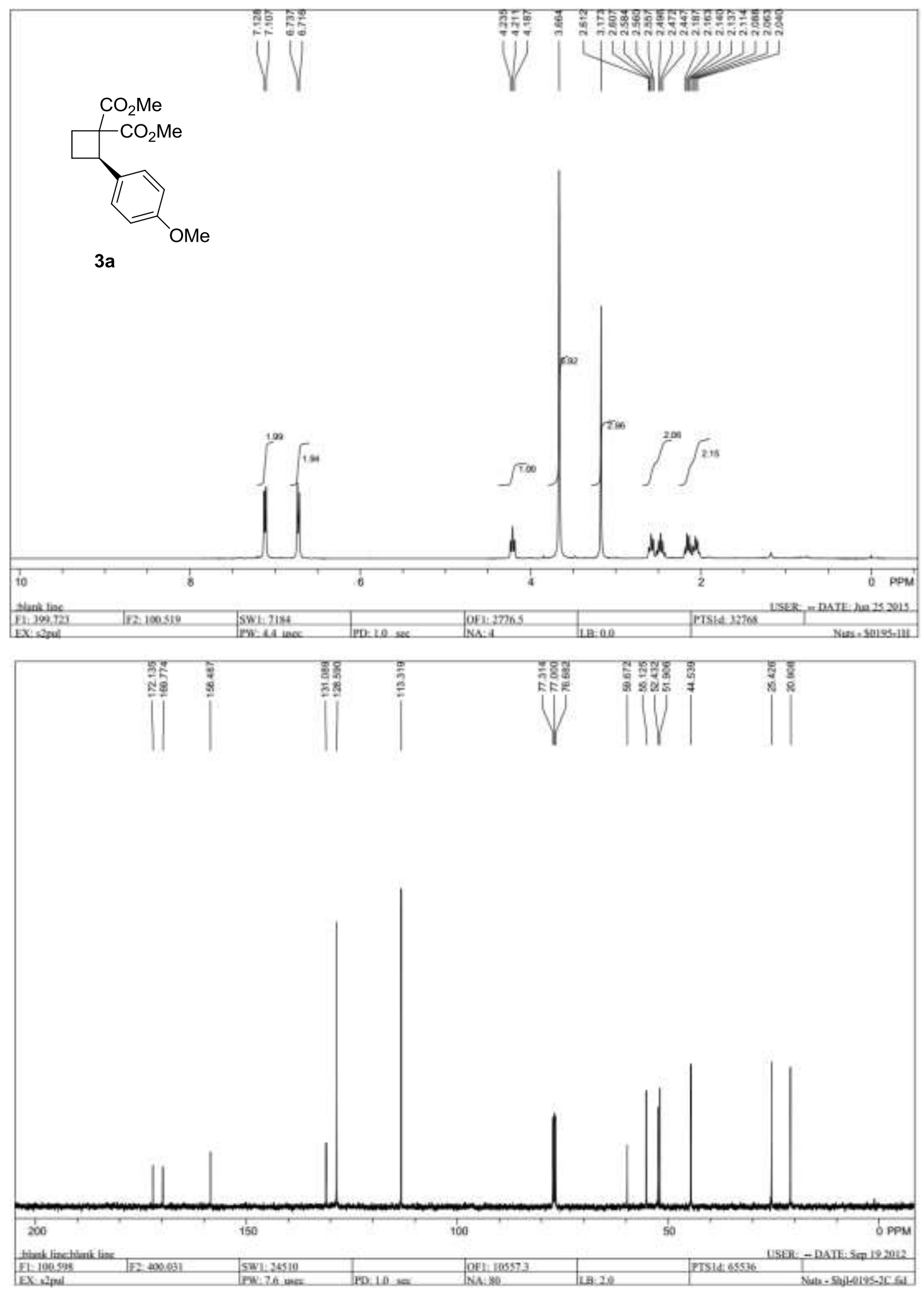




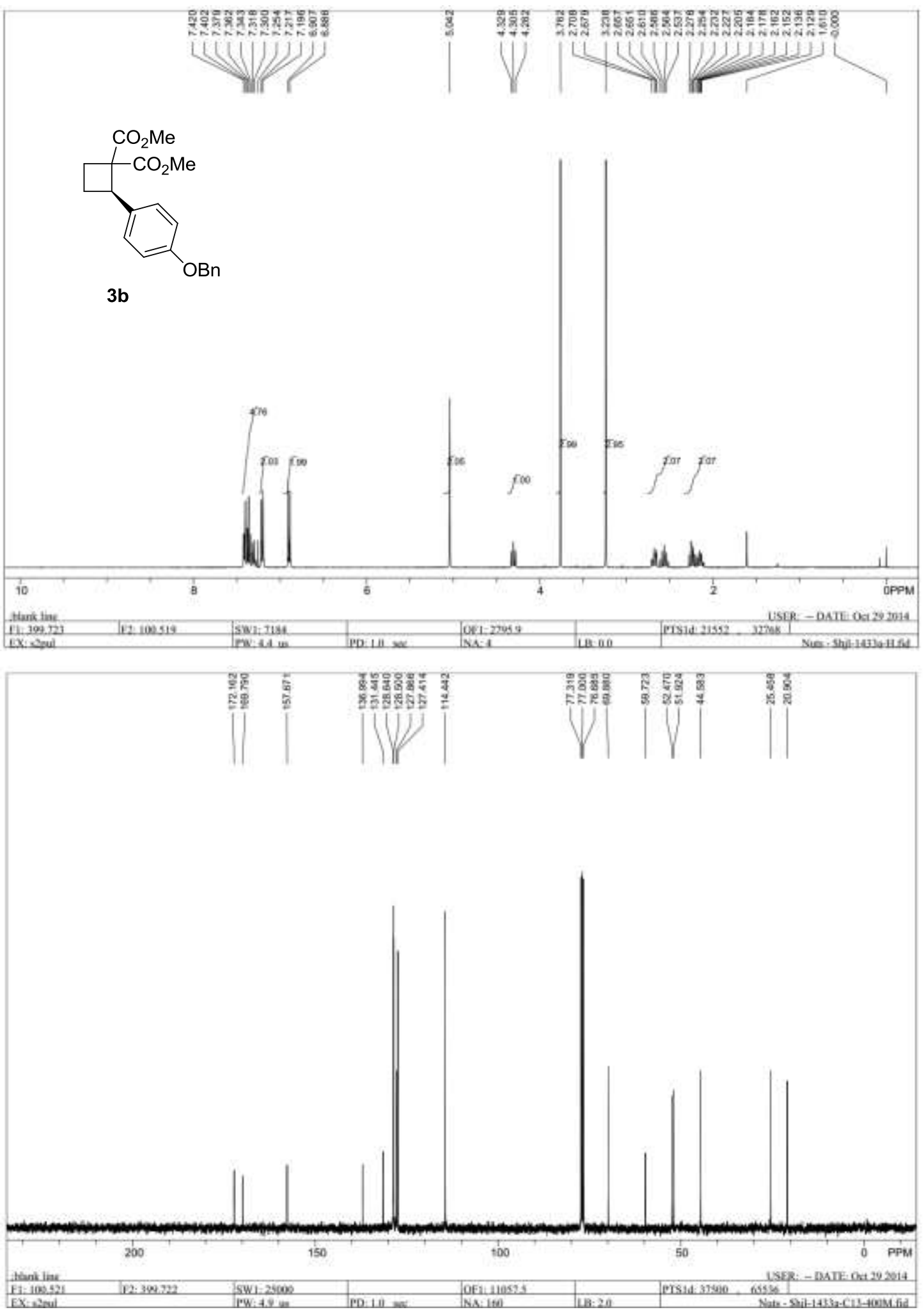



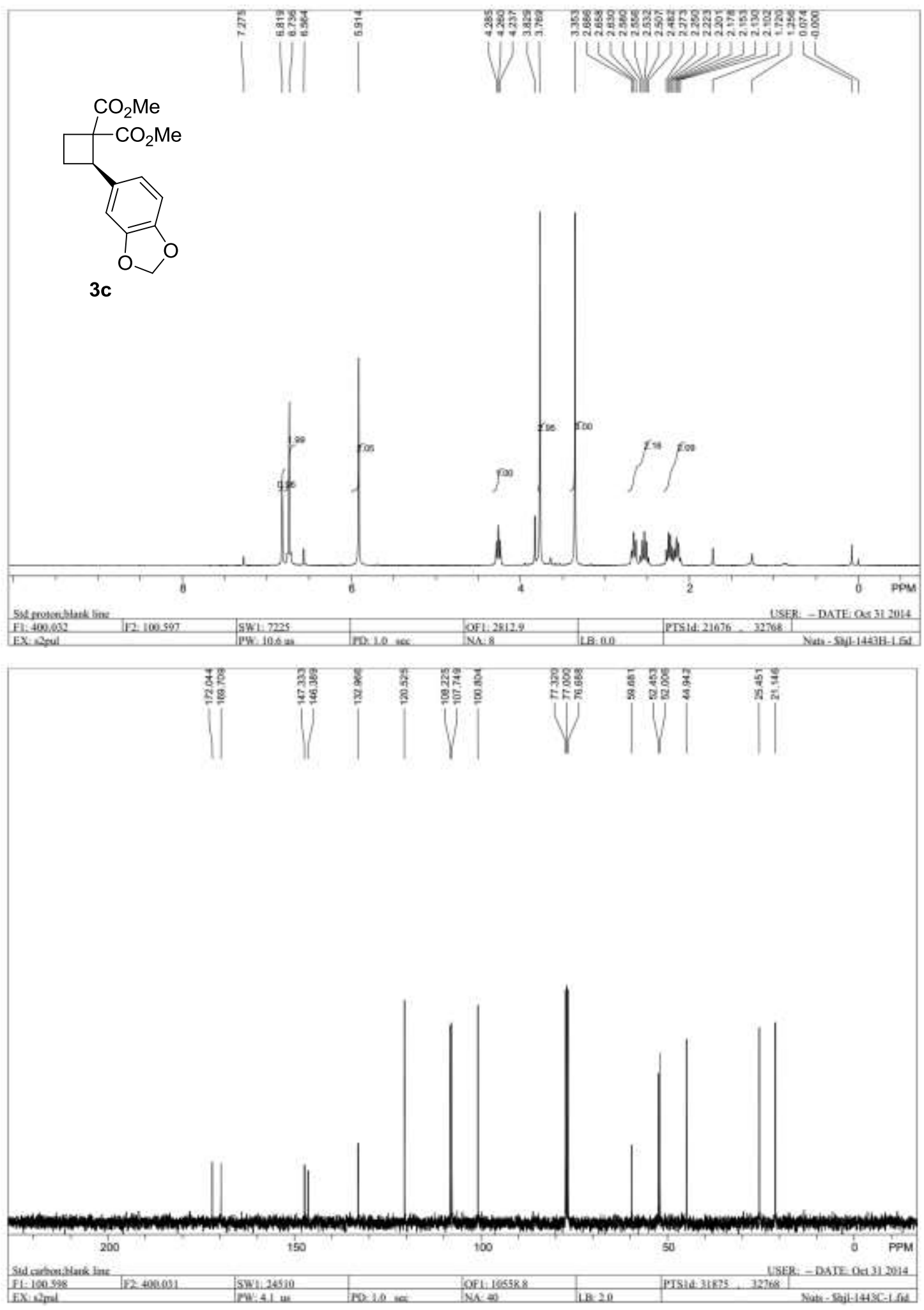

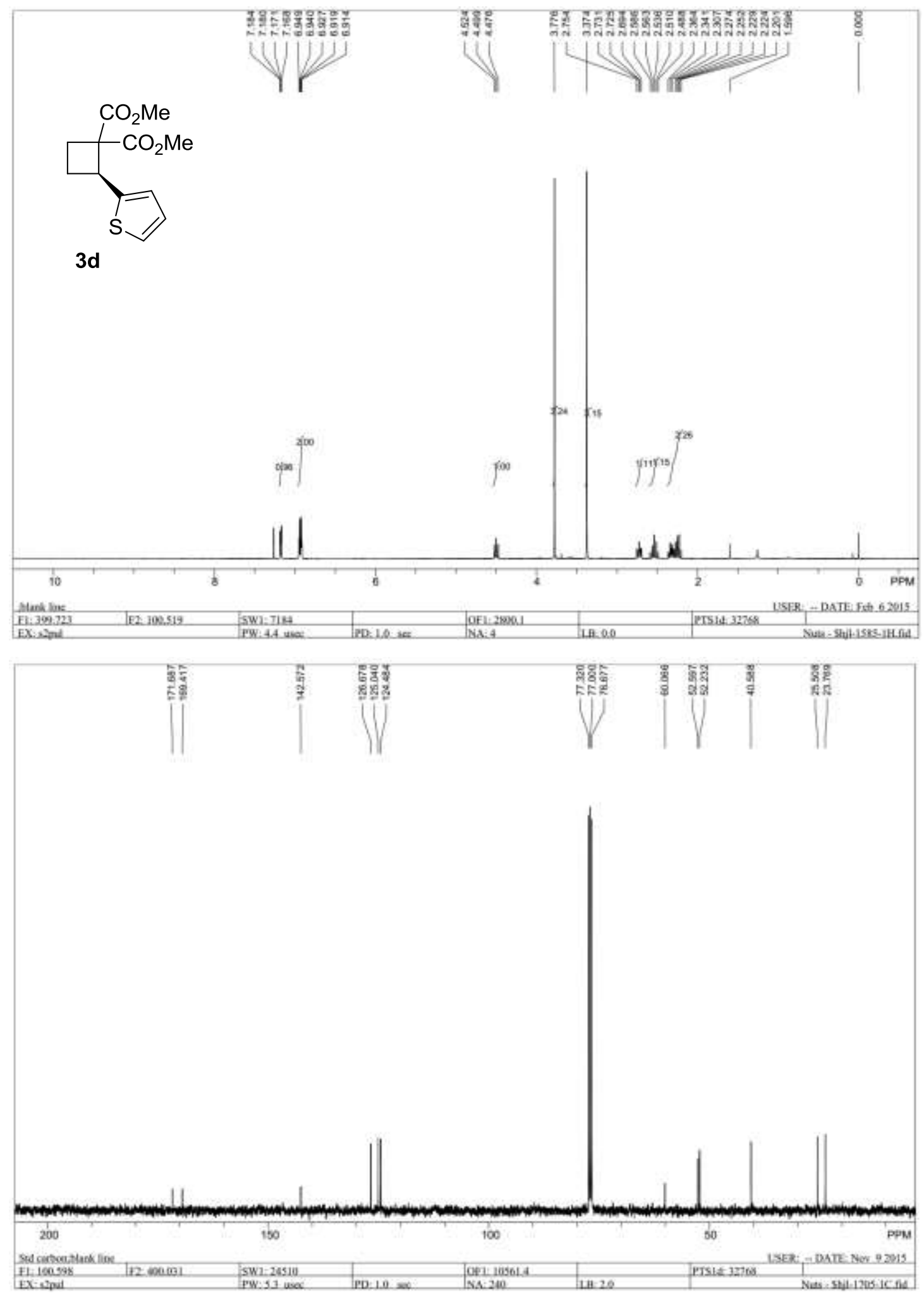


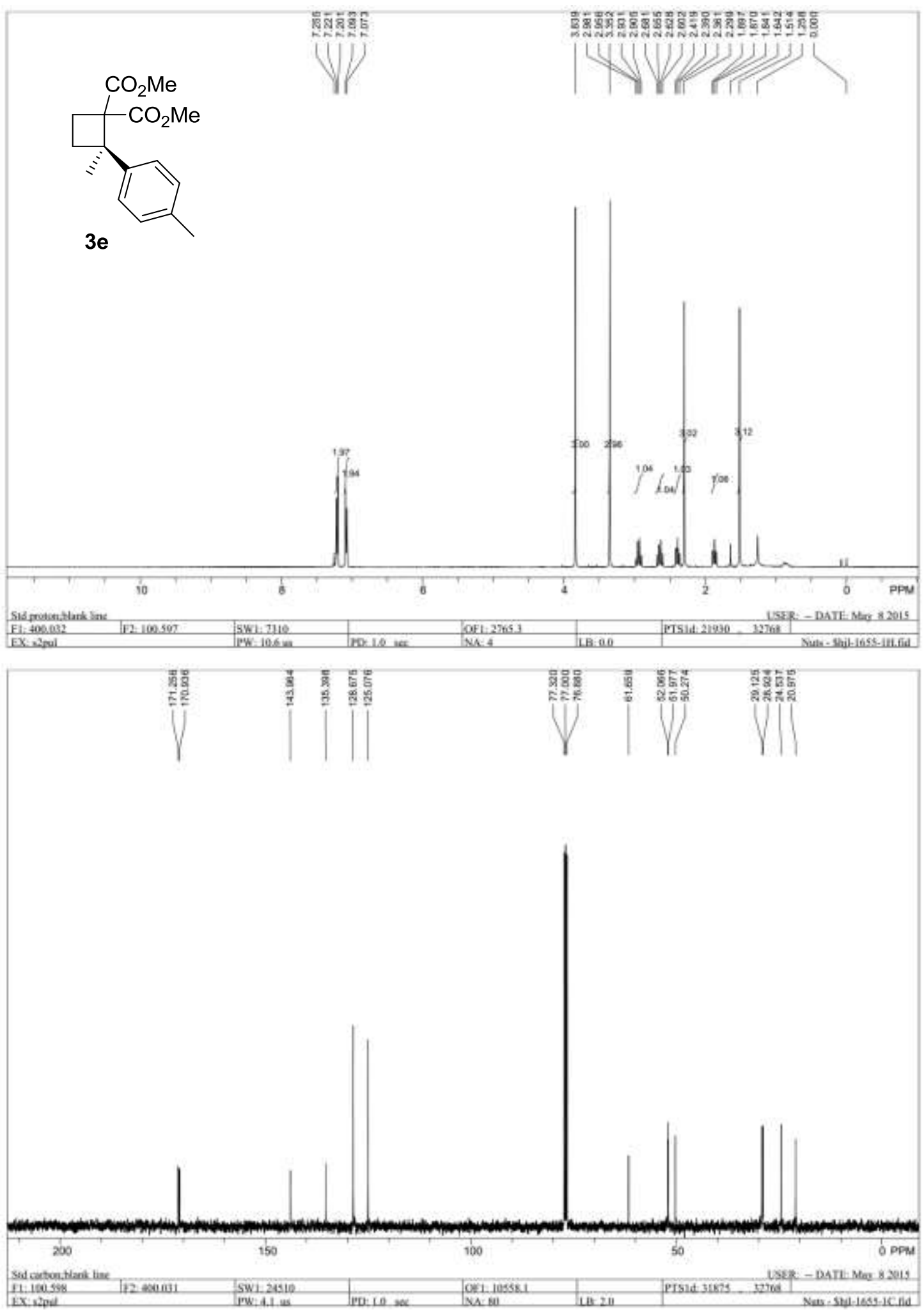



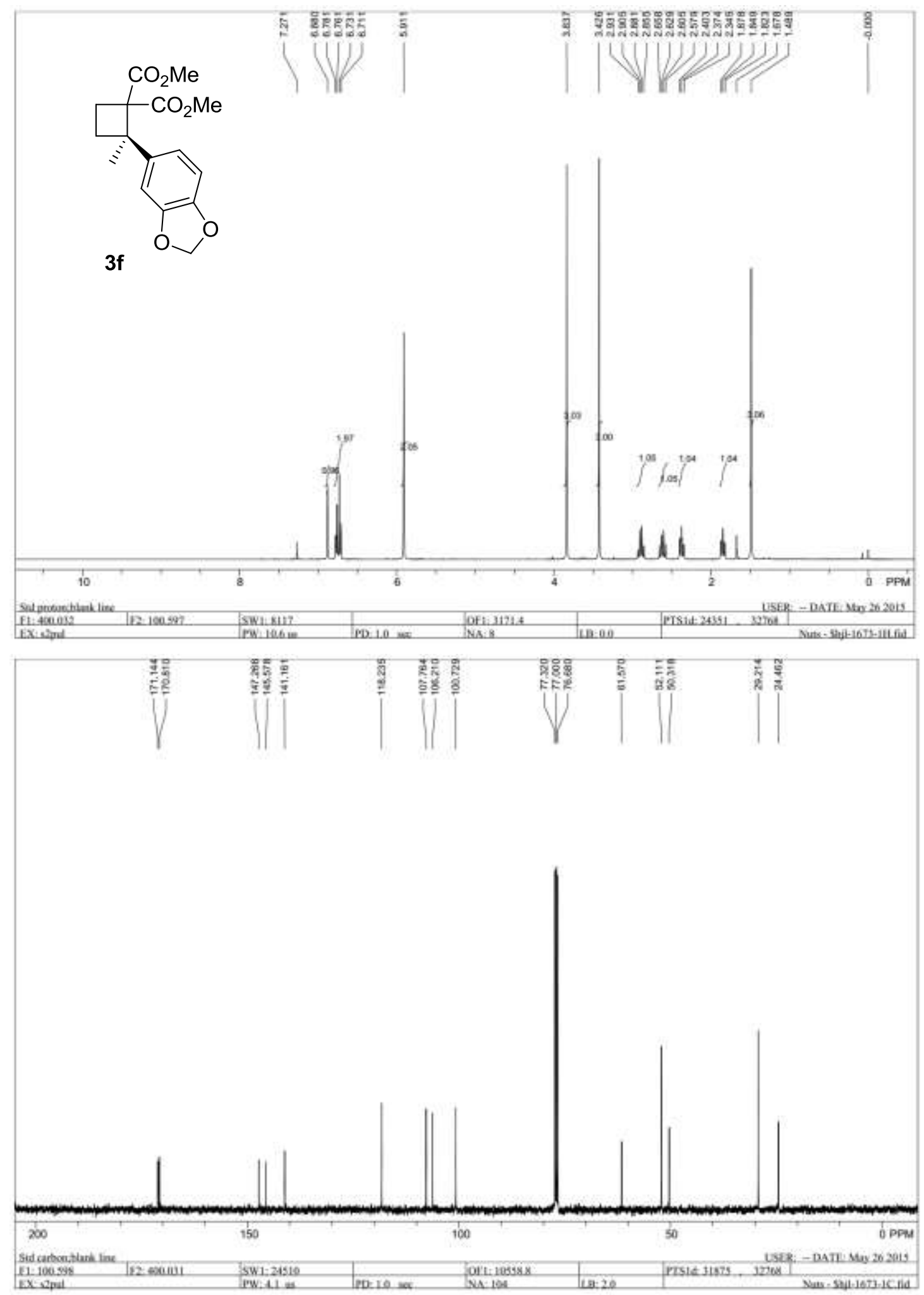


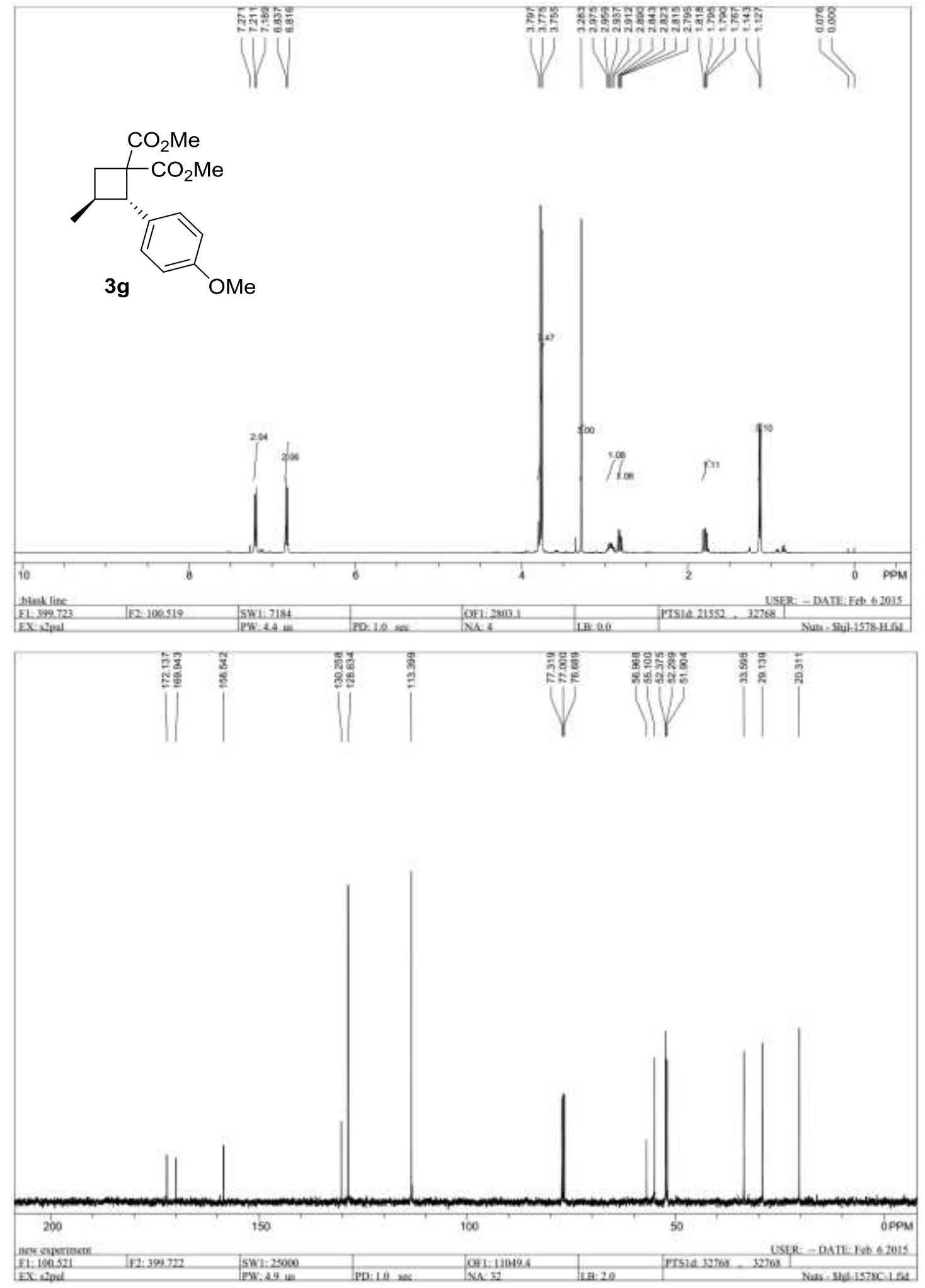



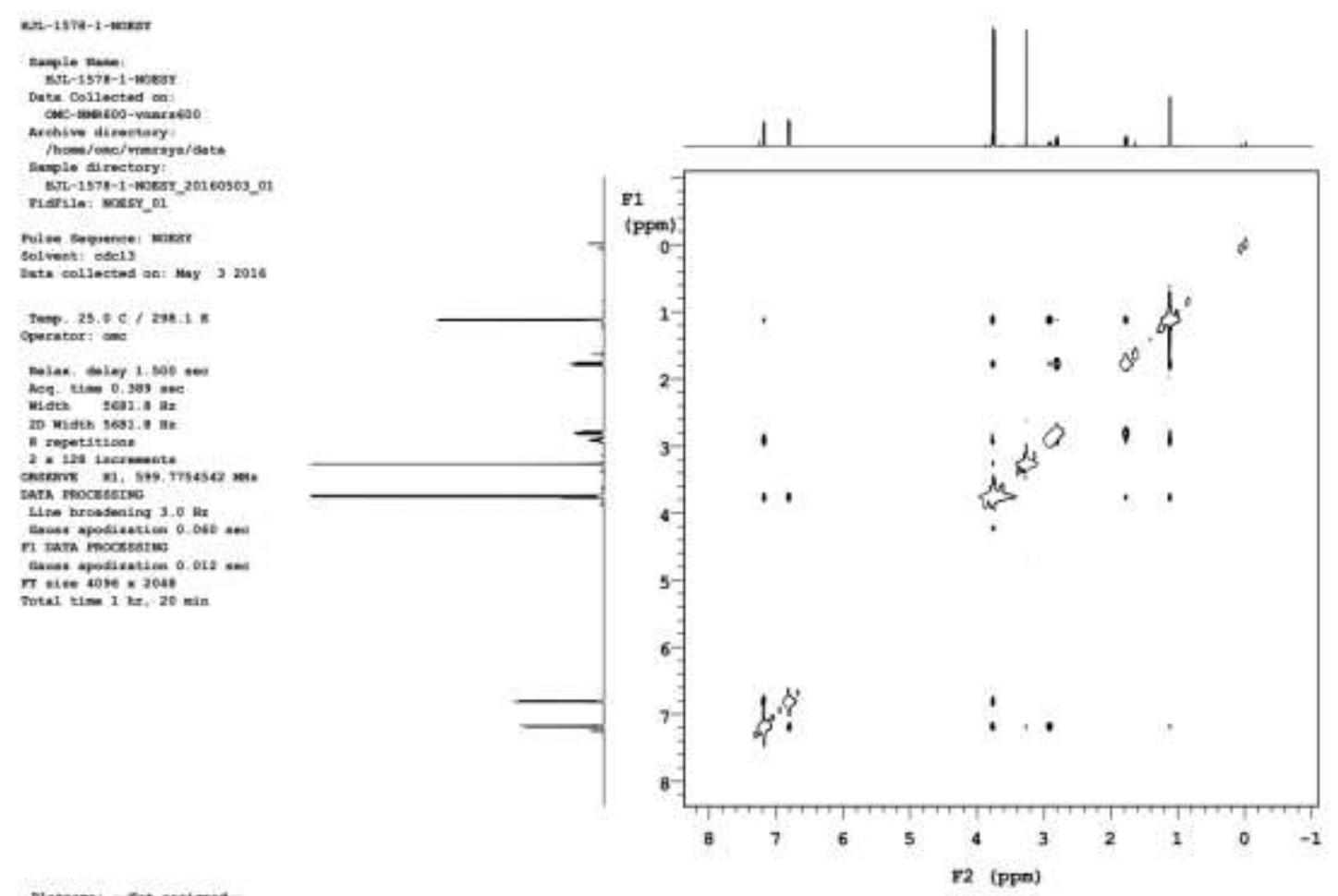

Dlotame - Sot antigned-

ar-1970-1-poser

Imple now:

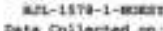

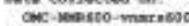

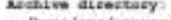

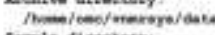

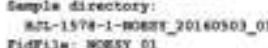

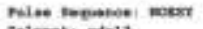

hol want: ederis

Dats oollestad on: May I zoue

ogeratos: $29.90 / 296.1$.

Nelex detay 1,500 wec

vide

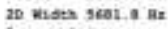

i caperieticas.

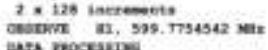

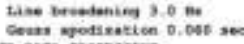

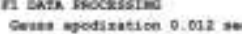

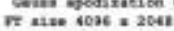

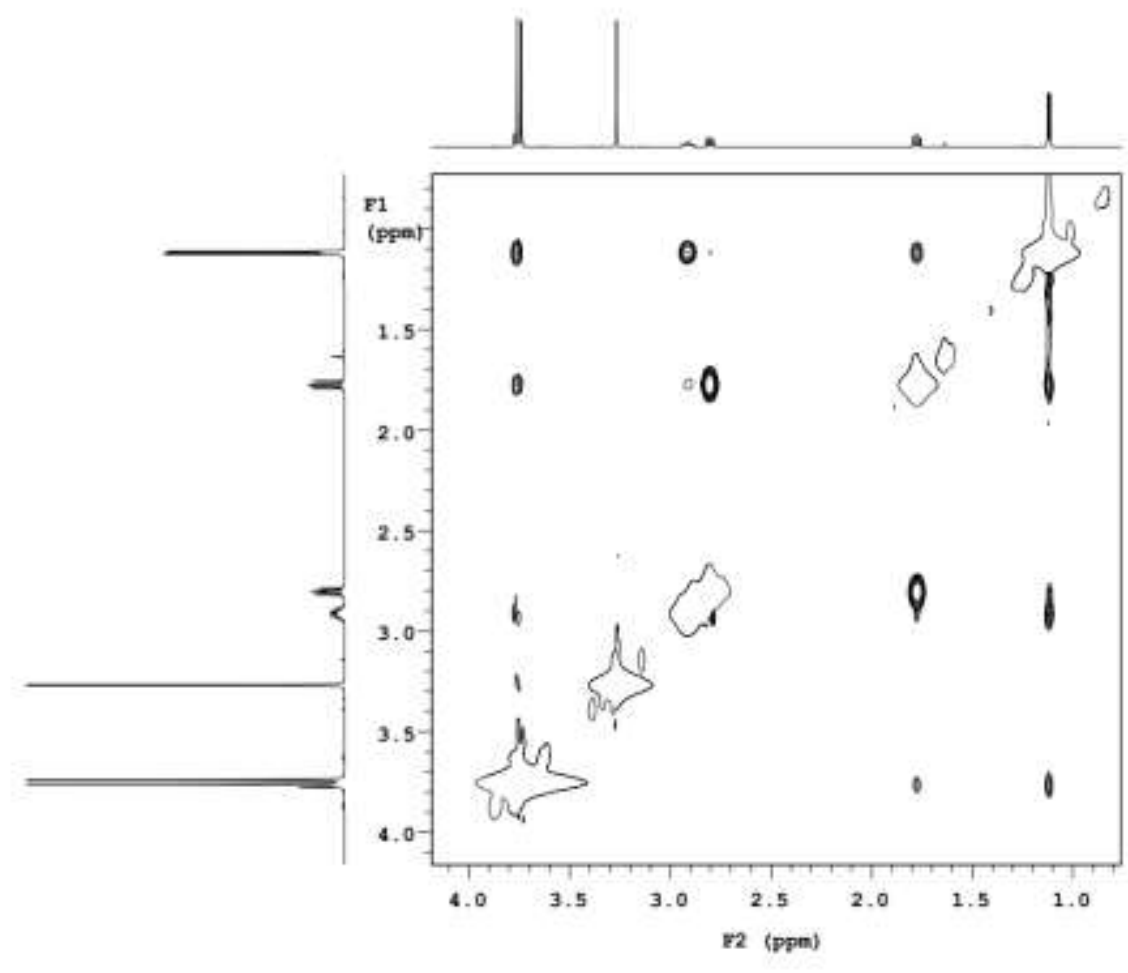

Vetnuse: -Hot anrighed- 
an-2s) -1-ketar

Smegion the

24-157-1-veses

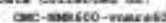

nosive disesteey.

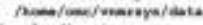

qia Airotery

arb-197i-1-

narile: coestas

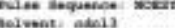

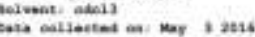

nev. as a c / $206 . t \mathrm{~K}$

Nolise. onley 1, 500 ope

Not. $\operatorname{tin} 0.319300$

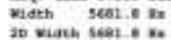

20 viath sait. . .

tores tom

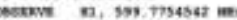

The mockistas

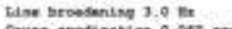

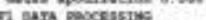

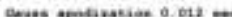

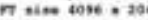

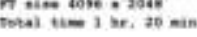

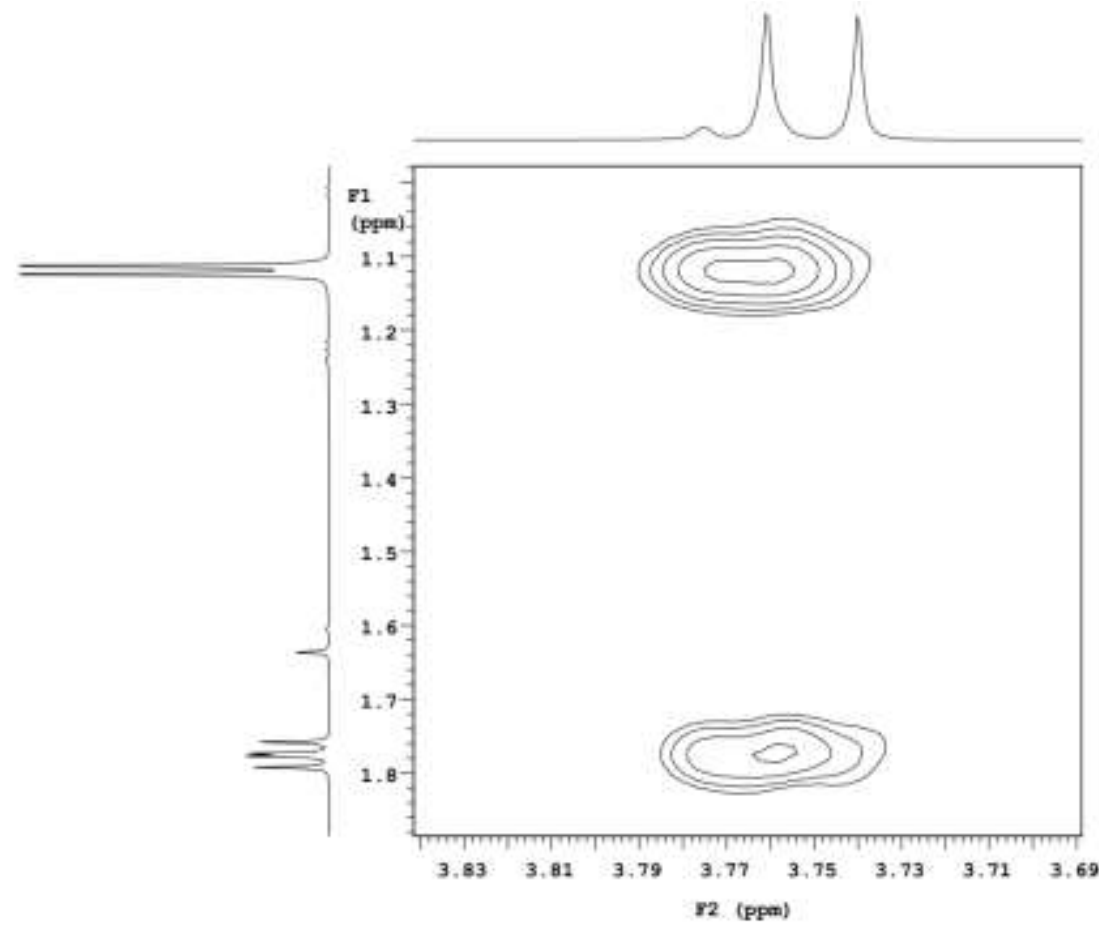

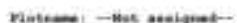




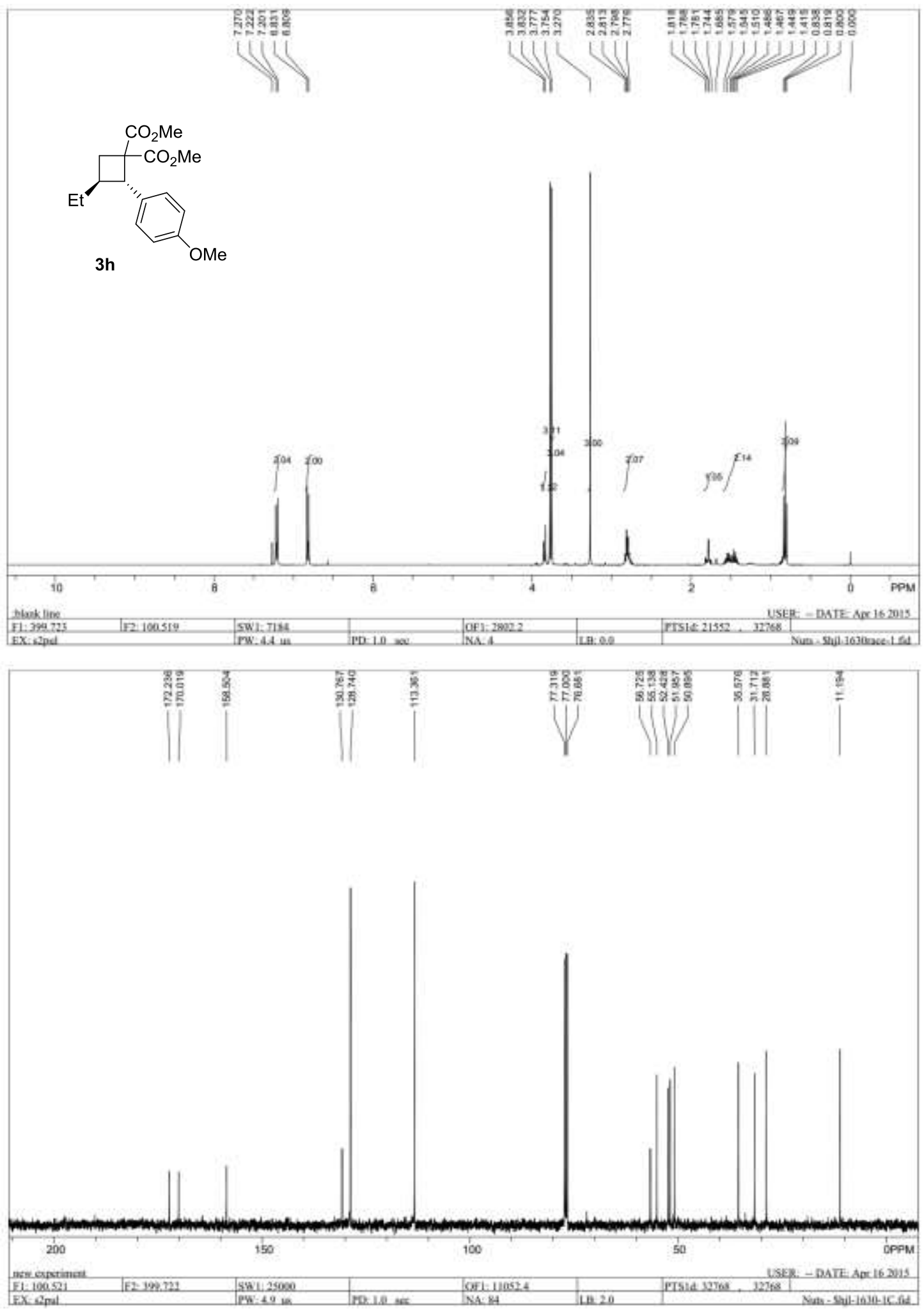




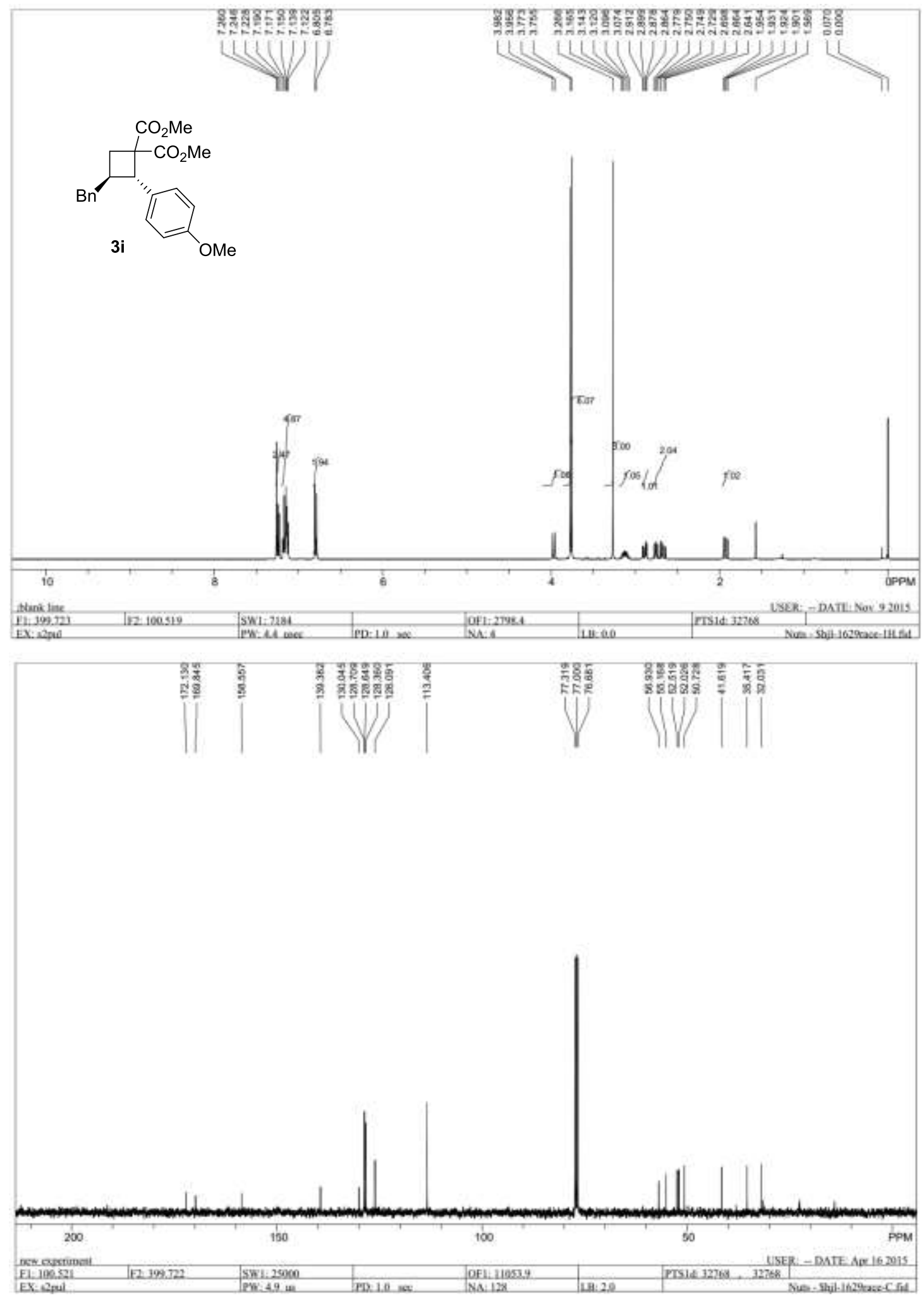




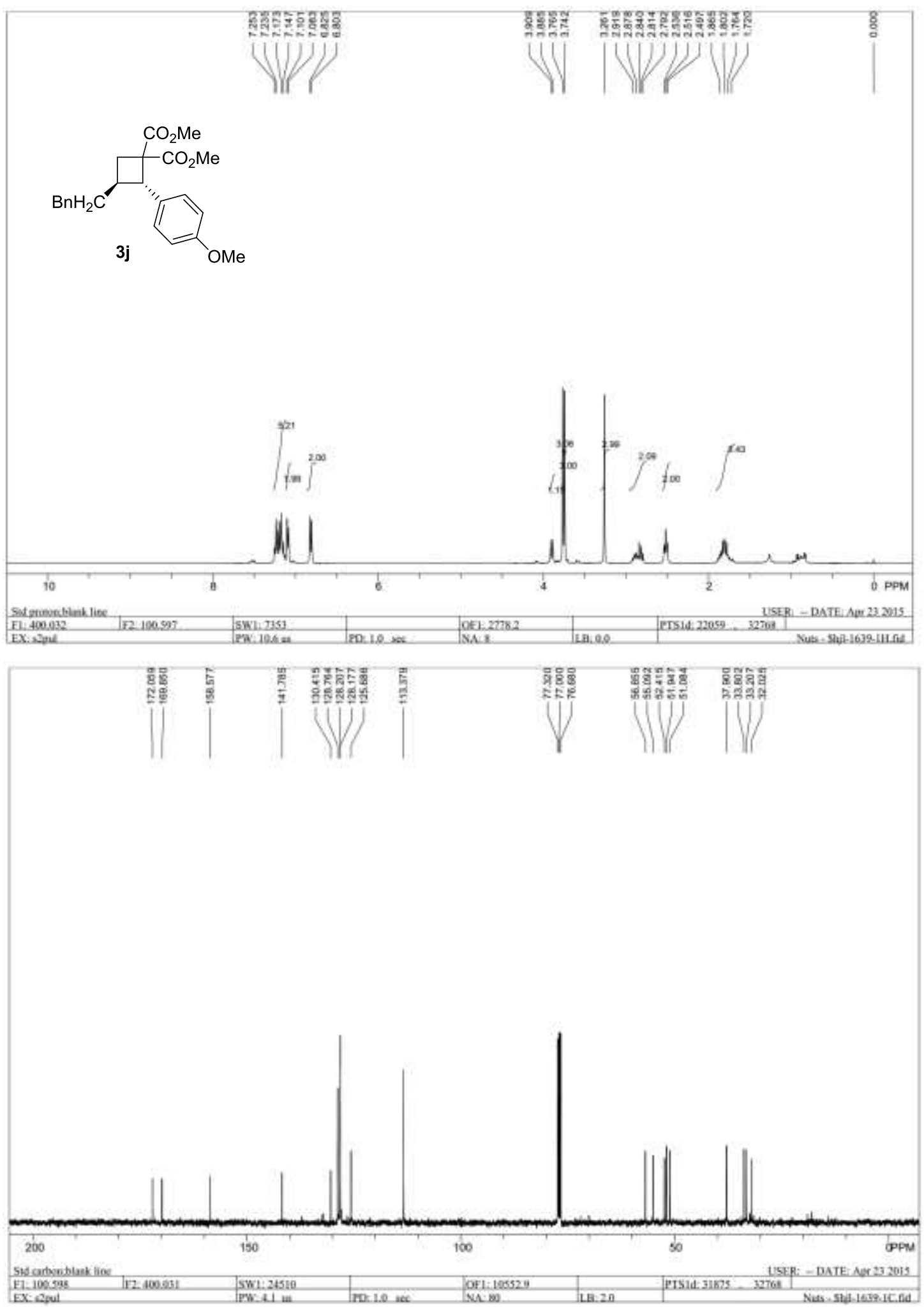




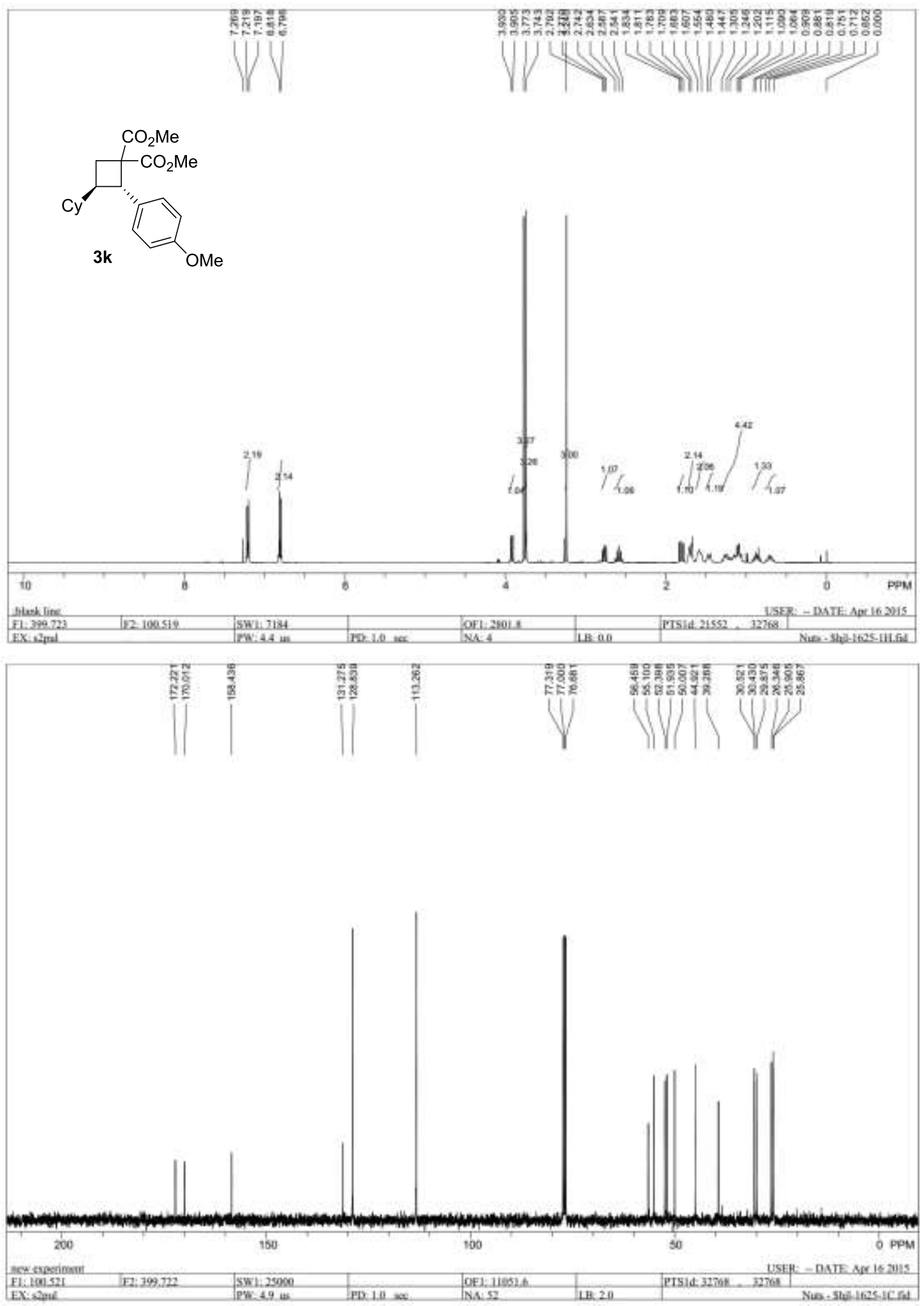




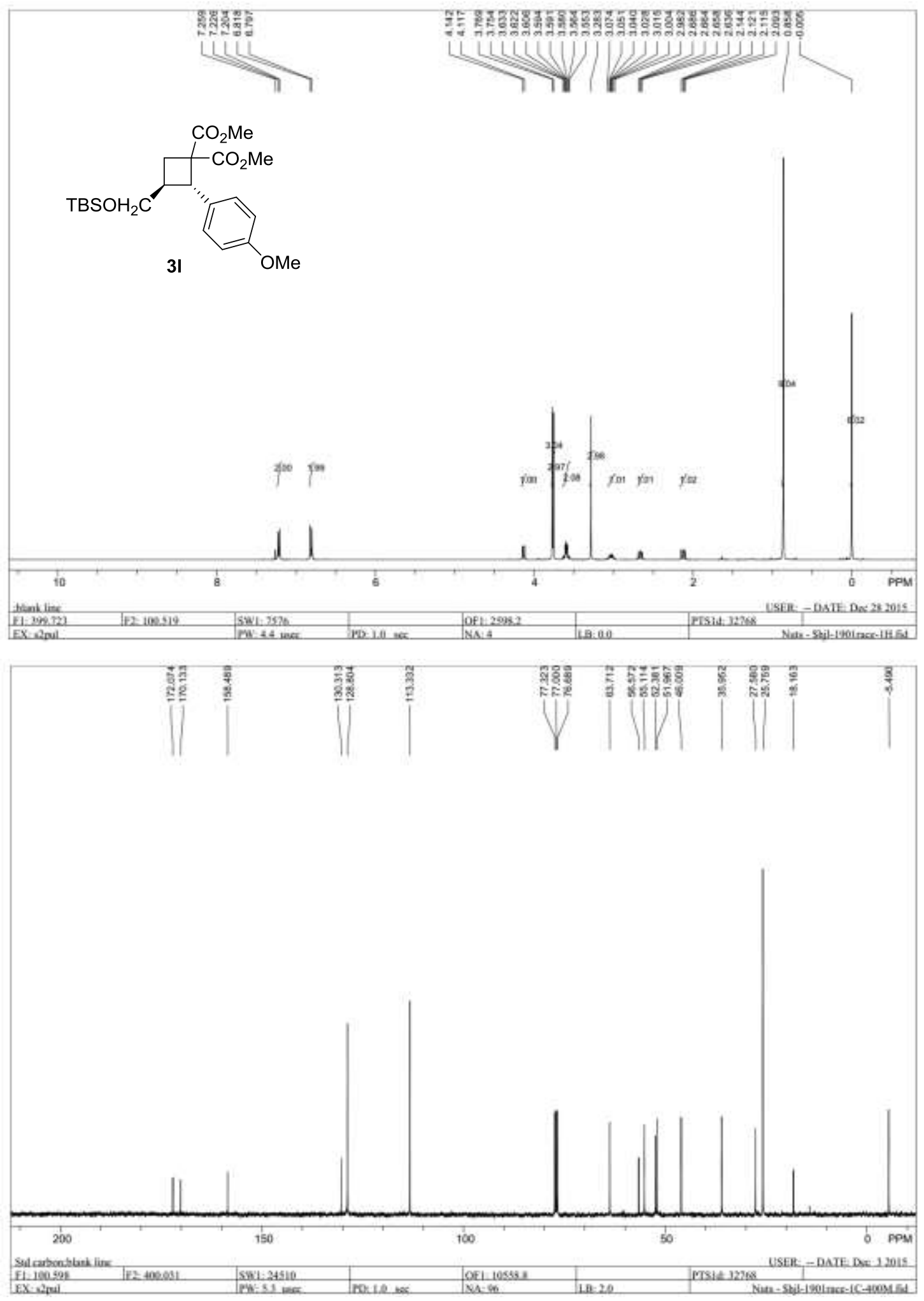




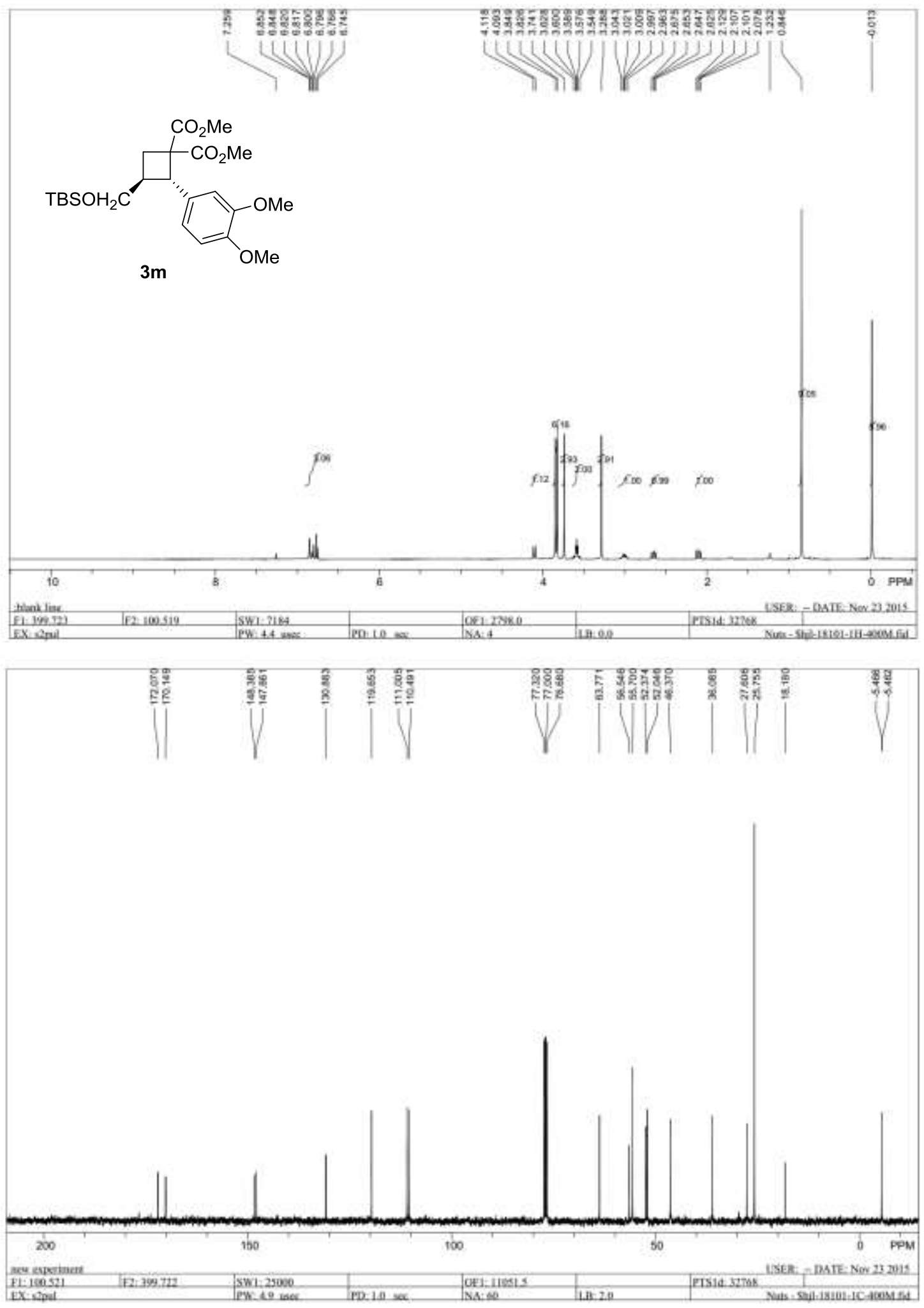



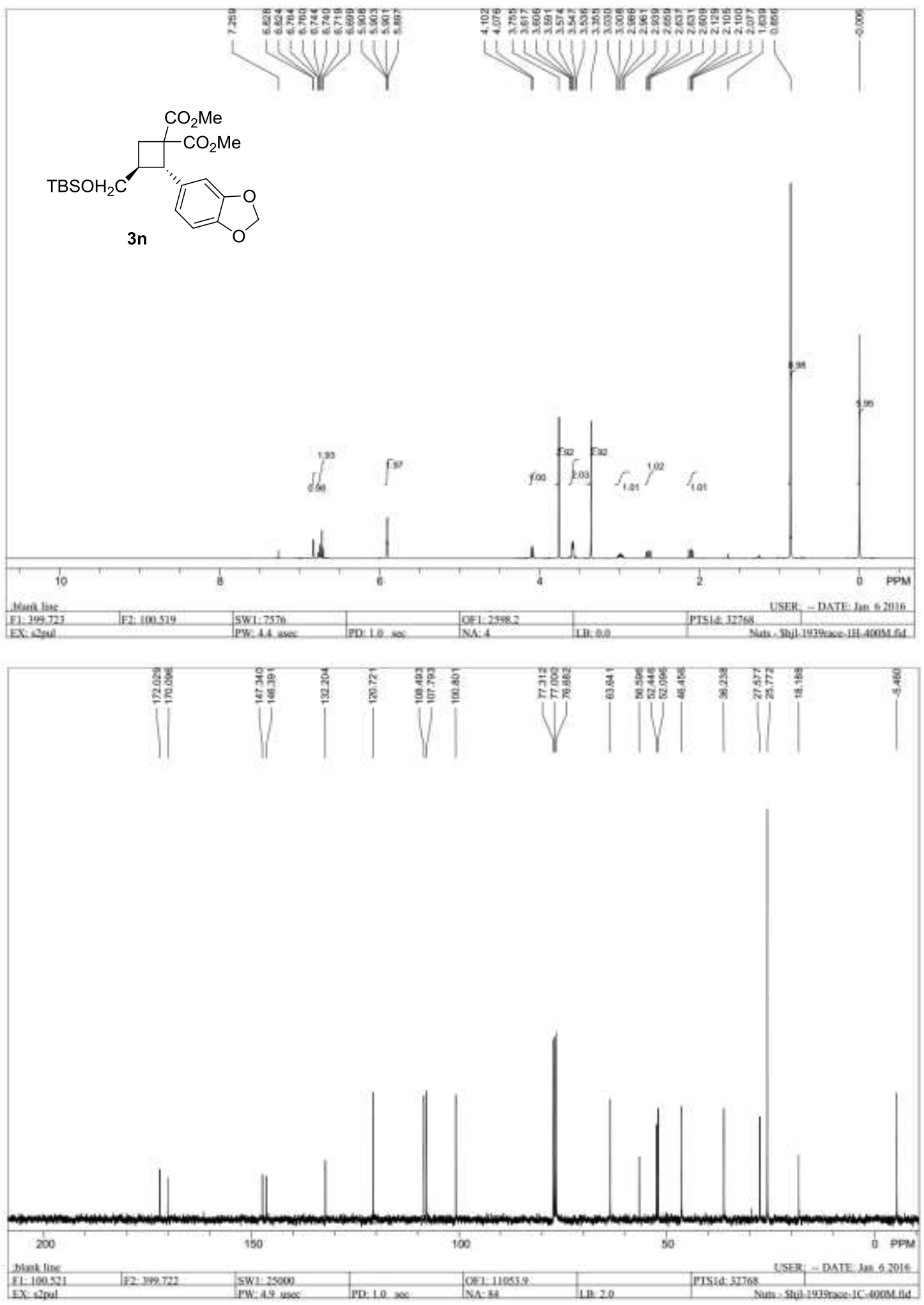

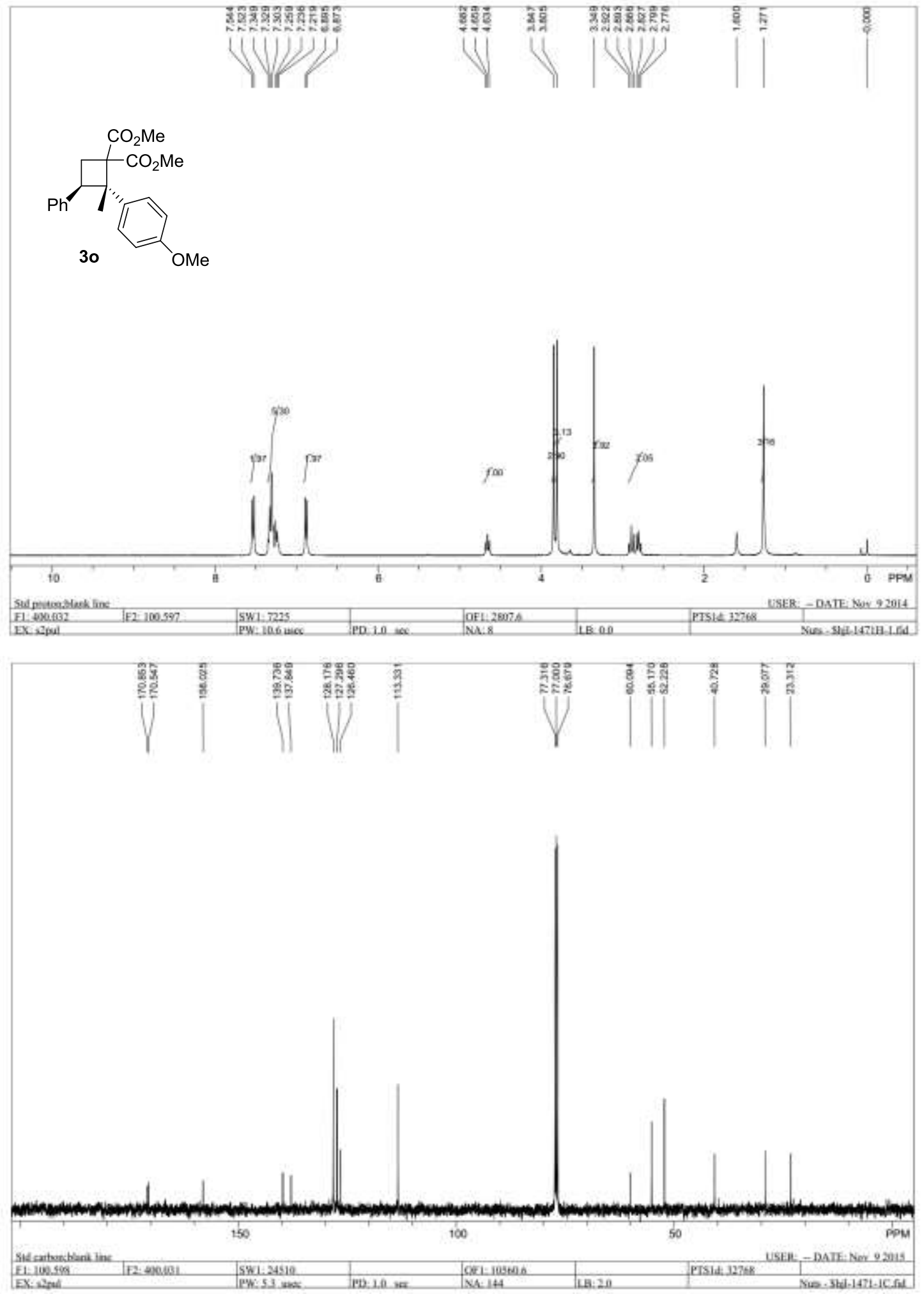

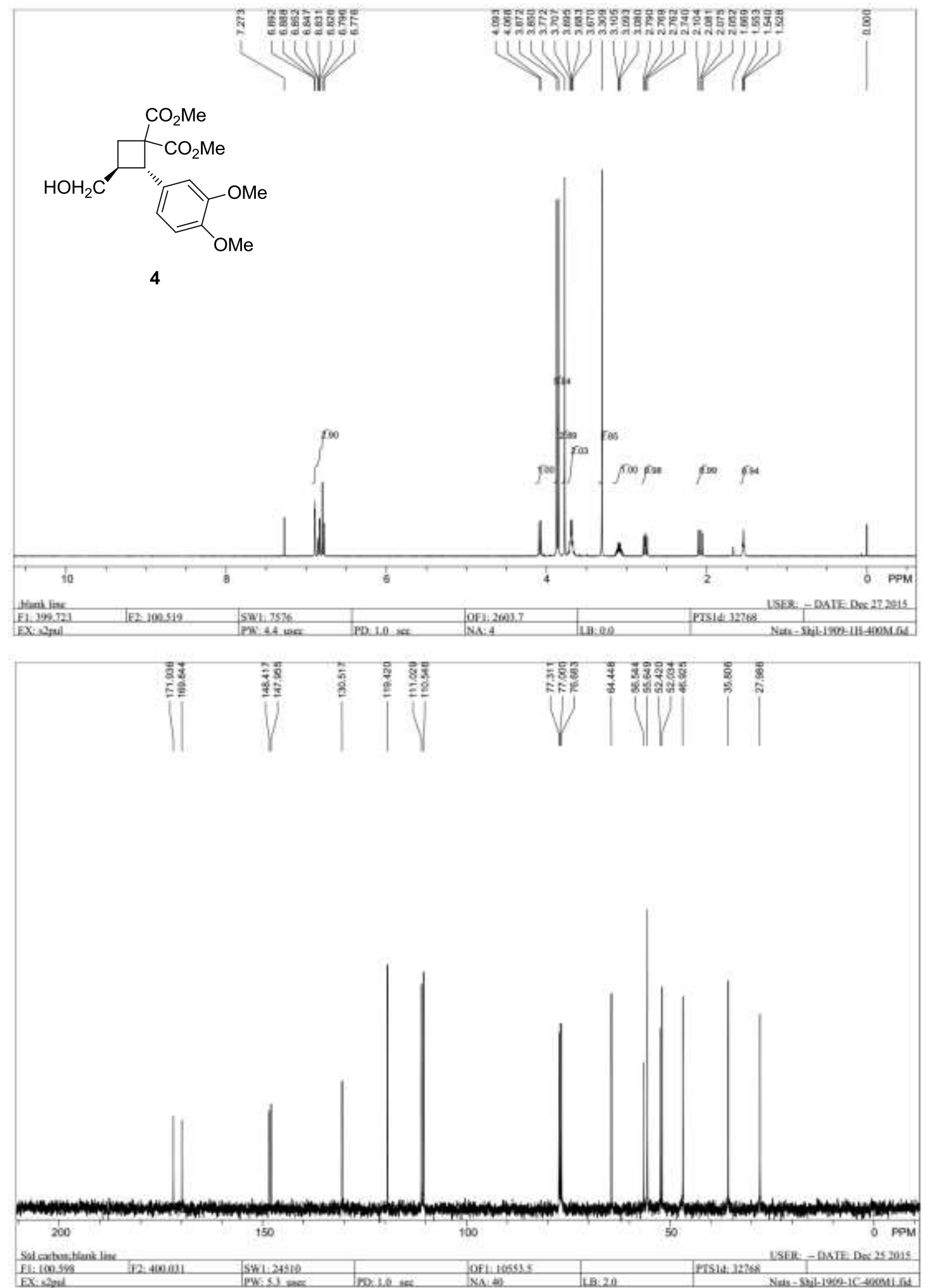

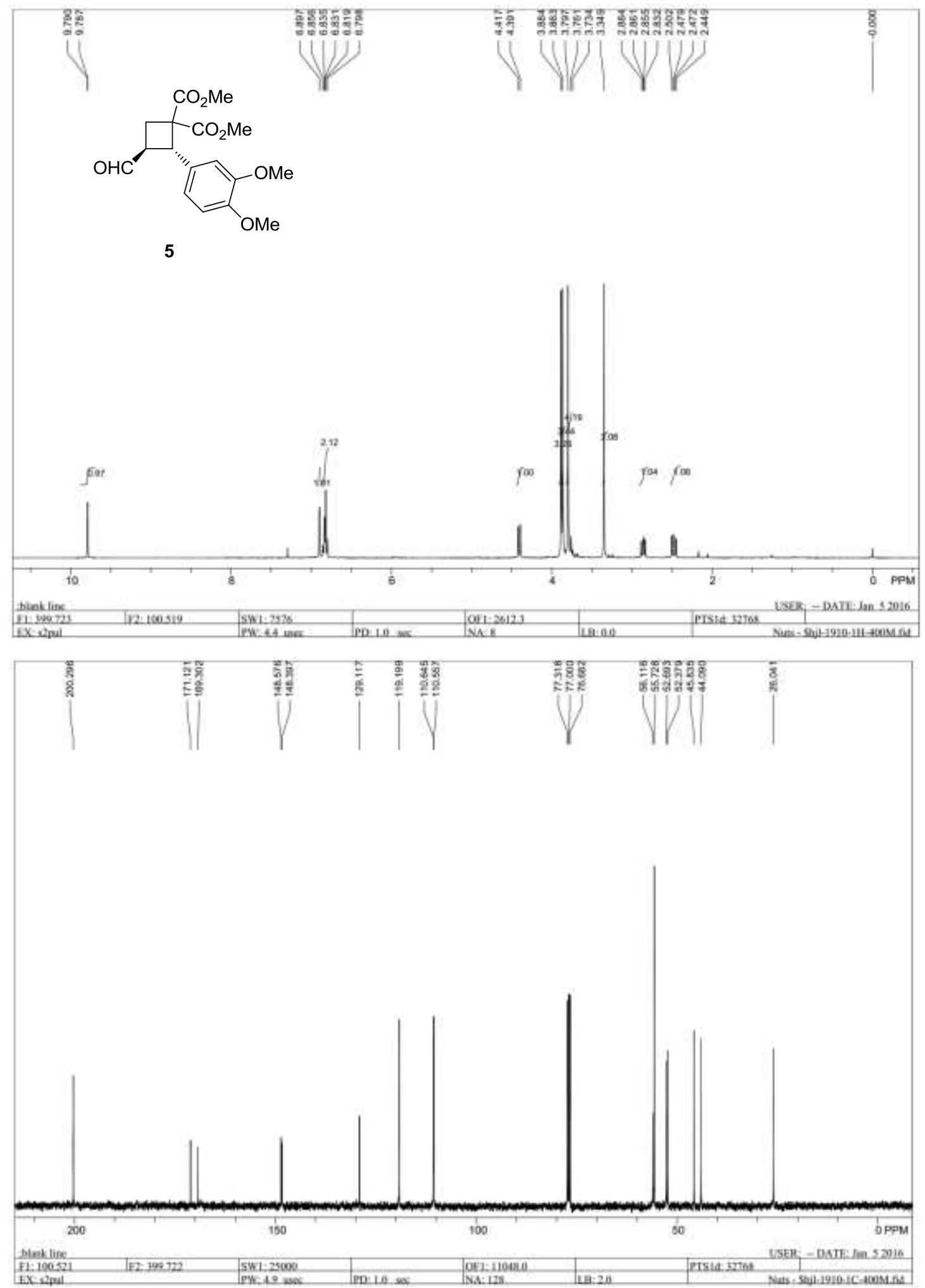


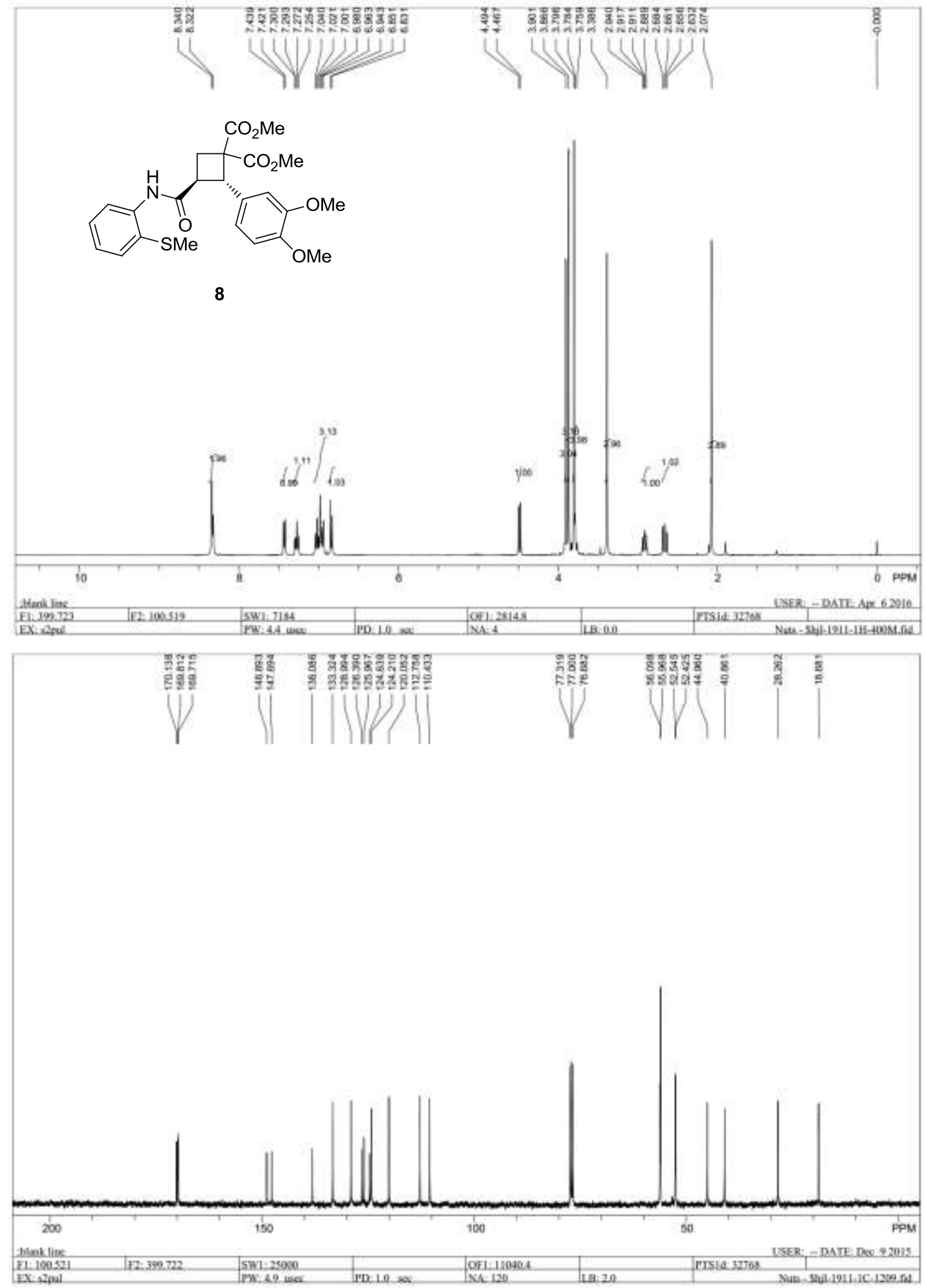



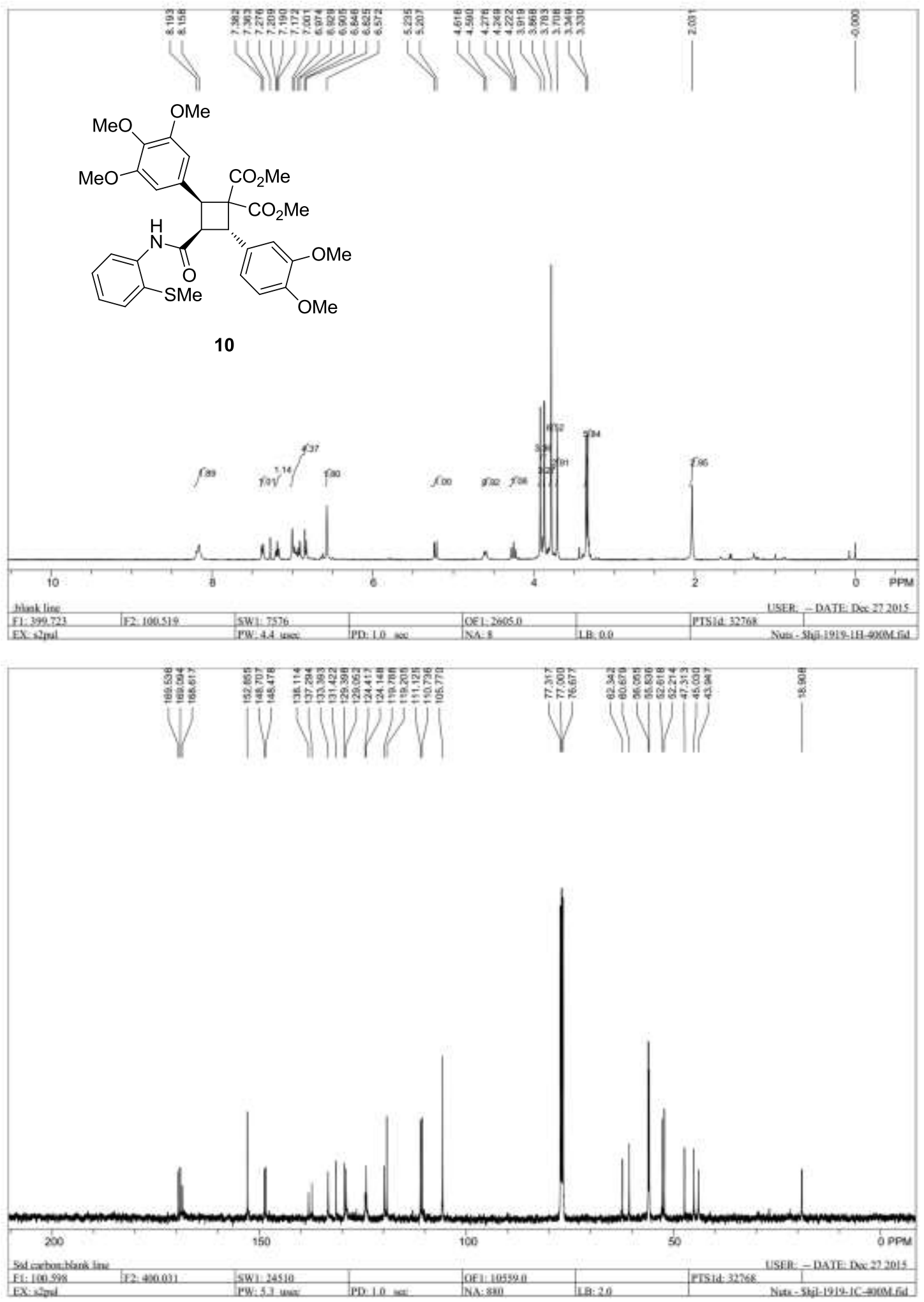

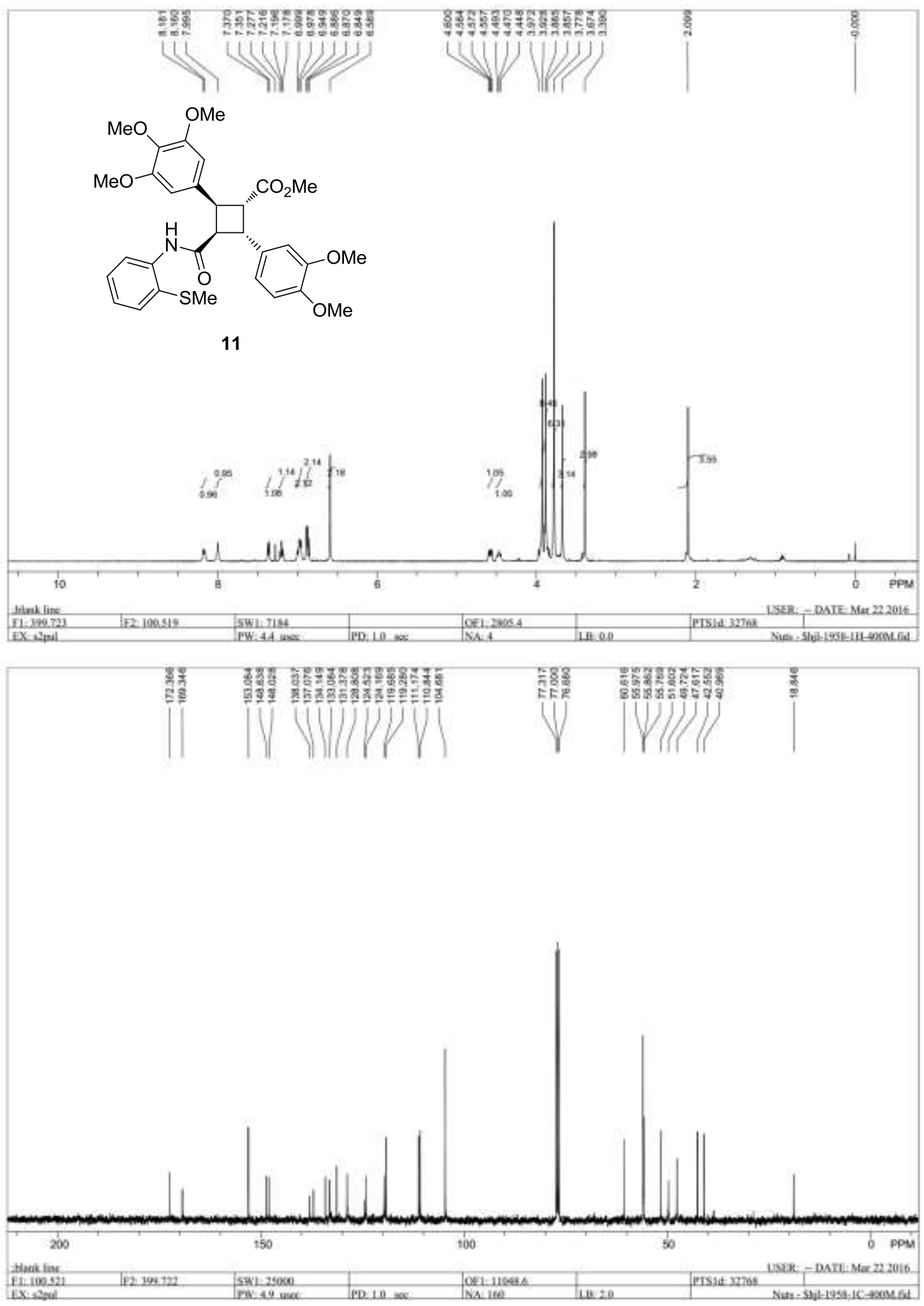

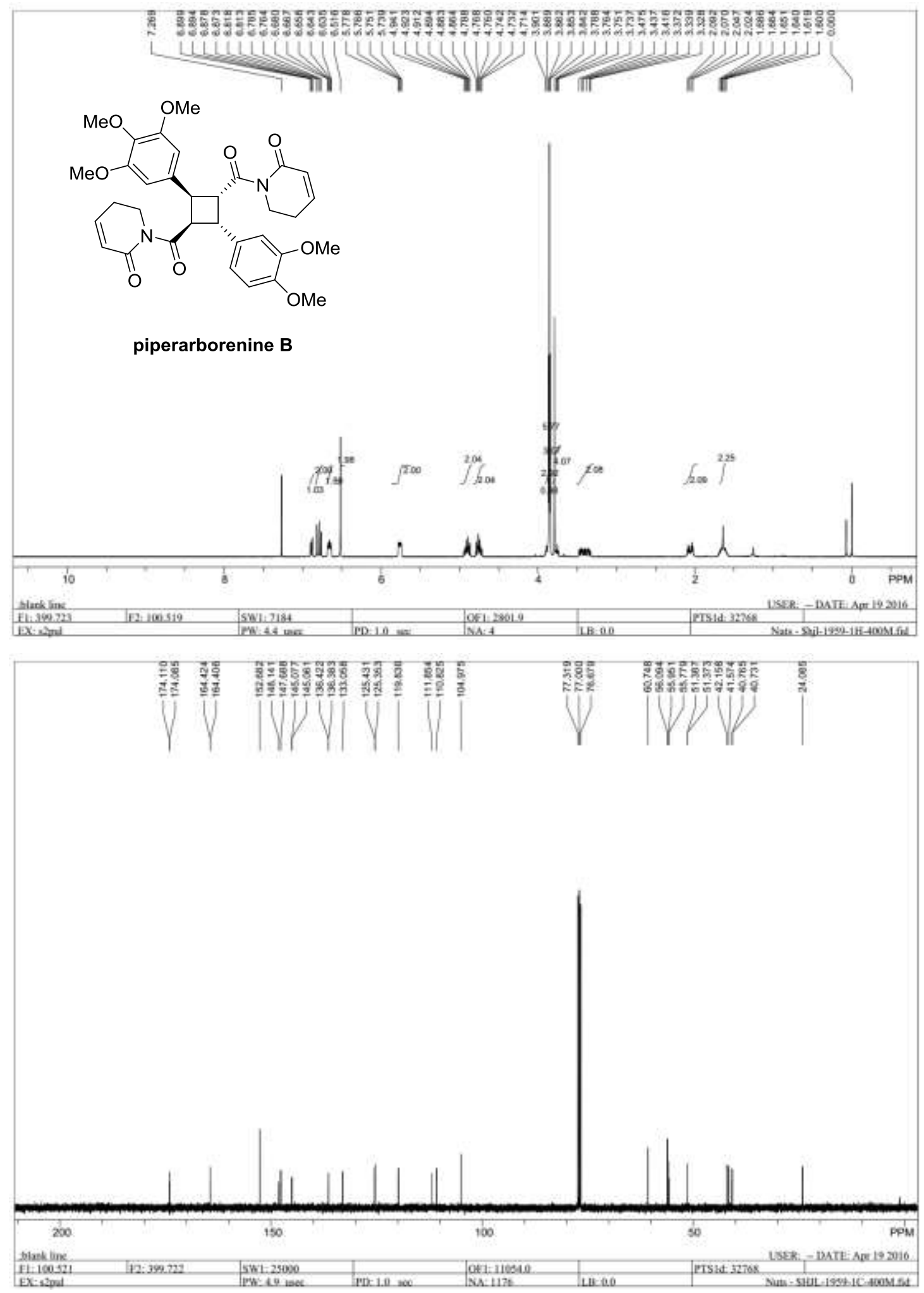


\section{HPLC Spectra of Compounds}

<Chromatogram>

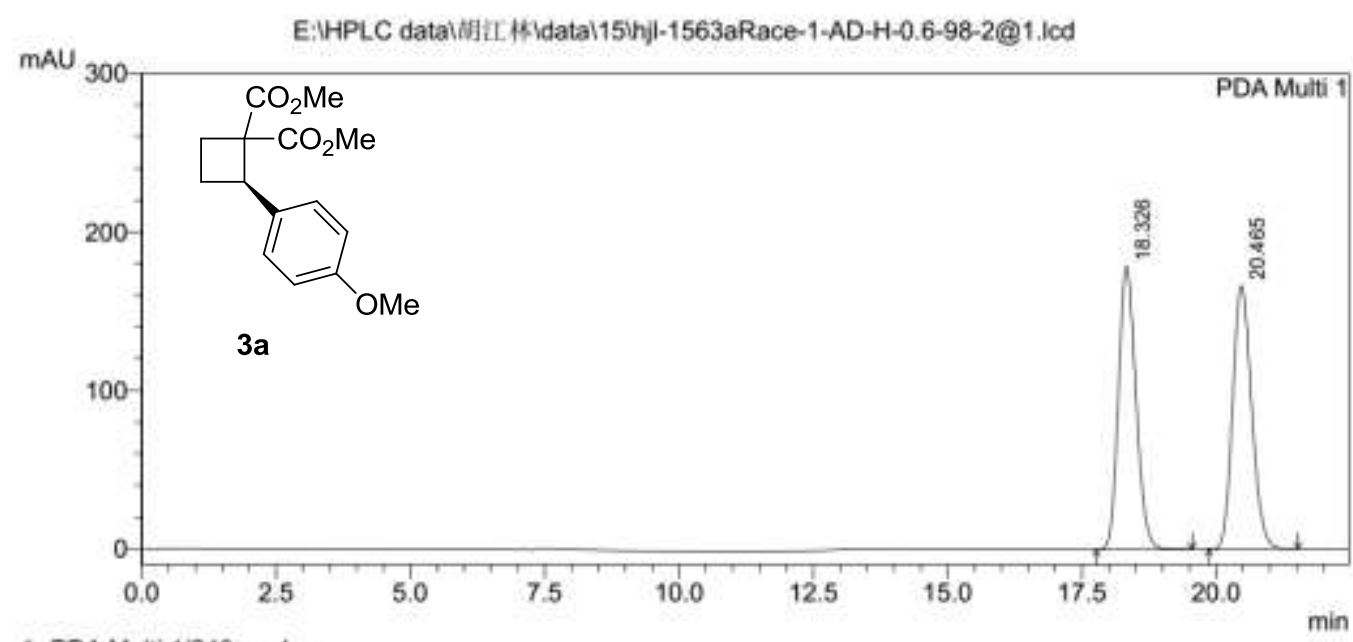

1 PDA Multi $1 / 240 \mathrm{~nm} 4 \mathrm{~nm}$

<Results>

PDA Ch1 240nm 4nm
\begin{tabular}{|l|r|r|r|r|r|r|}
\hline Peak \# & Ret. Time & Area & Height & Area $\%$ & Height \% & Mark \\
\hline 1 & 18.326 & 4047852 & 178571 & 49.797 & 51.781 & \\
\hline 2 & 20.465 & 4080791 & 166287 & 50.203 & 48.219 & \\
\hline Total & & 8128643 & 344858 & 100.000 & 100.000 & \\
\hline
\end{tabular}

\section{$<$ Chromatogram>}

E:HPLC datal胡江 林idatal15ihjl-1563a-AD-H-0.6-98-2@4.Iod

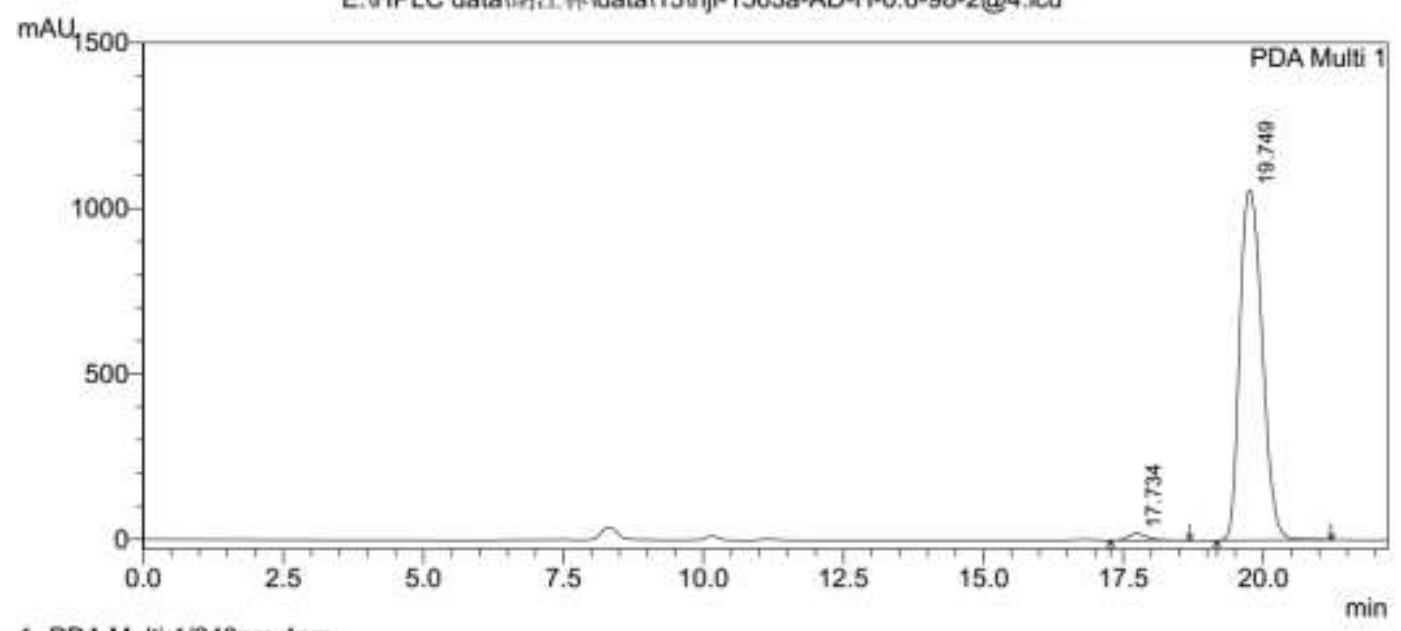

1 PDA Multi $1 / 240 \mathrm{~nm} 4 \mathrm{~nm}$

\section{$<$ Results>}

PDA Ch1 240 nm 4nm
\begin{tabular}{|l|r|r|r|r|r|r|}
\hline Peak \# & Ret. Time & Area & Height & Area $\%$ & Height \% & Mark \\
\hline 1 & 17.734 & 460009 & 20299 & 1.546 & 1.882 & \\
\hline 2 & 19.749 & 29288712 & 1058453 & 98.454 & 98.118 & \\
\hline Total & & 29748720 & 1078752 & 100.000 & 100.000 & \\
\hline
\end{tabular}




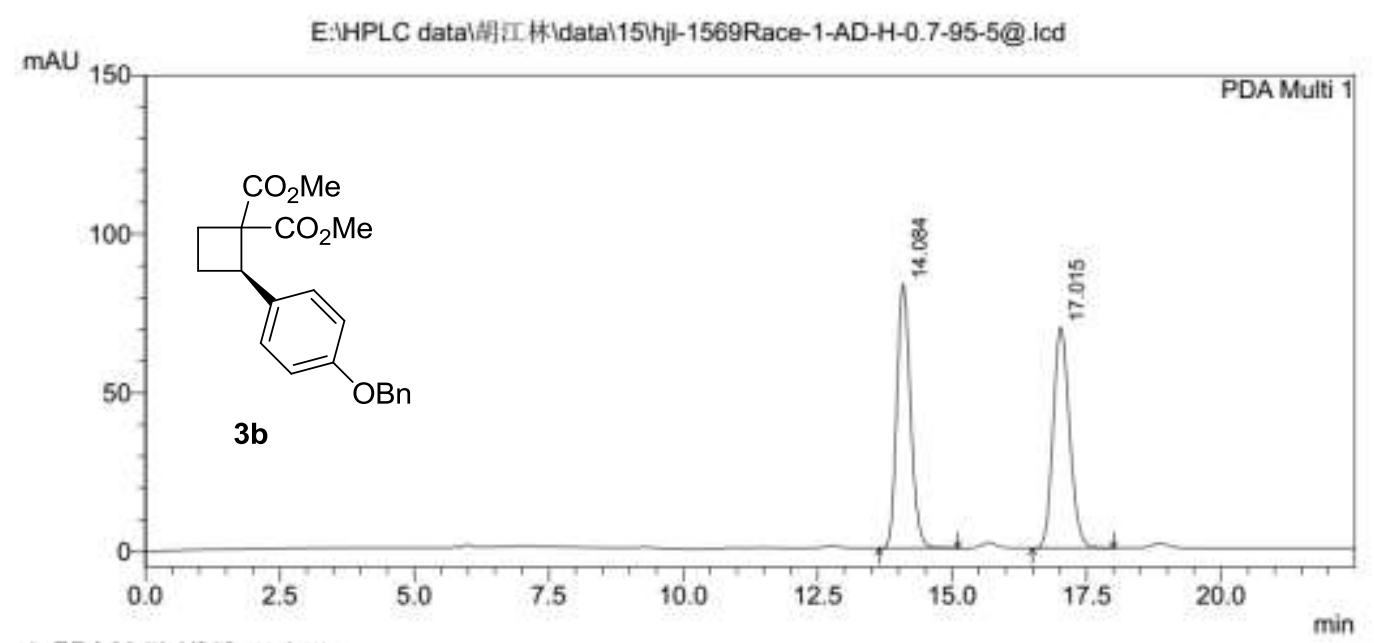

1 PDA Mult $1 / 240 \mathrm{~nm}$ 4nm

<Results>

PDA Ch1 $240 \mathrm{~nm} 4 \mathrm{~nm}$
\begin{tabular}{|l|r|r|r|r|r|r|}
\hline Peak \# & Ret. Time & \multicolumn{1}{|c|}{ Area } & Height & Area $\%$ & \multicolumn{1}{|c|}{ Height \% } & Mark \\
\hline 1 & 14.084 & 1471415 & 83199 & 49.902 & 54.437 & \\
\hline 2 & 17.015 & 1477174 & 69636 & 50.098 & 45.563 & \\
\hline Total & & 2948589 & 152835 & 100.000 & 100.000 & \\
\hline
\end{tabular}

\section{<Chromatogram>}

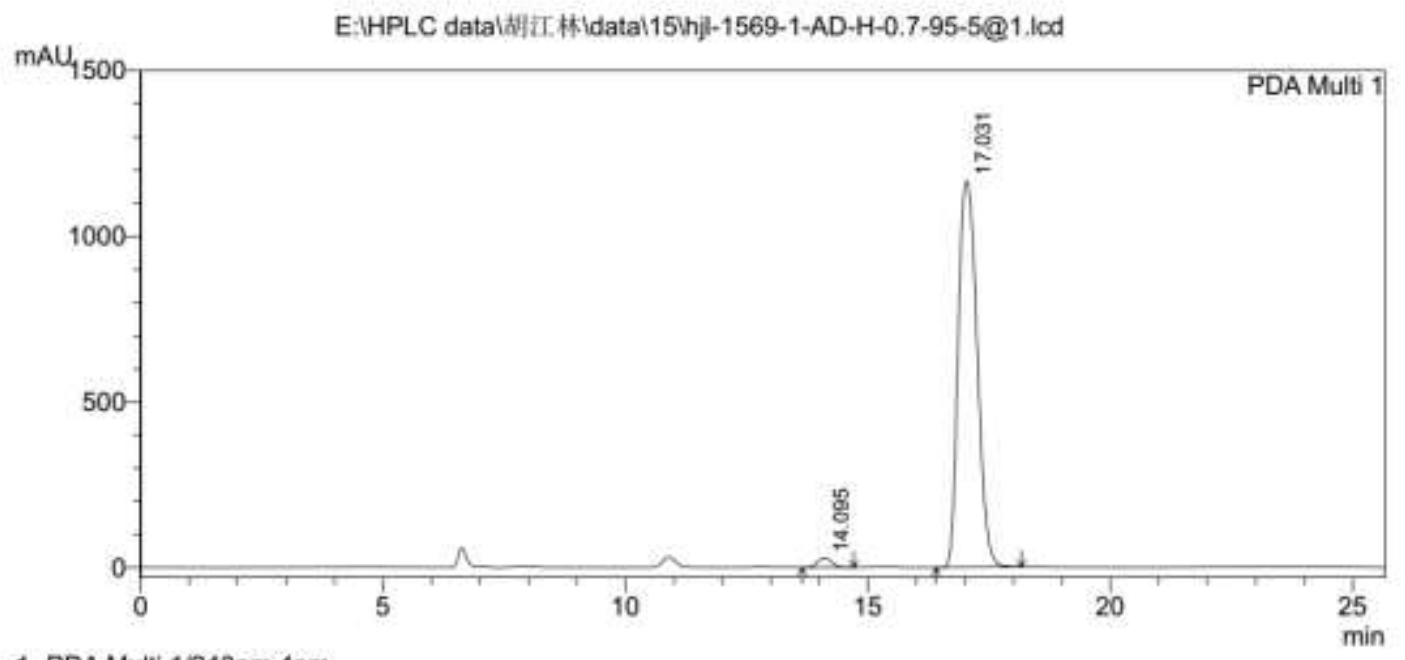

1 PDA Multi $1 / 240 \mathrm{~nm} 4 \mathrm{~nm}$

\section{$<$ Results>}

PDA Ch1 $240 \mathrm{~nm} 4 \mathrm{~nm}$
\begin{tabular}{|l|r|r|r|r|r|r|}
\hline Peak \# & Ret. Time & \multicolumn{1}{|c|}{ Area } & \multicolumn{1}{|c|}{ Height } & Area \% & Height $\%$ & Mark \\
\hline 1 & 14.095 & 591065 & 29272 & 1.841 & 2.445 & \\
\hline 2 & 17.031 & 31517728 & 1167758 & 98.159 & 97.555 & \\
\hline Total & & 32108793 & 1197030 & 100.000 & 100.000 & \\
\hline
\end{tabular}


$<$ Chromatogram>

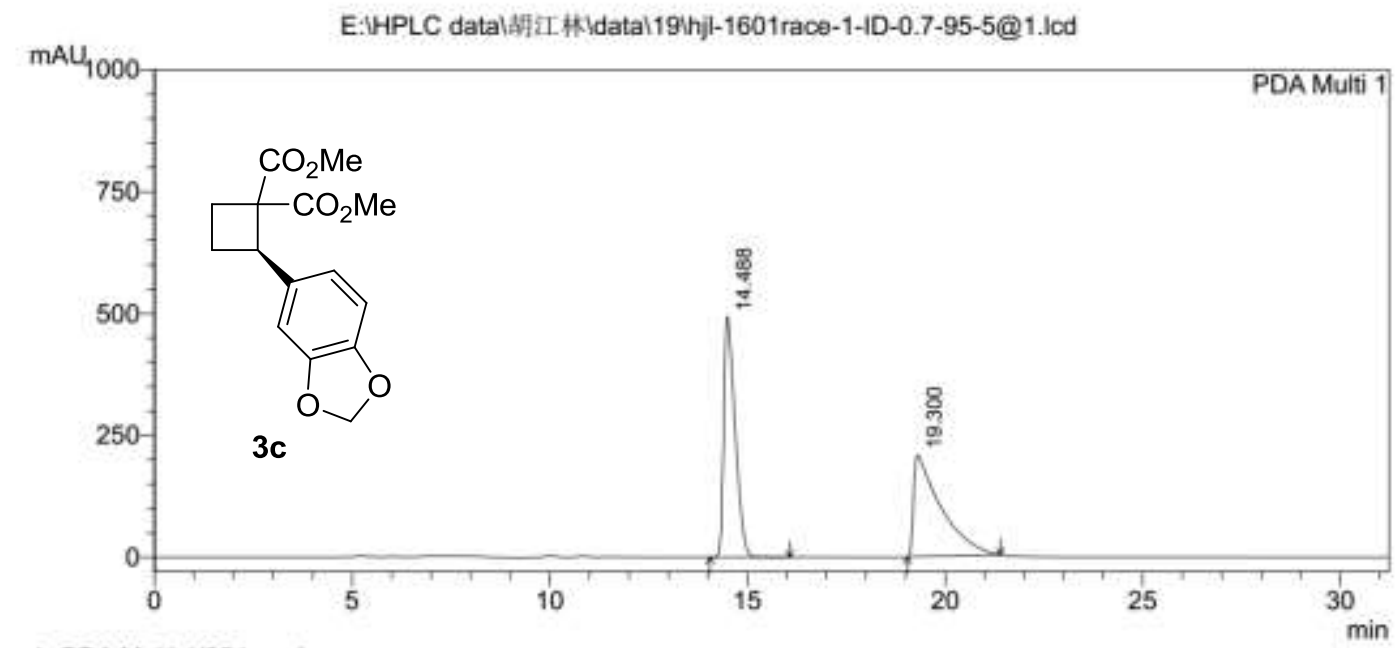

1 PDA Multi $1 / 254 \mathrm{~nm} 4 \mathrm{~nm}$

\section{$<$ Results>}

PDA Ch1 $254 \mathrm{~nm} 4 \mathrm{~nm}$
\begin{tabular}{|l|r|r|r|r|r|r|}
\hline Peak \# & Ret. Time & \multicolumn{1}{|c|}{ Area } & Height & Area $\%$ & Height $\%$ & Mark \\
\hline 1 & 14.488 & 9856509 & 494418 & 49.862 & 70.351 & $\mathrm{~S}$ \\
\hline 2 & 19.300 & 9911050 & 208370 & 50.138 & 29.649 & \\
\hline Total & & 19767559 & 702789 & 100.000 & 100.000 & \\
\hline
\end{tabular}

<Chromatogram>

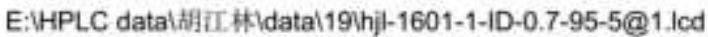

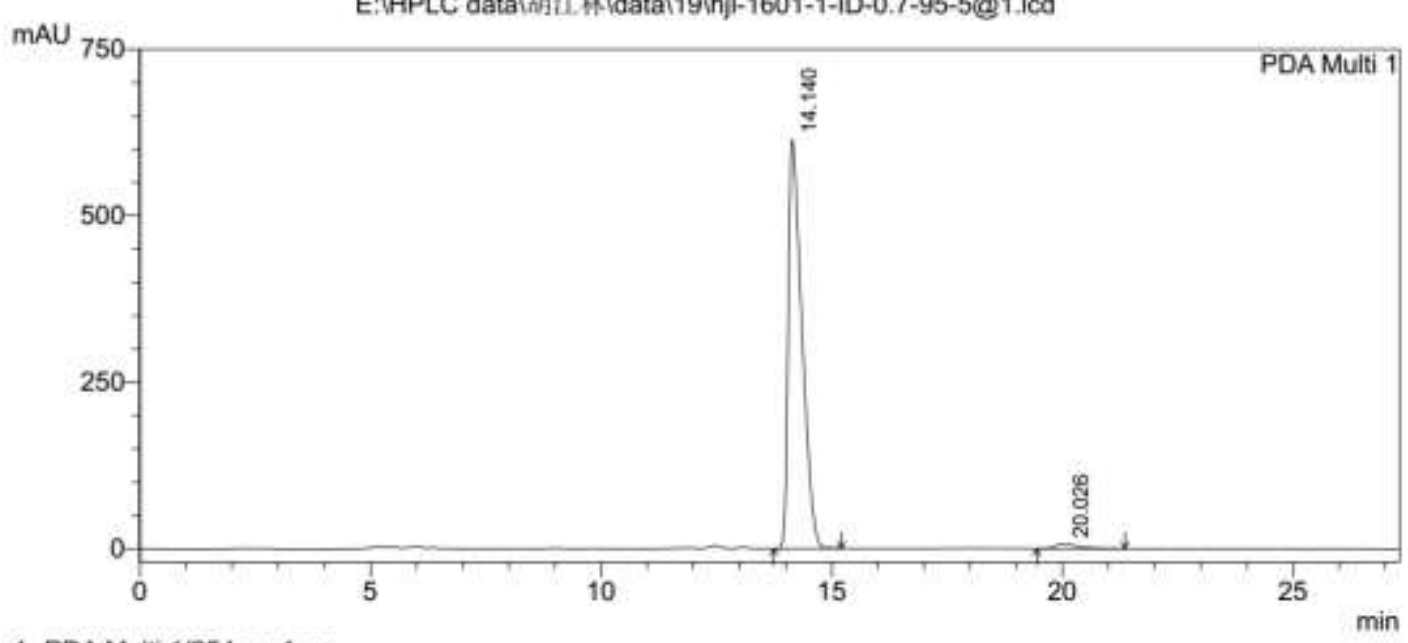

1 PDA Multi $1 / 254 \mathrm{~nm} 4 \mathrm{~nm}$

<Results>

PDA Ch1 254nm 4nm
\begin{tabular}{|l|r|r|r|r|r|r|r|}
\hline Peak \# & Ret. Time & \multicolumn{1}{|c|}{ Area } & \multicolumn{1}{|c|}{ Height } & Area \% & Height \% & Mark \\
\hline 1 & 14.140 & 12595328 & 614800 & 98.032 & 98.909 & \\
\hline 2 & 20.026 & 252819 & 6780 & 1.968 & 1.091 & \\
\hline Total & & 12848147 & 621580 & 100.000 & 100.000 \\
\hline
\end{tabular}


$<$ Chromatogram $>$

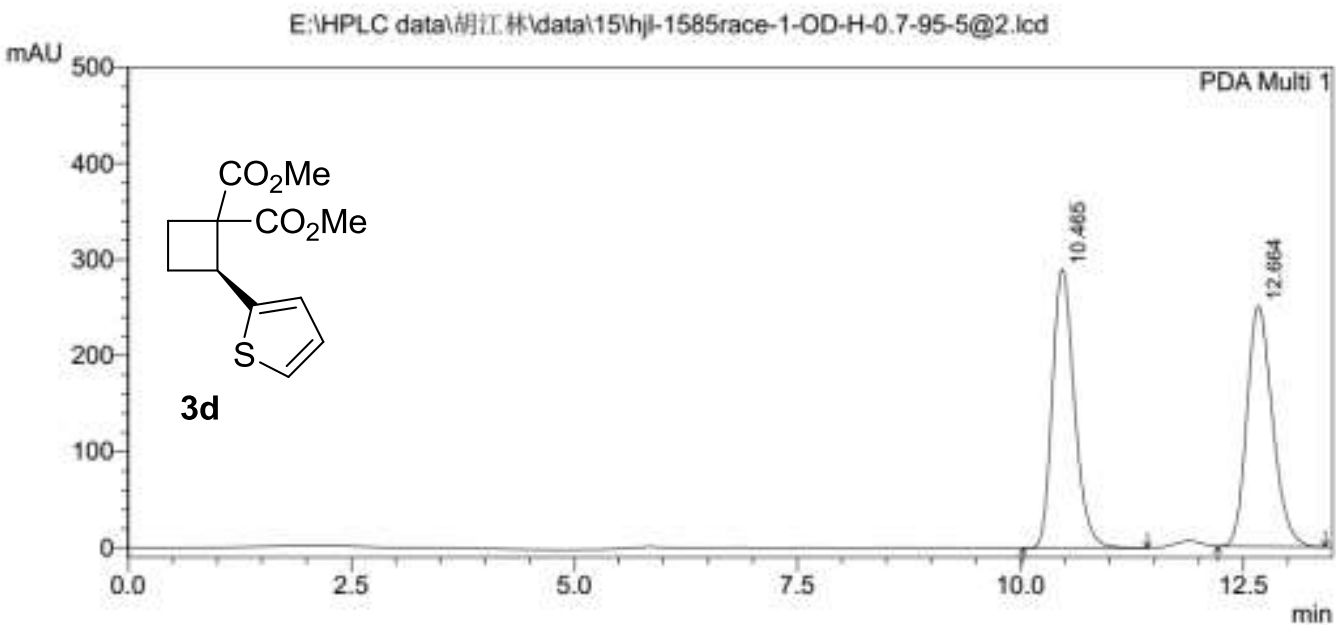

1 PDA Multi $1 / 250 \mathrm{~nm} 4 \mathrm{~nm}$

<Results>

PDA Ch1 $250 \mathrm{~nm} 4 \mathrm{~nm}$
\begin{tabular}{|l|r|r|r|r|r|r|}
\hline Peak \# & Ret. Time & \multicolumn{1}{|c|}{ Area } & Height & Area $\%$ & Height $\%$ & Mark \\
\hline 1 & 10.465 & 4986187 & 290250 & 49.848 & 53.664 & \\
\hline 2 & 12.664 & 5016611 & 250611 & 50.152 & 46.336 & \\
\hline Total & & 10002798 & 540861 & 100.000 & 100.000 \\
\hline
\end{tabular}

$<$ Chromatogram>

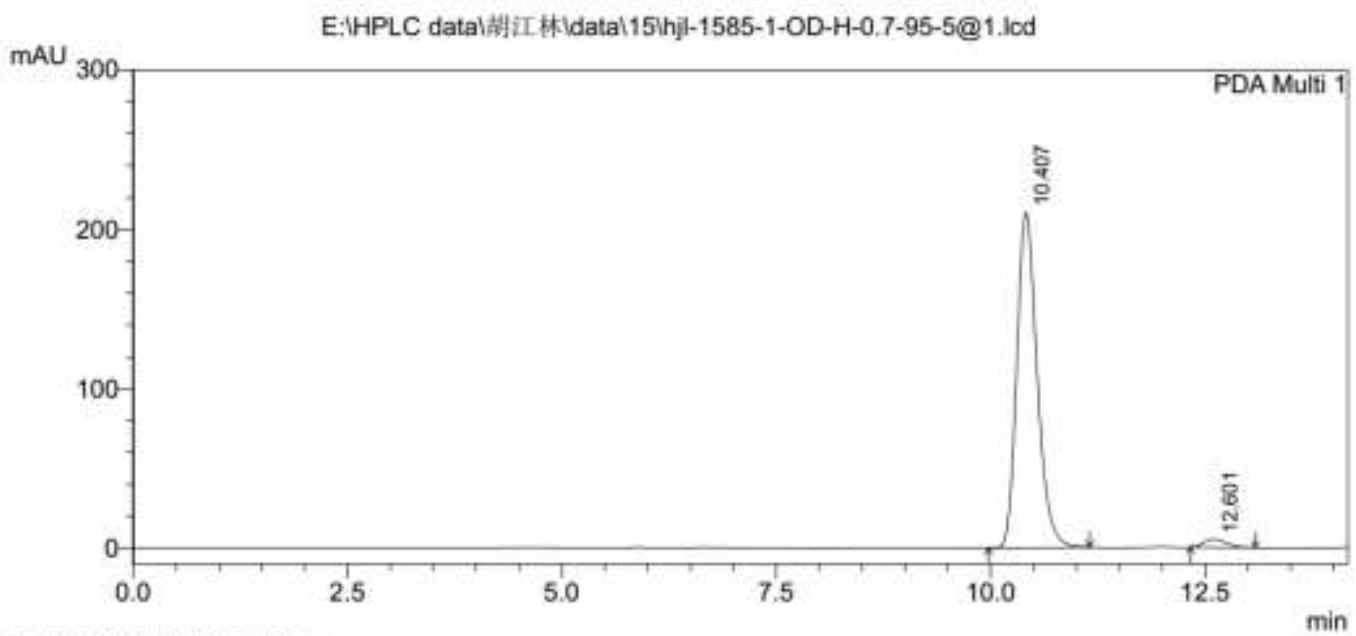

1 PDA Multi $1 / 250 \mathrm{~nm} 4 \mathrm{~nm}$

\section{$<$ Results>}

PDACh $1250 \mathrm{~nm} 4 \mathrm{~nm}$
\begin{tabular}{|l|r|r|r|r|r|r|}
\hline Peak \# & Ret. Time & \multicolumn{1}{|c|}{ Area } & Height & \multicolumn{1}{|c|}{ Area $\%$} & Height $\%$ & Mark \\
\hline 1 & 10.407 & 3467359 & 210627 & 97.449 & 97.681 & \\
\hline 2 & 12.601 & 90772 & 5000 & 2.551 & 2.319 & \\
\hline Total & & 3558131 & 215627 & 100.000 & 100.000 & \\
\hline
\end{tabular}


$<$ Chromatogram>

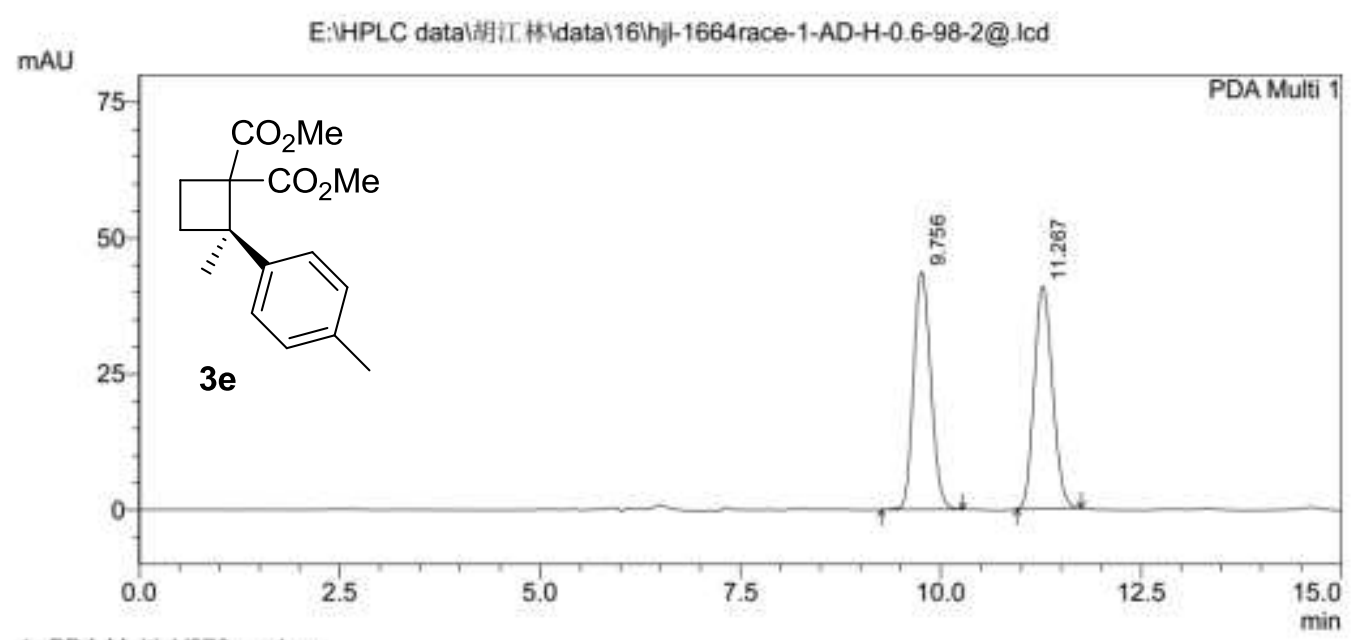

1 PDA Multi $1 / 270 \mathrm{~nm} 4 \mathrm{~nm}$

\section{<Results>}

PDA Ch1 $270 \mathrm{~nm}$ 4nm
\begin{tabular}{|l|r|r|r|r|r|r|}
\hline Peak \# & Ret Time & \multicolumn{1}{|c|}{ Area } & Height & Area $\%$ & Height $\%$ & Mark \\
\hline 1 & 9.756 & 650993 & 43777 & 50.114 & 51.635 & \\
\hline 2 & 11.267 & 648037 & 41004 & 49.886 & 48.365 & \\
\hline Total & & 1299030 & 84782 & 100.000 & 100.000 & \\
\hline
\end{tabular}

\section{$<$ Chromatogram $>$}

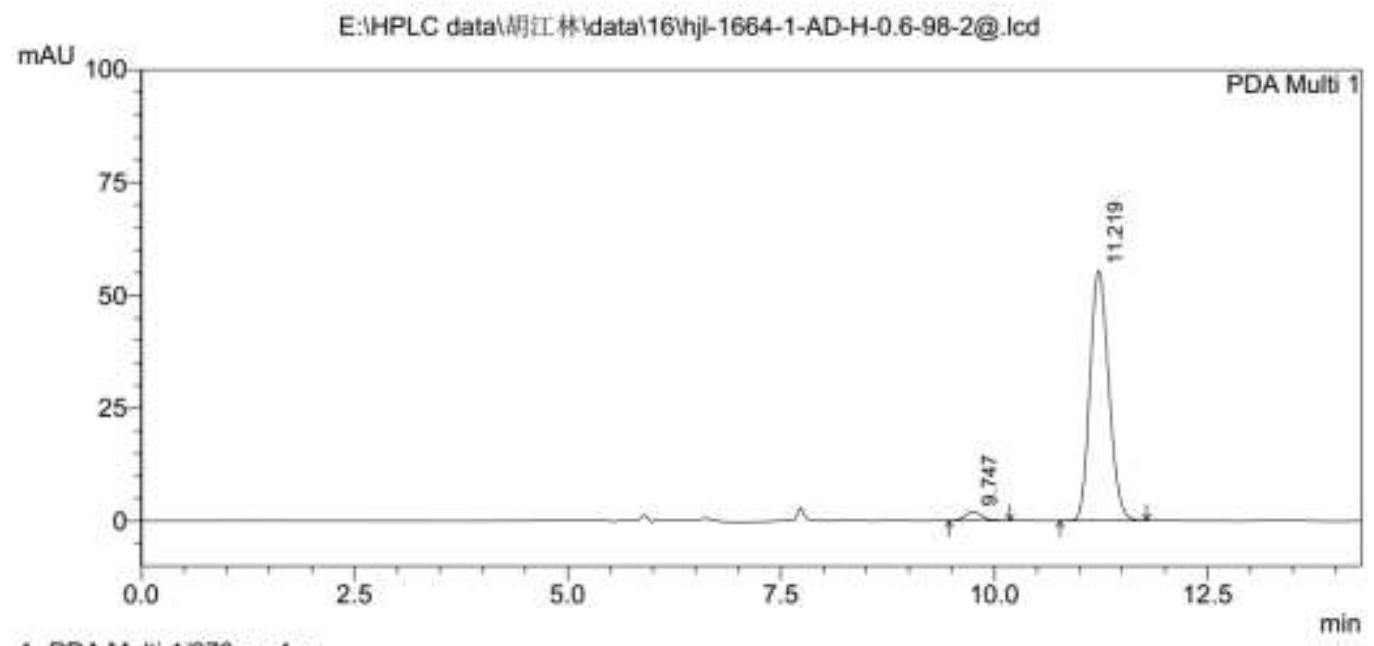

1 PDA Multi $1 / 270 \mathrm{~nm} 4 \mathrm{~nm}$

\section{<Results>}

PDA Ch1 270nm 4nm
\begin{tabular}{|l|r|r|r|r|r|r|}
\hline Peak \# & Ret. Time & \multicolumn{1}{|c|}{ Area } & Height & Area \% & Height \% & Mark \\
\hline 1 & 9.747 & 26483 & 1907 & 3.025 & 3.322 & \\
\hline 2 & 11.219 & 848901 & 55490 & 96.975 & 96.678 & \\
\hline Total & & 875383 & 57397 & 100.000 & 100.000 & \\
\hline
\end{tabular}




\section{$<$ Chromatogram $>$}

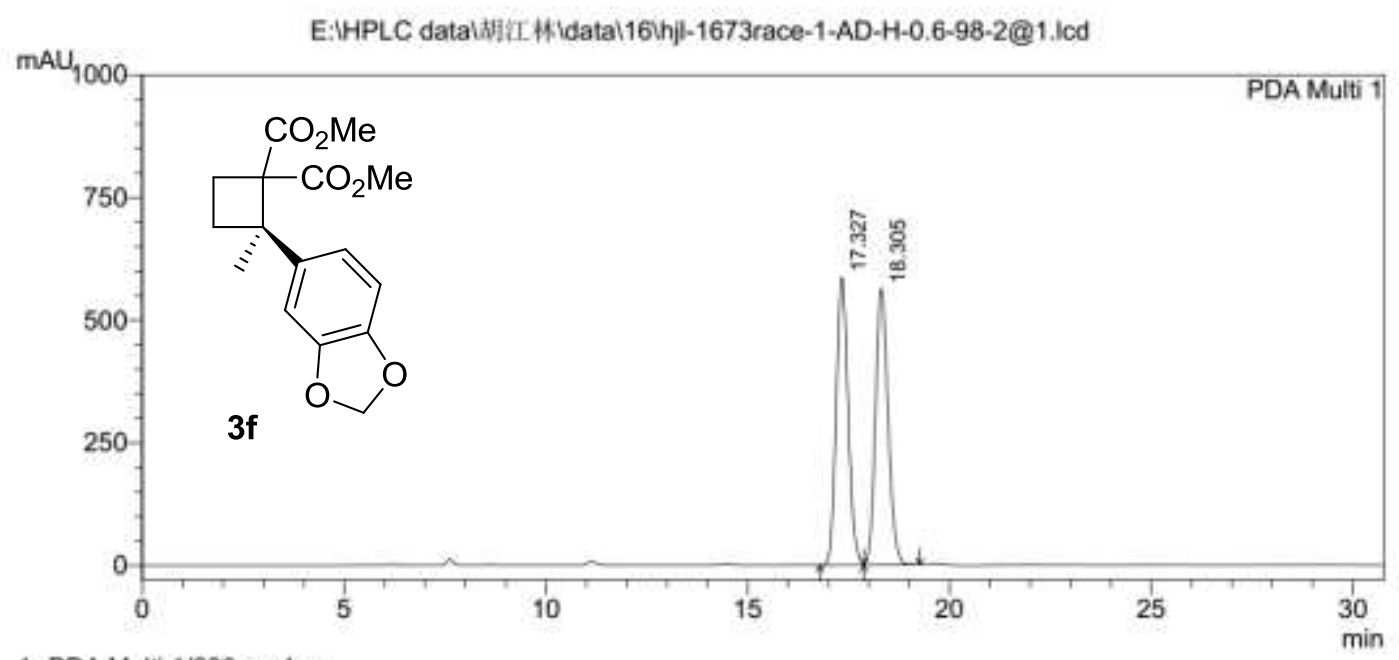

1 PDA Multi 1/230nm 4nm

\section{<Results>}

PDA Ch1 $230 \mathrm{~nm}$ 4nm
\begin{tabular}{|l|r|r|r|r|r|r|}
\hline Peak \# & Ret. Time & Area & \multicolumn{1}{|c|}{ Height } & Area $\%$ & Height \% & Mark \\
\hline 1 & 17.327 & 12048776 & 587154 & 49.670 & 51.033 & \\
\hline 2 & 18.305 & 12208850 & 563379 & 50.330 & 48.967 & V \\
\hline Total & & 24257626 & 1150534 & 100.000 & 100.000 & \\
\hline
\end{tabular}

<Chromatogram>

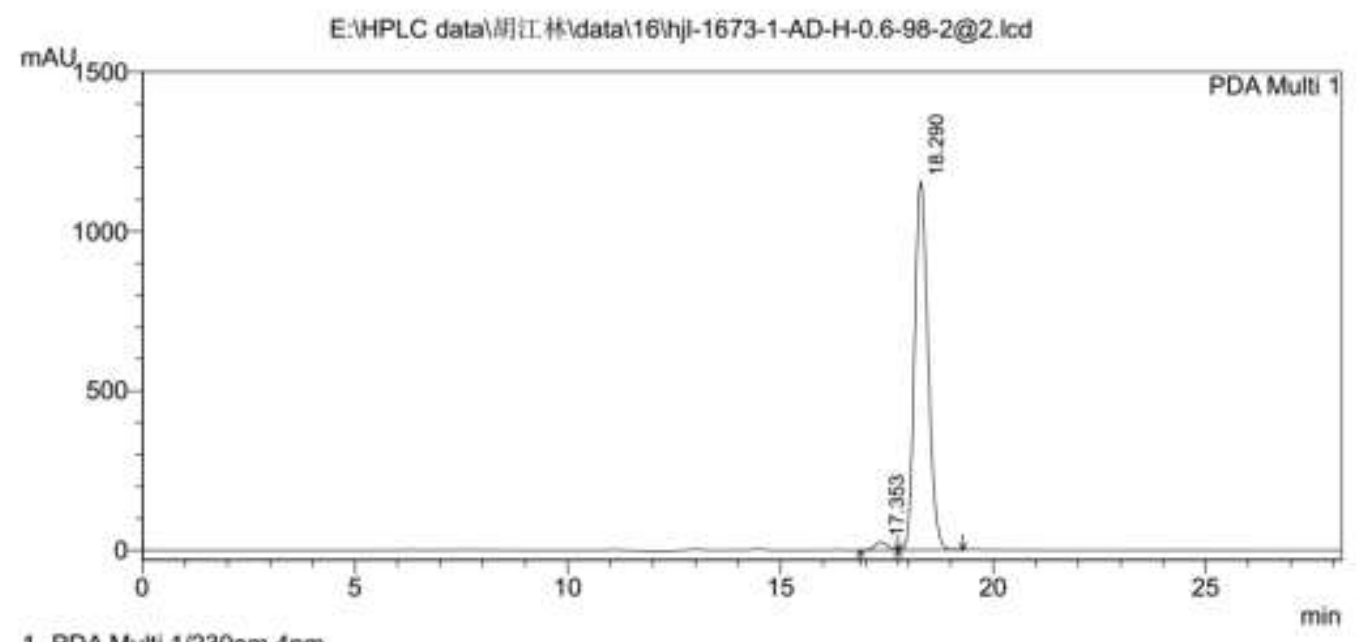

1 PDA Multi $1 / 230 \mathrm{~nm} 4 \mathrm{~nm}$

<Results>

PDACh1 $230 \mathrm{~nm} 4 \mathrm{~nm}$
\begin{tabular}{|l|r|r|r|r|r|r|}
\hline Peak \# & Ret. Time & \multicolumn{1}{c|}{ Area } & Height & Area $\%$ & Height $\%$ & Mark \\
\hline 1 & 17.353 & 530811 & 25708 & 2.047 & 2.176 & \\
\hline 2 & 18.290 & 25398202 & 1155890 & 97.953 & 97.824 & V \\
\hline Total & & 25929014 & 1181598 & 100.000 & 100.000 & \\
\hline
\end{tabular}


$<$ Chromatogram>

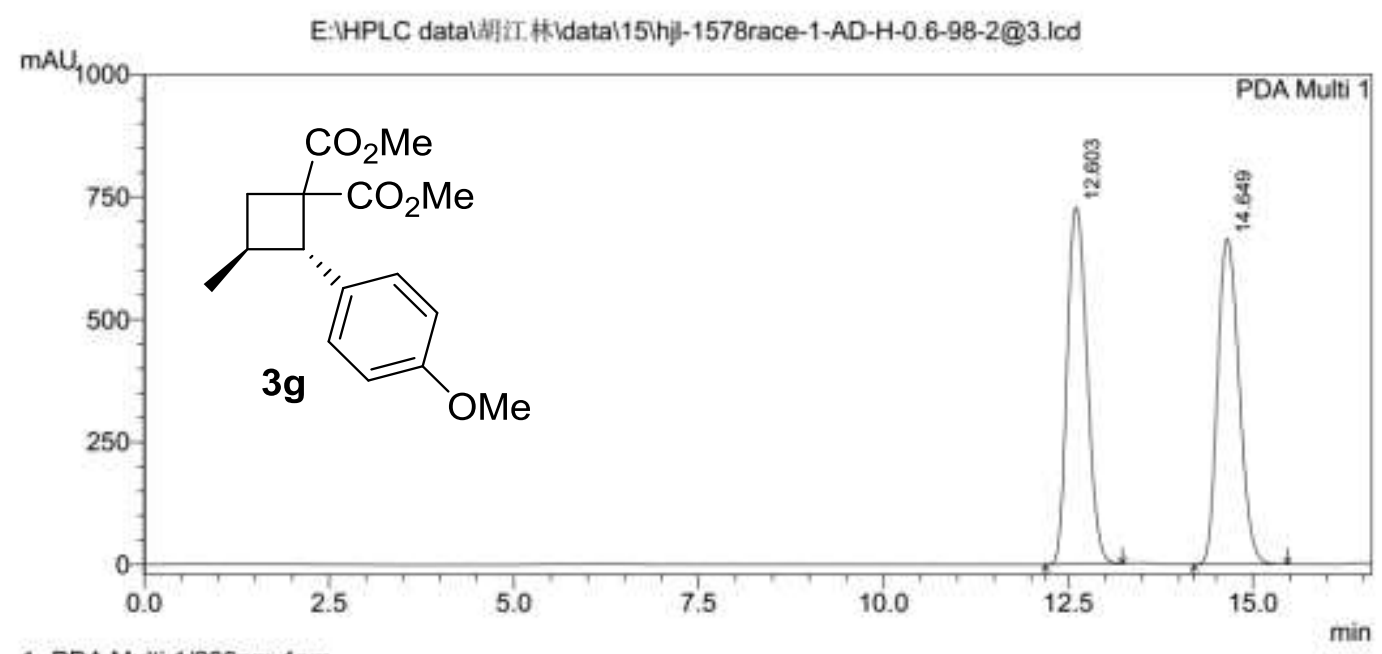

1 PDA Multi $1 / 280 \mathrm{~nm} 4 \mathrm{~nm}$

\section{<Results>}

PDA Ch1 2 BOnm $4 \mathrm{~nm}$
\begin{tabular}{|l|r|r|r|r|r|r|}
\hline Peak \# & Ret. Time & \multicolumn{1}{c|}{ Area } & Height & Area $\%$ & Height $\%$ & Mark \\
\hline 1 & 12.603 & 13584682 & 729047 & 50.211 & 52.294 & \\
\hline 2 & 14.649 & 13470624 & 665093 & 49.789 & 47.706 & \\
\hline Total & & 27055305 & 1394140 & 100.000 & 100.000 & \\
\hline
\end{tabular}

\section{$<$ Chromatogram>}

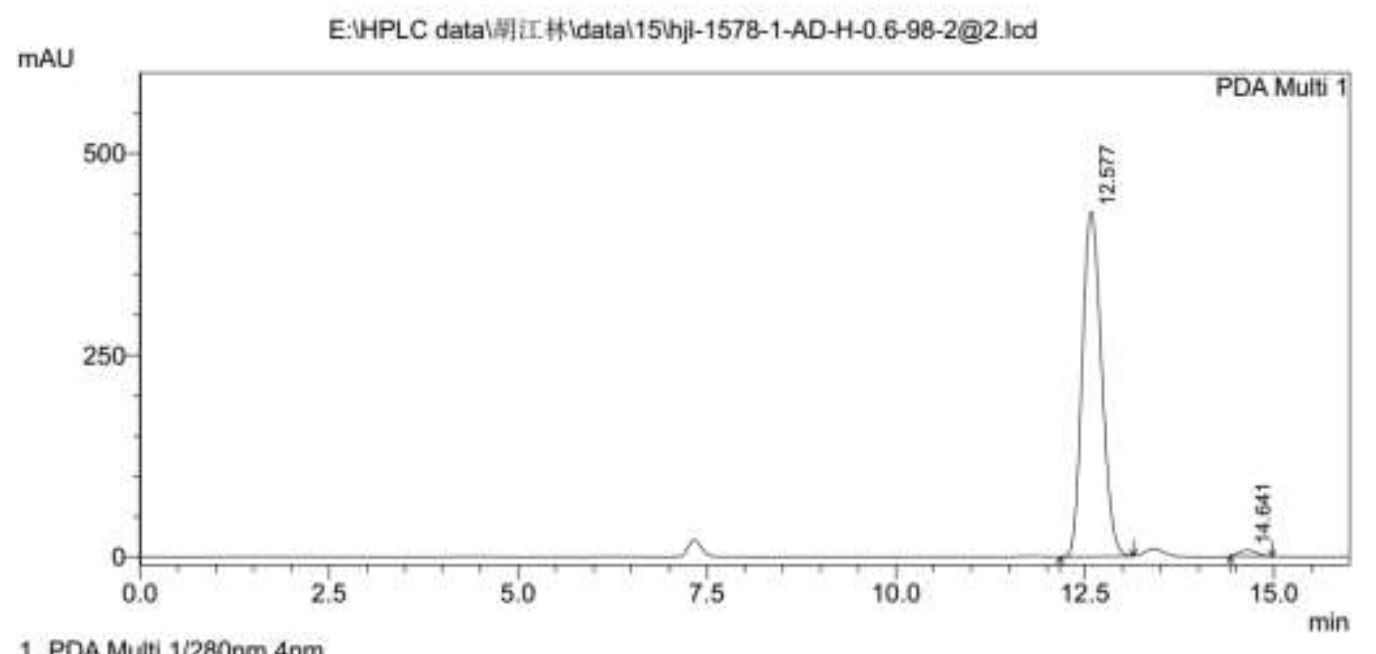

1 PDA Multi $1 / 280 \mathrm{~nm} 4 \mathrm{~nm}$

\section{<Results>}

PDA Ch1 $280 \mathrm{~nm} 4 \mathrm{~nm}$
\begin{tabular}{|l|r|r|r|r|r|r|}
\hline Peak \# & Ret. Time & \multicolumn{1}{c|}{ Area } & Height & Area $\%$ & Height $\%$ & Mark \\
\hline 1 & 12.577 & 7583020 & 427610 & 98.434 & 98.309 & \\
\hline 2 & 14.641 & 120648 & 7357 & 1.566 & 1.691 & \\
\hline Total & & 7703669 & 434967 & 100.000 & 100.000 & \\
\hline
\end{tabular}


$<$ Chromatogram>

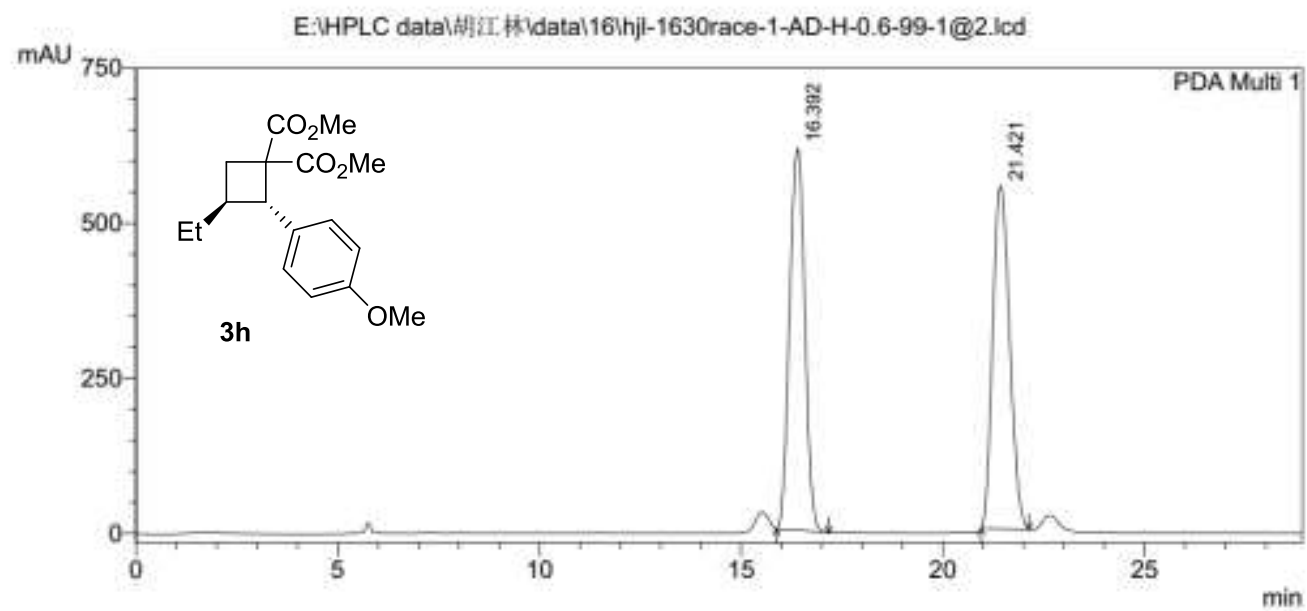

1 PDA Multi $1 / 280 \mathrm{~nm} 4 \mathrm{~nm}$

\section{$<$ Results>}

PDA Ch $1280 \mathrm{~nm} 4 \mathrm{~nm}$
\begin{tabular}{|l|r|r|r|r|r|r|}
\hline Peak. & Ret. Time & \multicolumn{1}{c|}{ Area } & \multicolumn{1}{c|}{ Height } & Area $\%$ & Height $\%$ & Mark \\
\hline 1 & 16.392 & 16126918 & 616245 & 50.036 & 52.677 & \\
\hline 2 & 21.421 & 16103959 & 553611 & 49.964 & 47.323 & \\
\hline Total & & 32230877 & 1169856 & 100.000 & 100.000 & \\
\hline
\end{tabular}

\section{$<$ Chromatogram $>$}

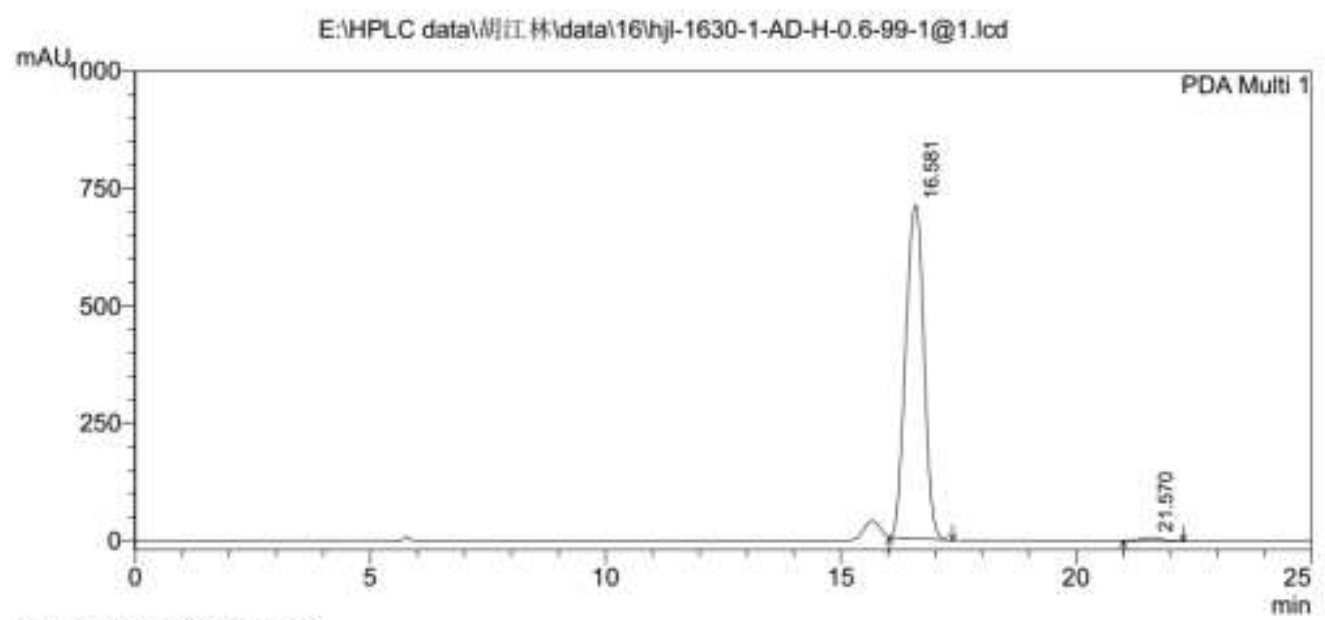

1 PDA Multi $1 / 280 \mathrm{~nm} 4 \mathrm{~nm}$

\section{<Results>}

PDA Ch1 $280 \mathrm{~nm} 4 \mathrm{~nm}$
\begin{tabular}{|l|r|r|r|r|r|r|}
\hline Peak स & Ret. Time & \multicolumn{1}{|c|}{ Area } & \multicolumn{1}{c|}{ Height } & Area \% & Height \% & Mark \\
\hline 1 & 16.581 & 19083002 & 711130 & 98.899 & 98.907 & \\
\hline 2 & 21.570 & 212400 & 7861 & 1.101 & 1.093 & \\
\hline Total & & 19295402 & 718991 & 100.000 & 100.000 & \\
\hline
\end{tabular}


$<$ Chromatogram $>$

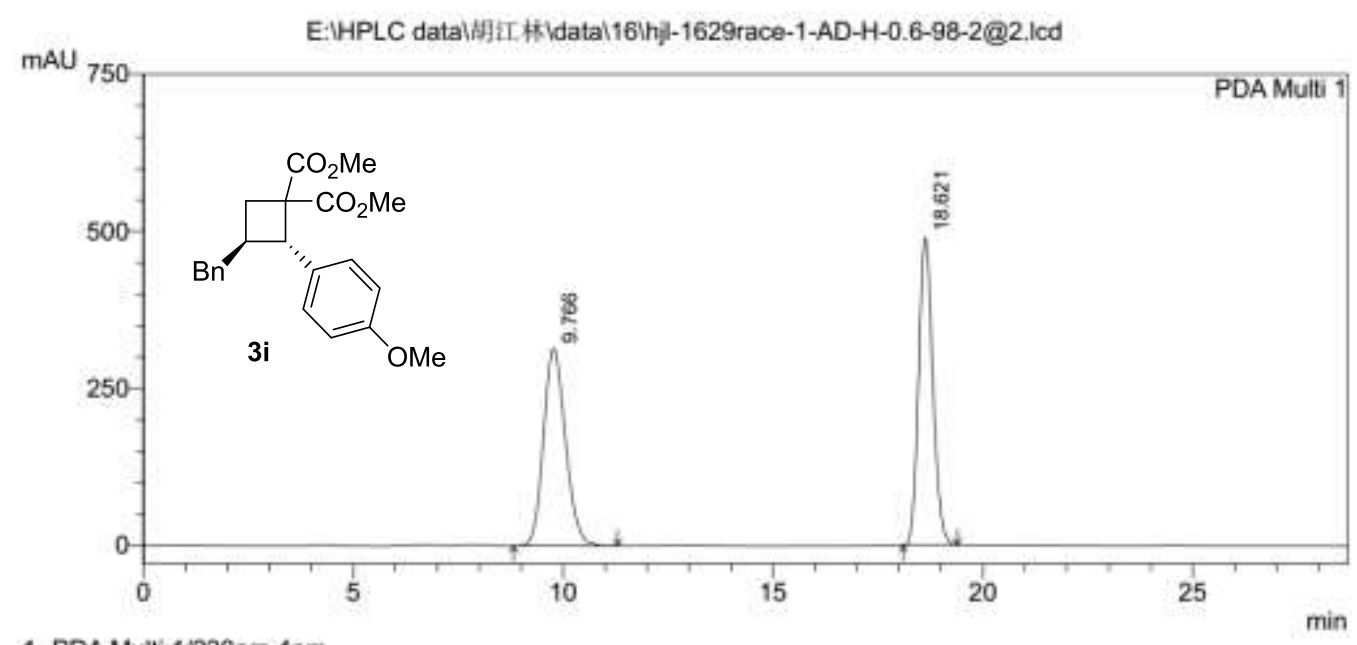

1 PDA Multi $1 / 230 \mathrm{~nm} 4 \mathrm{~nm}$

<Results>

PDA Ch1 230nm 4nm
\begin{tabular}{|l|r|r|r|r|r|r|}
\hline Peak \# & Ret. Time & \multicolumn{1}{|c|}{ Area } & Height & Area \% & Height \% & Mark \\
\hline 1 & 9.766 & 11403973 & 313658 & 49.655 & 39.039 \\
\hline 2 & 18.621 & 11562430 & 489796 & 50.345 & 60.961 \\
\hline Total & & 22966404 & 803454 & 100.000 & 100.000 \\
\hline
\end{tabular}

\section{$<$ Chromatogram $>$}

E:IHPLC datal调红. Hoidatal16hil-1629b-1-AD-H-0.6-98-2@1.led

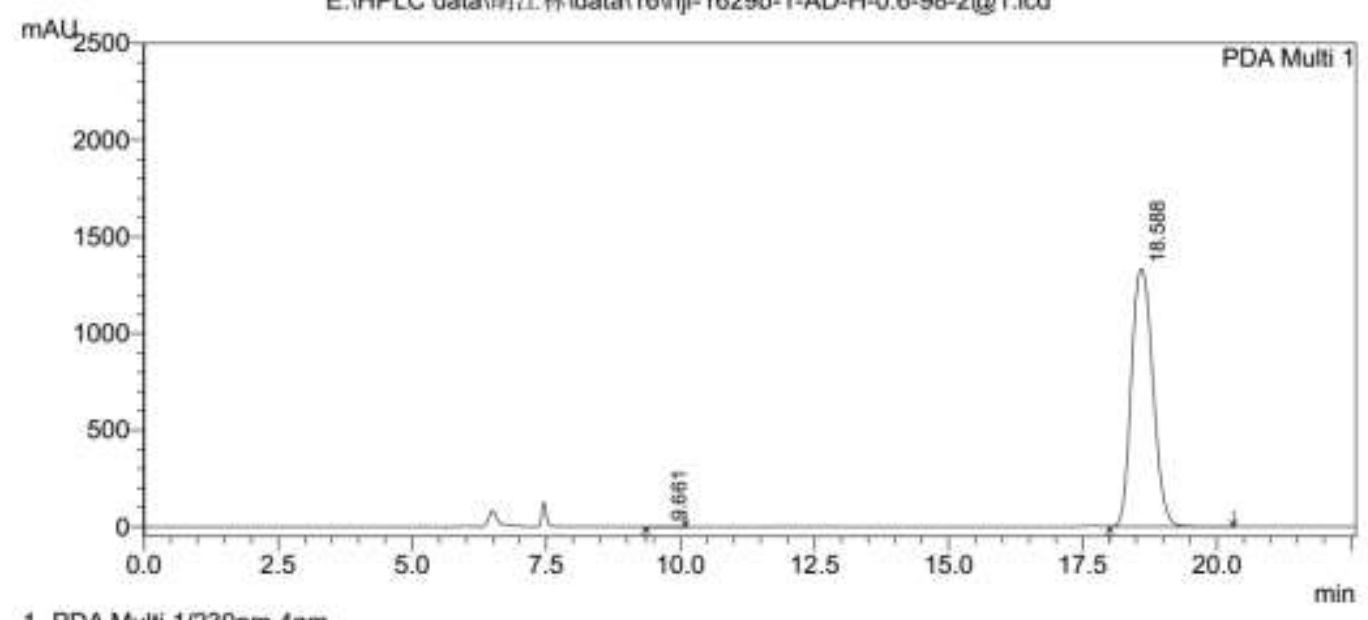

1 PDA Multi $1 / 230 \mathrm{~nm} 4 \mathrm{~nm}$

$<$ Results>

PDA Ch1 230nm 4nm

\begin{tabular}{|l|r|r|r|r|r|r|}
\hline Peak\# & Ret. Time & \multicolumn{1}{|c|}{ Area } & Height & Area \% & Height \% & Mark \\
\hline 1 & 9.661 & 7319 & 468 & 0.020 & 0.035 & \\
\hline 2 & 18.588 & 37050362 & 1333773 & 99.980 & 99.965 & \\
\hline Total & & 37057681 & 1334241 & 100.000 & 100.000 & \\
\hline
\end{tabular}


$<$ Chromatogram $>$

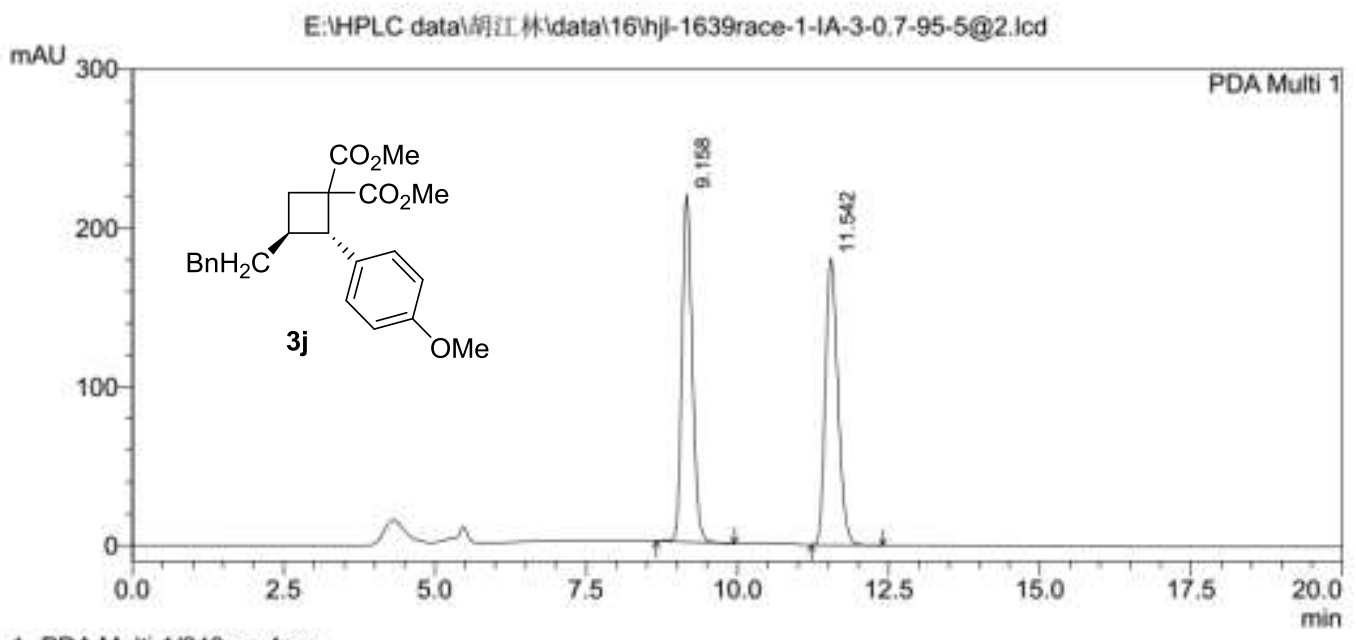

1 PDA Multi $1 / 240 \mathrm{~nm} 4 \mathrm{~nm}$

\section{<Results>}

PDA Ch1 $240 \mathrm{~nm} 4 \mathrm{~nm}$
\begin{tabular}{|l|r|r|r|r|r|r|}
\hline Peak \# & Ret. Time & \multicolumn{1}{|c|}{ Area } & Height & Area $\%$ & Height $\%$ & Mark \\
\hline 1 & 9.158 & 2598189 & 219047 & 50.041 & 54.813 & \\
\hline 2 & 11.542 & 2593917 & 180580 & 49.959 & 45.187 & \\
\hline Total & & 5192106 & 399627 & 100.000 & 100.000 & \\
\hline
\end{tabular}

\section{$<$ Chromatogram $>$}

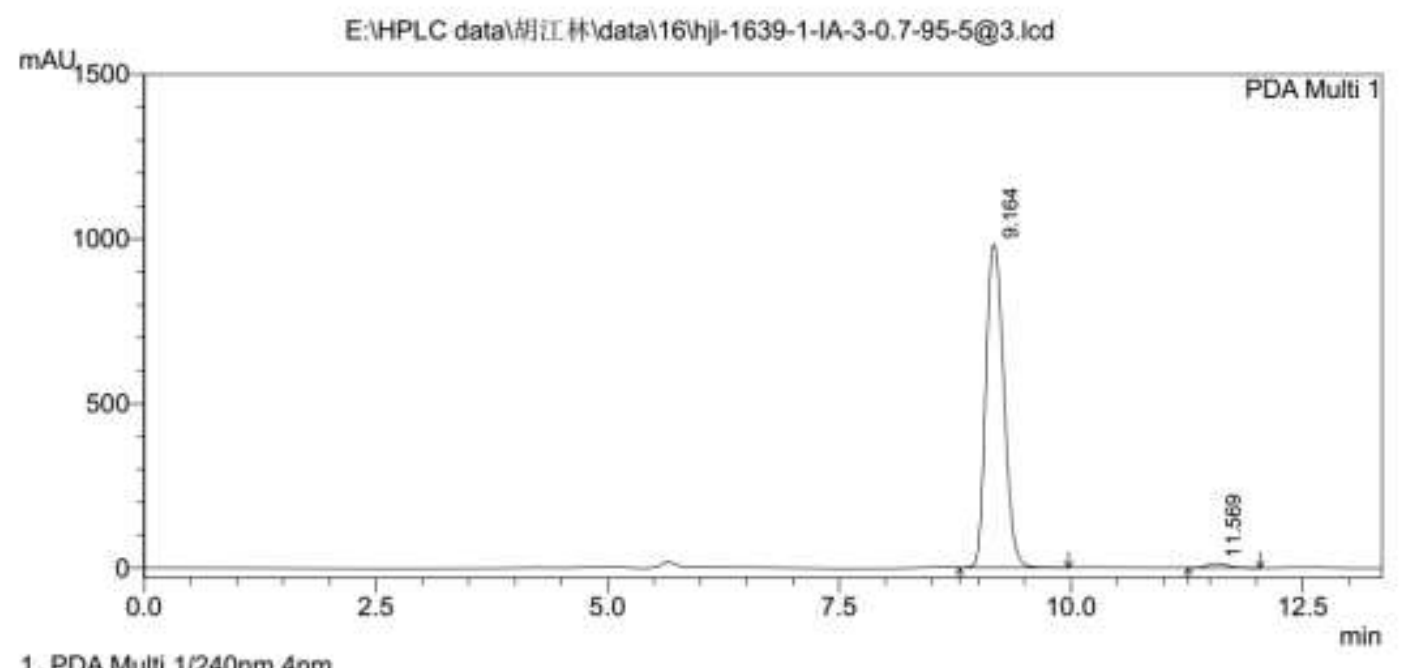

1. PDA Multi $1 / 240 \mathrm{~nm} 4 \mathrm{~nm}$

<Results>

PDA Ch1 240nm 4nm
\begin{tabular}{|l|r|r|r|r|r|r|}
\hline Peak \# & Ret. Time & \multicolumn{1}{|c|}{ Area } & \multicolumn{1}{|c|}{ Height } & Area \% & Height \% & Mark \\
\hline 1 & 9.164 & 13278501 & 982951 & 98.458 & 98.548 & \\
\hline 2 & 11.569 & 207938 & 14487 & 1.542 & 1.452 & \\
\hline Total & & 13486439 & 997438 & 100.000 & 100.000 & \\
\hline
\end{tabular}


$<$ Chromatogram $>$

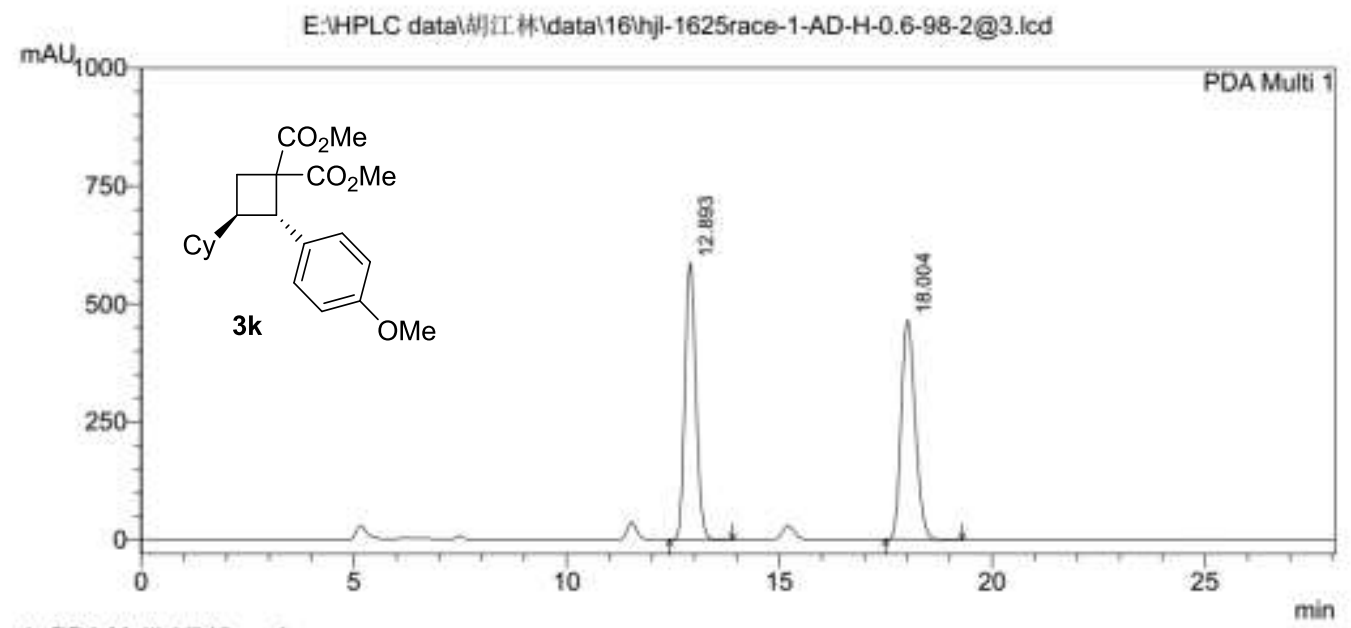

1 PDA Multi 1/240nm 4nm

<Results>

PDA Ch1 240nm 4nm

\begin{tabular}{|l|r|r|r|r|r|r|}
\hline Peak H & Ret Time & \multicolumn{1}{|c|}{ Area } & \multicolumn{1}{|c|}{ Height } & \multicolumn{1}{|c|}{ Area \% } & Height \% & Mark \\
\hline 1 & 12.893 & 10529995 & 586874 & 49.690 & 55.774 & \\
\hline 2 & 18.004 & 10661379 & 465370 & 50.310 & 44.226 & \\
\hline Total & & 21191373 & 1052244 & 100.000 & 100.000 & \\
\hline
\end{tabular}

\section{<Chromatogram>}

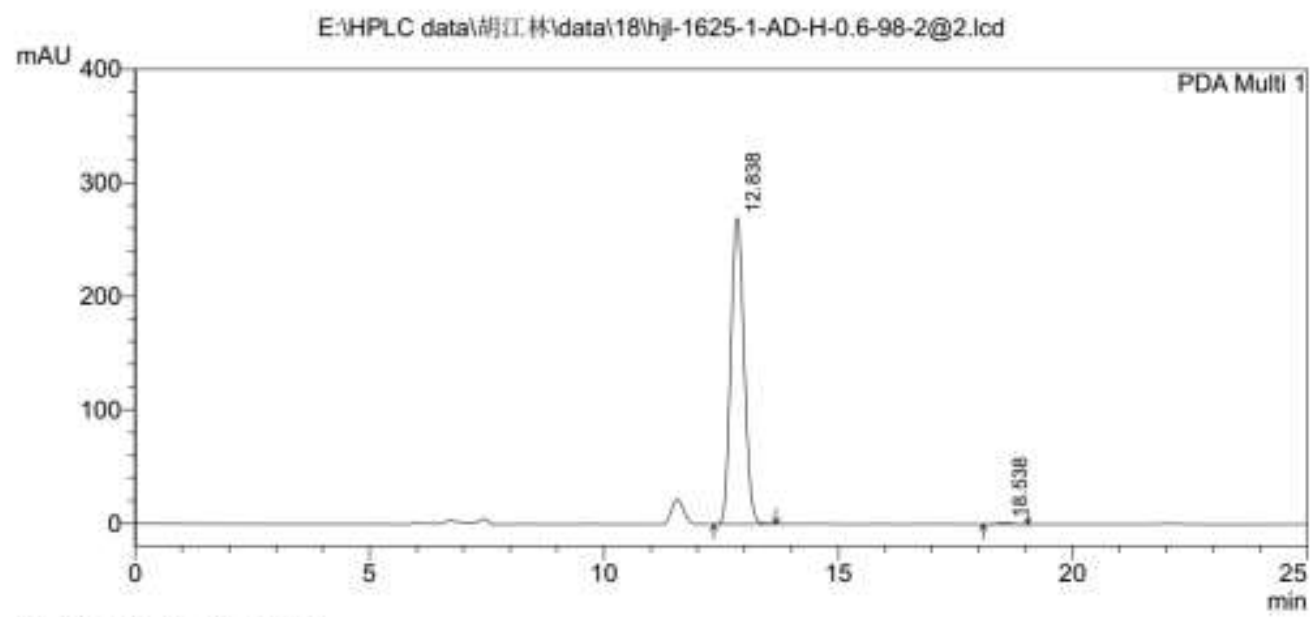

1. PDA Multi $1 / 240 \mathrm{~nm} 4 \mathrm{~nm}$

\section{<Results>}

PDA Ch1 $240 \mathrm{~nm} 4 \mathrm{~nm}$
\begin{tabular}{|l|r|r|r|r|r|r|}
\hline Peak \# & Ret. Time & \multicolumn{1}{|c|}{ Area } & \multicolumn{1}{c|}{ Height } & \multicolumn{1}{|c|}{ Area \% } & Height \% & Mark \\
\hline 1 & 12.838 & 5297428 & 269879 & 99.555 & 99.642 & \\
\hline 2 & 18.538 & 23692 & 969 & 0.445 & 0.358 \\
\hline Total & & 5321120 & 270848 & 100.000 & 100.000 \\
\hline
\end{tabular}


$<$ Chromatogram>

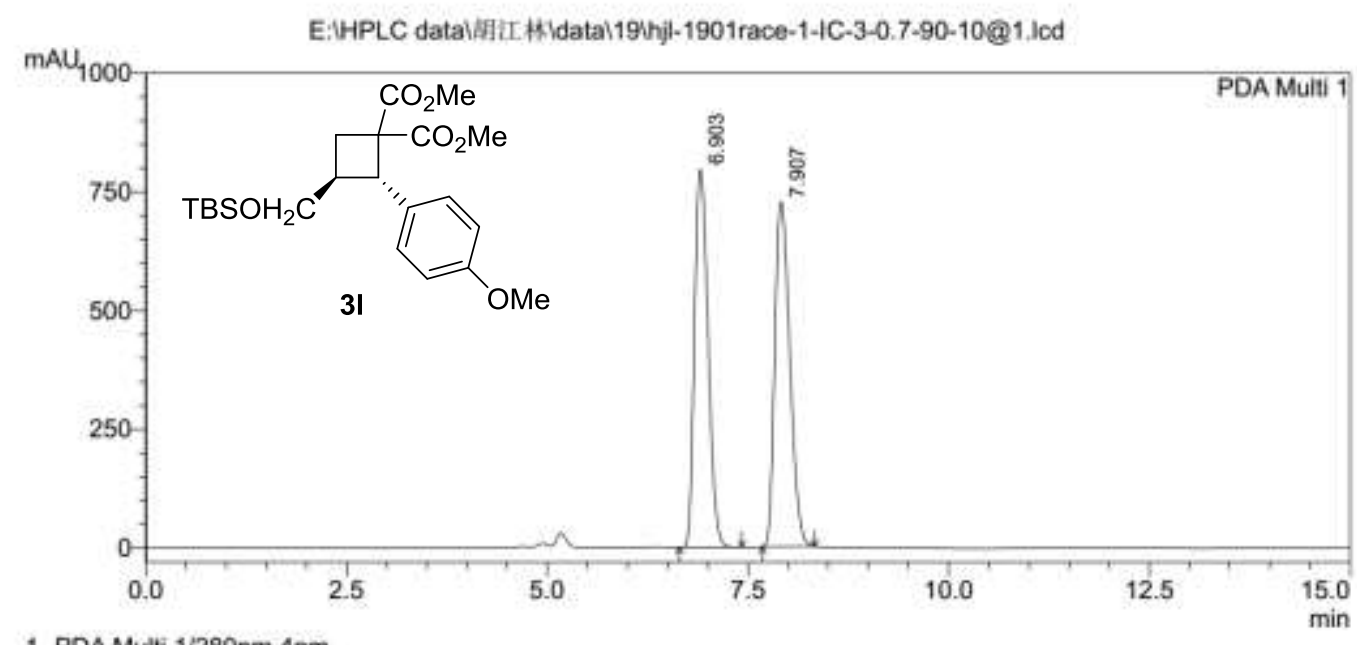

1 PDA Multi $1 / 280 \mathrm{~nm} 4 \mathrm{~nm}$

<Results>

PDA Ch1 $280 \mathrm{~nm} 4 \mathrm{~nm}$
\begin{tabular}{|l|r|r|r|r|r|r|}
\hline Peak \# & Ret. Time & \multicolumn{1}{c|}{ Area } & \multicolumn{1}{c|}{ Height } & Area $\%$ & Height $\%$ & Mark \\
\hline 1 & 6.903 & 9318301 & 796676 & 49.573 & 52.295 & \\
\hline 2 & 7.907 & 9478682 & 726742 & 50.427 & 47.705 & \\
\hline Total & & 18796983 & 1523418 & 100.000 & 100.000 & \\
\hline
\end{tabular}

\section{$<$ Chromatogram $>$}

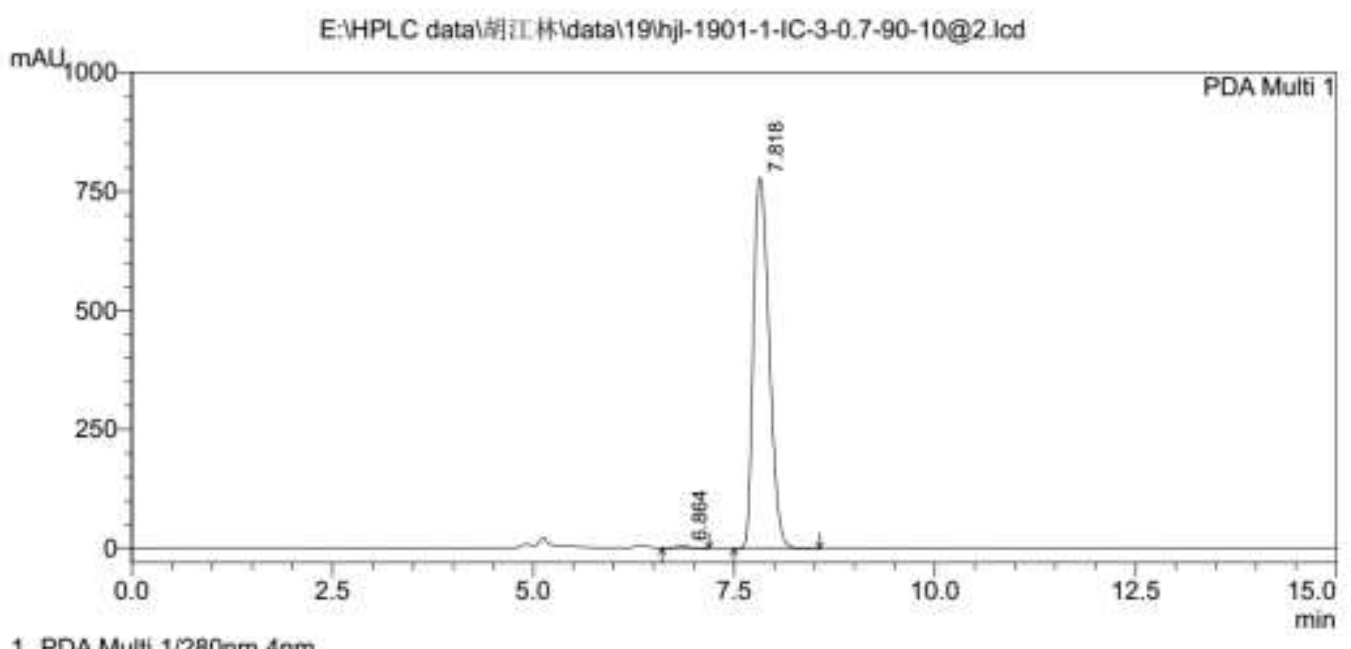

1 PDA Multi $1 / 280 \mathrm{~nm} 4 \mathrm{~nm}$

\section{<Results>}

PDA Ch1 280nm 4nm
\begin{tabular}{|l|r|r|r|r|r|r|}
\hline Peak \# & Ret. Time & \multicolumn{1}{|c|}{ Area } & Height & Area $\%$ & Height $\%$ & Mark \\
\hline 1 & 6.864 & 49551 & 4630 & 0.463 & 0.591 & \\
\hline 2 & 7.818 & 10644303 & 779276 & 99.537 & 99.409 & \\
\hline Total & & 10693854 & 783906 & 100.000 & 100.000 & \\
\hline
\end{tabular}


$<$ Chromatogram>

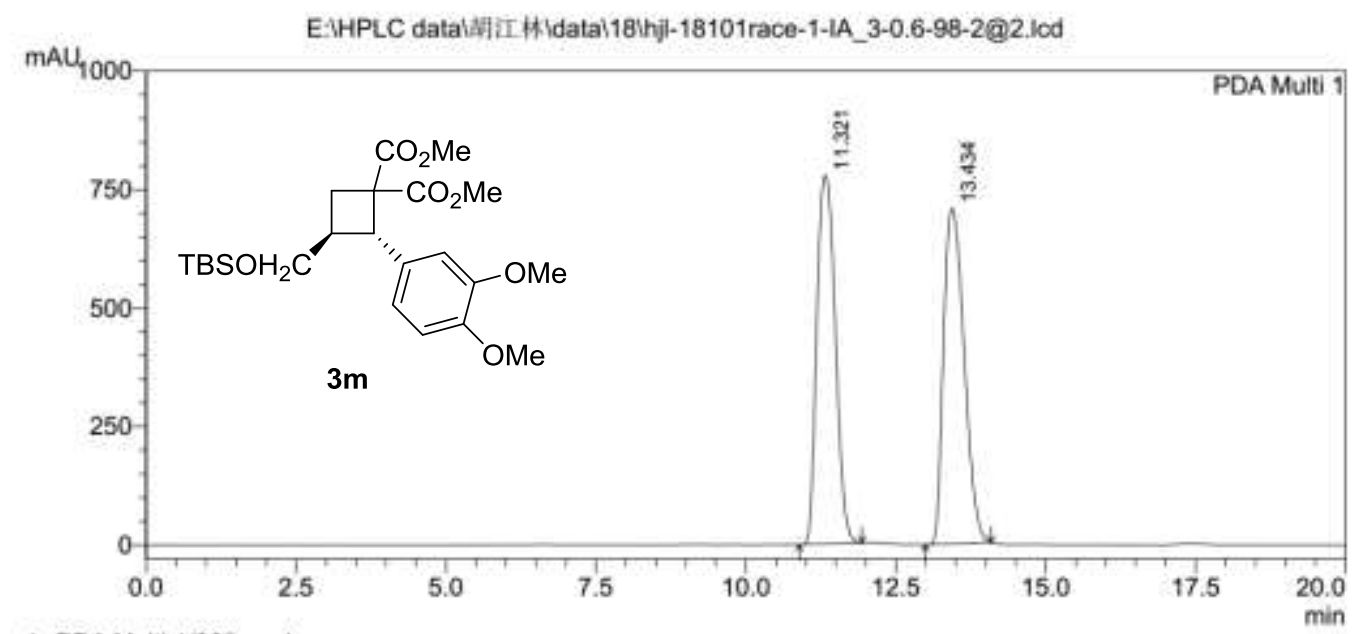

1 PDA Multi $1 / 280 \mathrm{~nm} 4 \mathrm{~nm}$

\section{<Results>}

PDA Ch1 $280 \mathrm{~nm} 4 \mathrm{~nm}$
\begin{tabular}{|l|l|l|r|r|r|r|}
\hline Peak \# & Ret. Time & \multicolumn{1}{c|}{ Area } & Height & \multicolumn{1}{c|}{ Area $\%$} & Height $\%$ & Mark \\
\hline 1 & 11.321 & 16618349 & 777928 & 49.649 & 52.340 & \\
\hline 2 & 13.434 & 16853079 & 708359 & 50.351 & 47.660 & \\
\hline Total & & 33471428 & 1486288 & 100.000 & 100.000 & \\
\hline
\end{tabular}

\section{$<$ Chromatogram $>$}

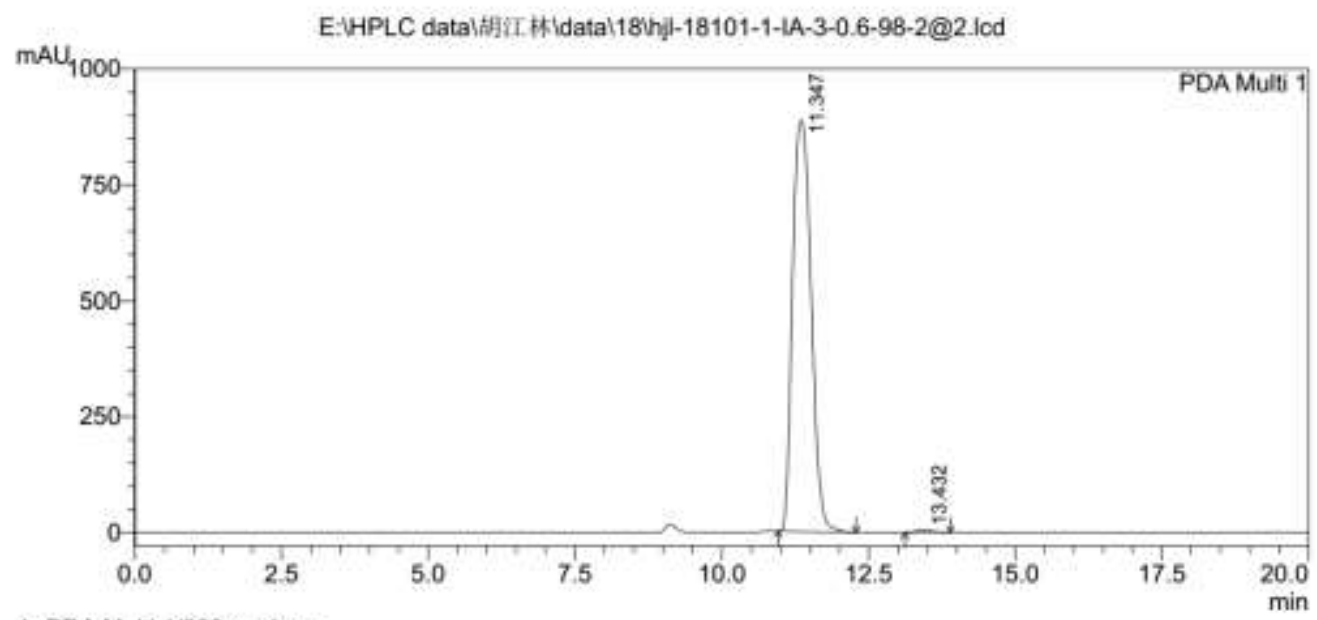

1 PDA Multi $1 / 280 \mathrm{~nm} 4 \mathrm{~nm}$

\section{<Results>}

PDA Ch1 280nm 4nm
\begin{tabular}{|l|r|r|r|r|r|r|}
\hline Peak \# & Ret. Time & \multicolumn{1}{c|}{ Area } & \multicolumn{1}{|c|}{ Height } & Area $\%$ & Height \% & Mark \\
\hline 1 & 11.347 & 19558353 & 887644 & 99.502 & 99.478 & $\mid$ \\
\hline 2 & 13.432 & 97955 & 4659 & 0.498 & 0.522 & 1000 \\
\hline rotal & & 19656308 & 892303 & 100.000 & 100.000 \\
\hline
\end{tabular}


$<$ Chromatogram $>$

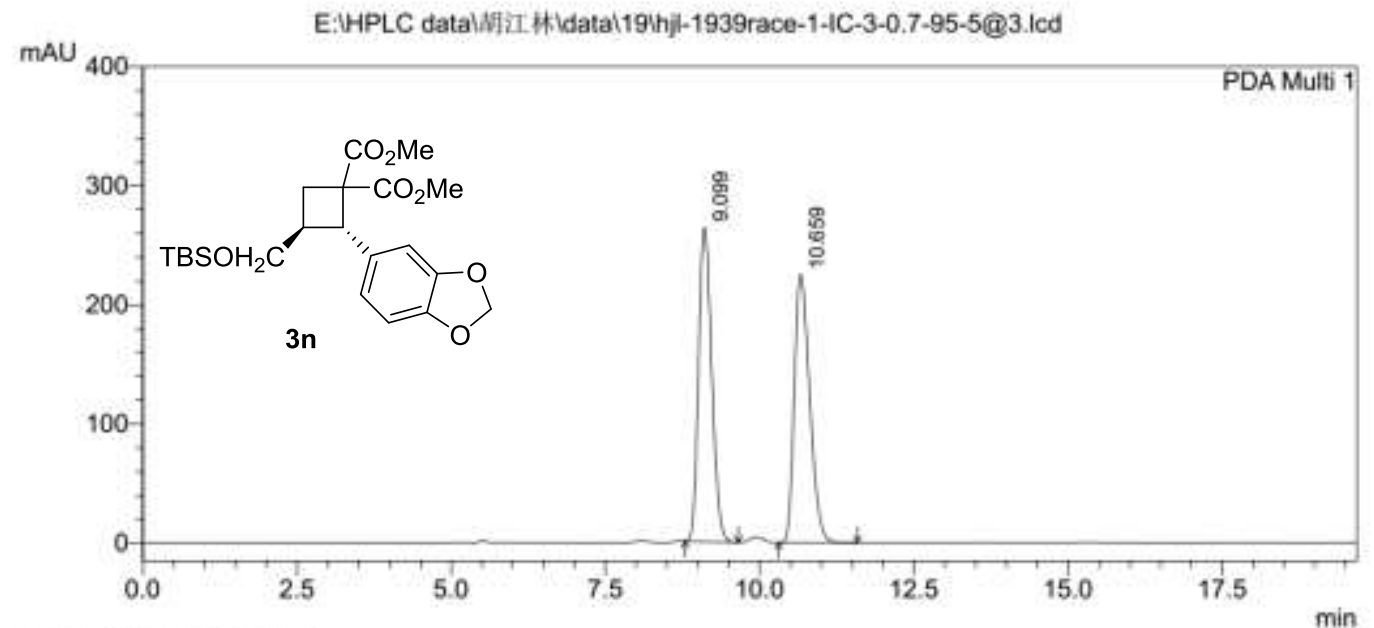

1 PDA Multi $1 / 300 \mathrm{~nm}$ 4nm

<Results>

PDA Ch1 300nm 4nm
\begin{tabular}{|l|r|r|r|r|r|r|r|}
\hline Peak \# & Ret. Time & \multicolumn{1}{|c|}{ Area } & Height & Area $\%$ & Height \% & Mark \\
\hline 1 & 9.099 & 3896571 & 263815 & 49.903 & 53.930 & \\
\hline 2 & 10.659 & 3911734 & 225369 & 50.097 & 46.070 & \\
\hline Total & & 7808305 & 489184 & 100.000 & 100.000 & \\
\hline
\end{tabular}

<Chromatogram>

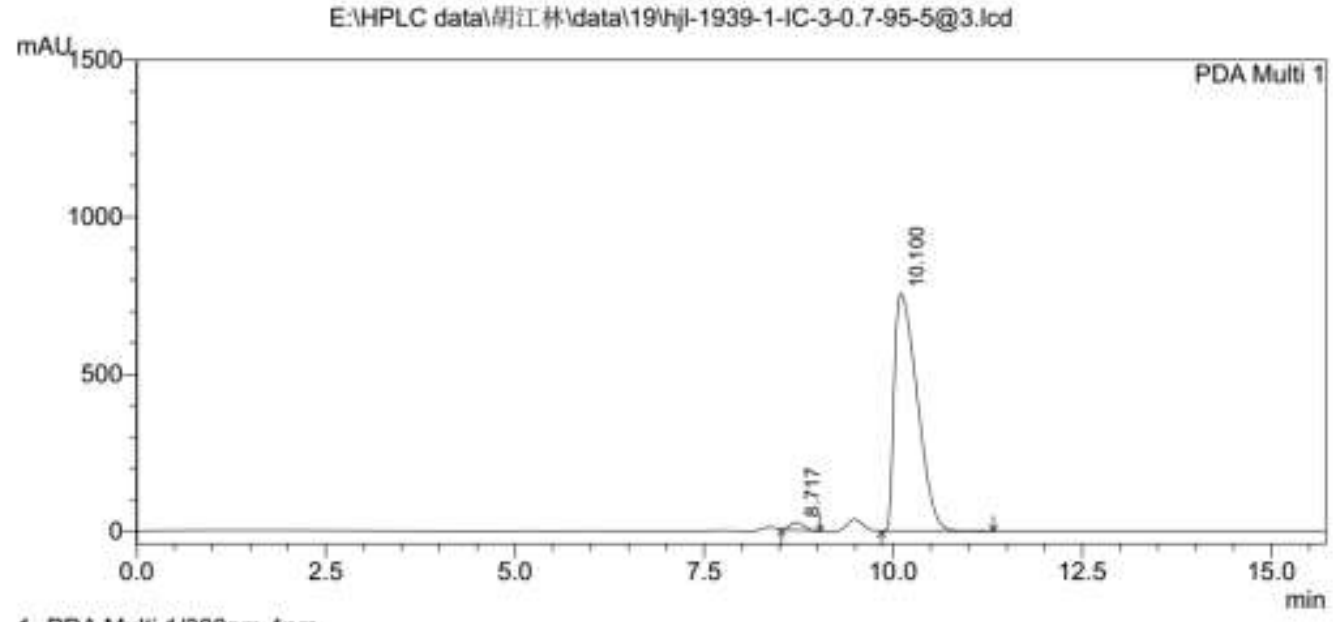

1 PDA Multi $1 / 300 \mathrm{~nm} 4 \mathrm{~nm}$

\section{<Results>}

PDA Ch1 300nm 4nm
\begin{tabular}{|l|r|r|r|r|r|r|}
\hline Peak \# & Ret. Time & \multicolumn{1}{|c|}{ Area } & Height & \multicolumn{1}{|c|}{ Area $\%$} & Height \% & Mark \\
\hline 1 & 8.717 & 314179 & 24819 & 1.928 & 3.177 & \\
\hline 2 & 10.100 & 15981535 & 756275 & 98.072 & 96.823 & $\mathrm{~S}$ \\
\hline Total & & 16295714 & 781094 & 100.000 & 100.000 & \\
\hline
\end{tabular}


<Chromatogram>

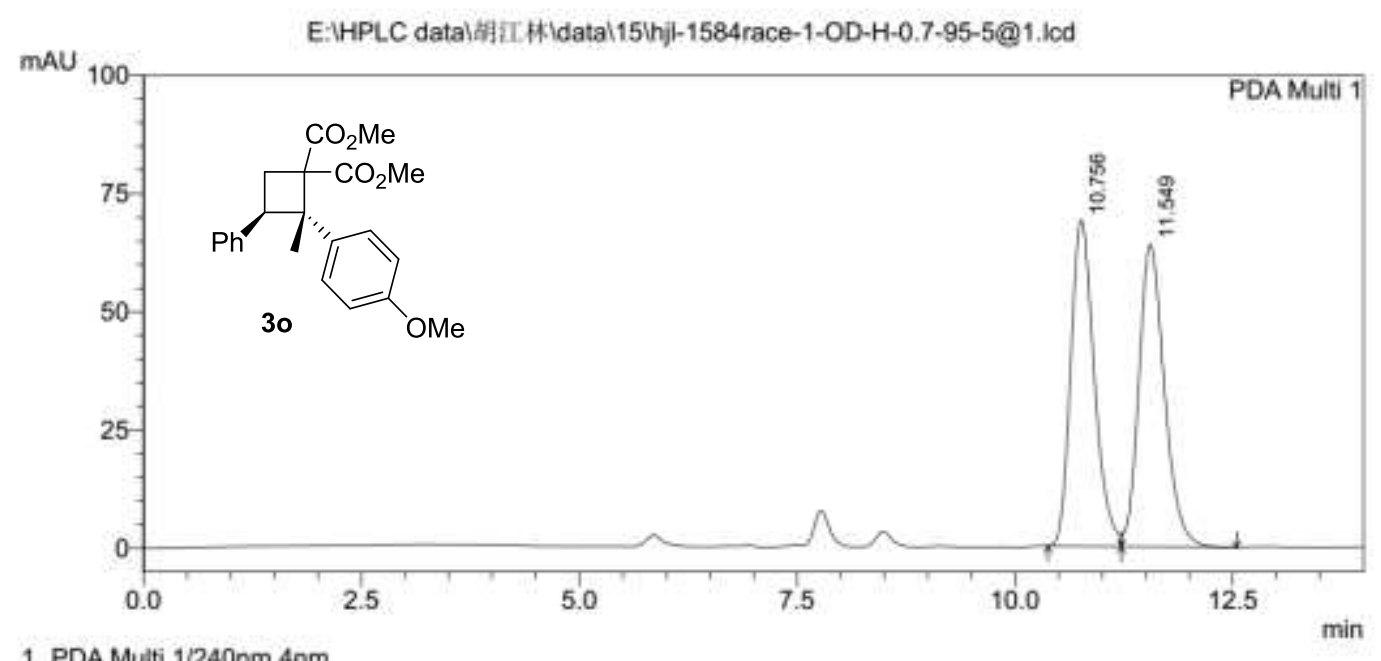

1 PDA Multi $1 / 240 \mathrm{~nm} 4 \mathrm{~nm}$

<Results>

PDA Ch1 240 nm 4nm
\begin{tabular}{|l|r|r|r|r|r|r|}
\hline Peak \# & Ret. Time & \multicolumn{1}{|c|}{ Area } & \multicolumn{1}{c|}{ Height } & Area $\%$ & \multicolumn{1}{|c|}{ Height \% } & Mark \\
\hline 1 & 10.756 & 1293150 & 69070 & 49.774 & 51.917 & \\
\hline 2 & 11.549 & 1304875 & 63969 & 50.226 & 48.083 & V \\
\hline Total & & 2598025 & 133039 & 100.000 & 100.000 & \\
\hline
\end{tabular}

$<$ Chromatogram>

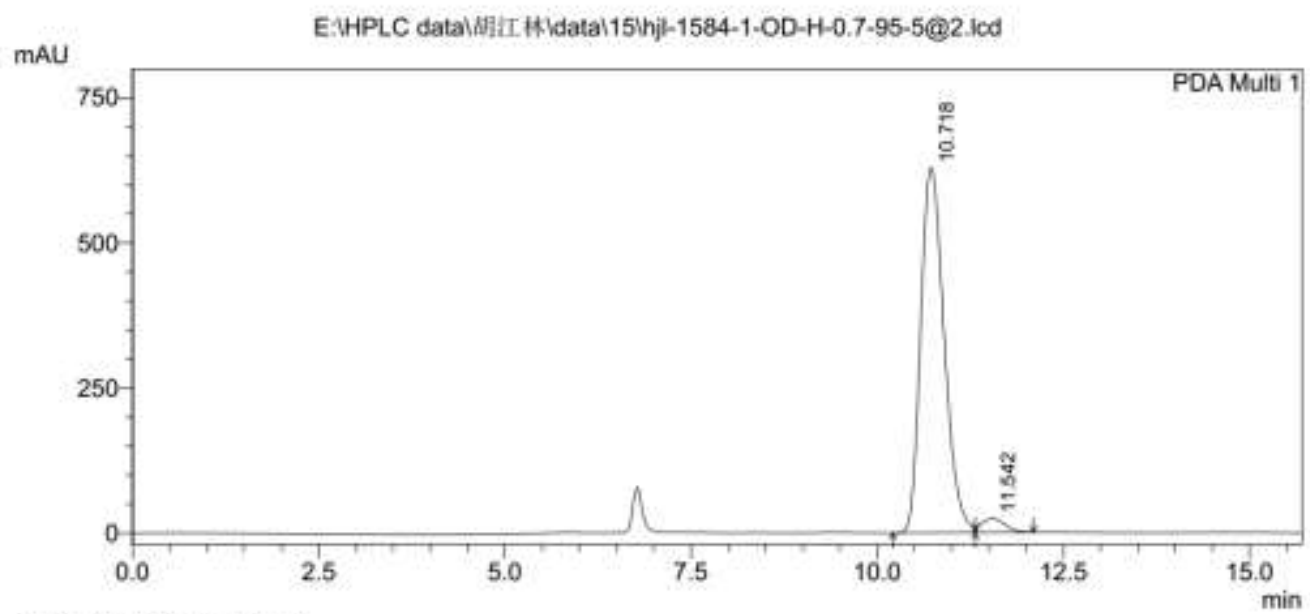

1 PDA Mutti $1 / 240 \mathrm{~nm} 4 \mathrm{~nm}$

\section{<Results>}

PDA Ch1 240nm 4nm
\begin{tabular}{|l|r|r|r|r|r|r|}
\hline Peak \# & Ret. Time & \multicolumn{1}{c|}{ Area } & \multicolumn{1}{c|}{ Height } & Area $\%$ & Height $\%$ & Mark \\
\hline 1 & 10.718 & 13764939 & 629473 & 96.243 & 96.299 & \\
\hline 2 & 11.542 & 537326 & 24189 & 3.757 & 3.701 & $\mathrm{~V}$ \\
\hline Total & & 14302265 & 653662 & 100.000 & 100.000 & \\
\hline
\end{tabular}


$<$ Chromatogram>

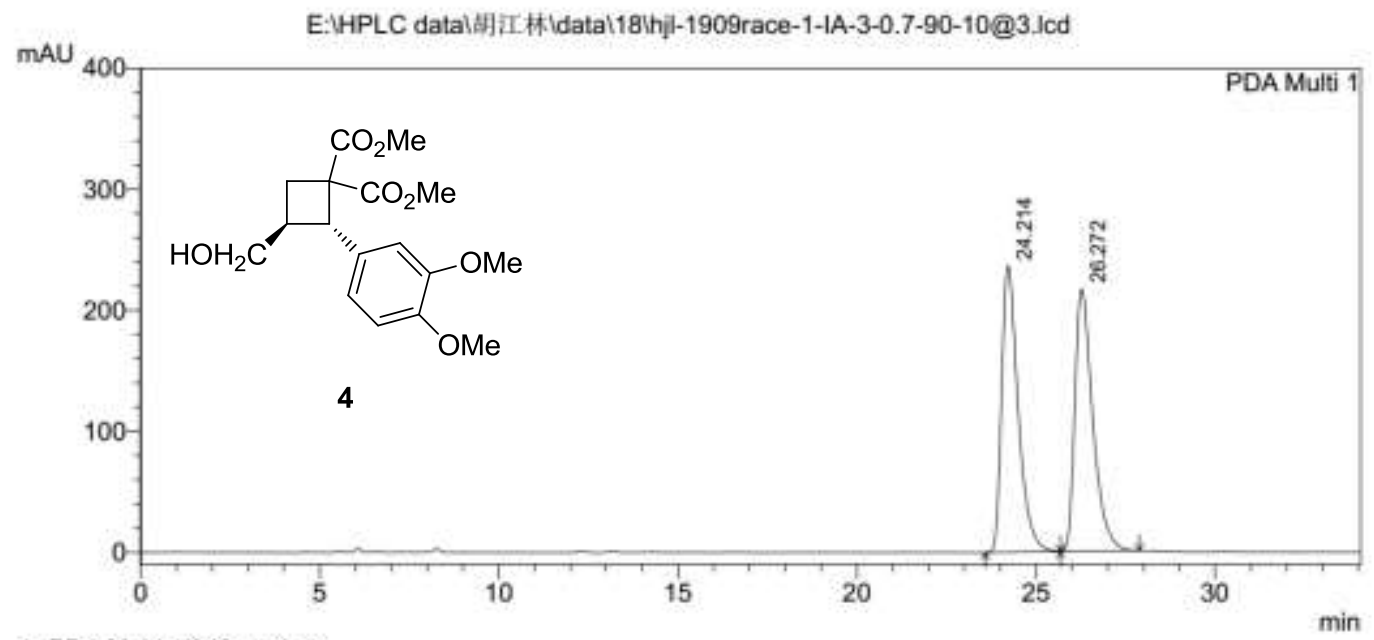

1 PDA Multi $1 / 240 \mathrm{~nm} 4 \mathrm{~nm}$

\section{<Results>}

PDA Ch1 $240 \mathrm{~nm}$ 4nm
\begin{tabular}{|l|r|r|r|r|r|r|}
\hline Peak \# & Ret. Time & \multicolumn{1}{|c|}{ Area } & Height & Area $\%$ & Height \% & Mark \\
\hline 1 & 24.214 & 7545041 & 236875 & 50.099 & 52.199 & \\
\hline 2 & 26.272 & 7515244 & 216919 & 49.901 & 47.801 & V \\
\hline Total & & 15060285 & 453794 & 100.000 & 100.000 & \\
\hline
\end{tabular}

$<$ Chromatogram>

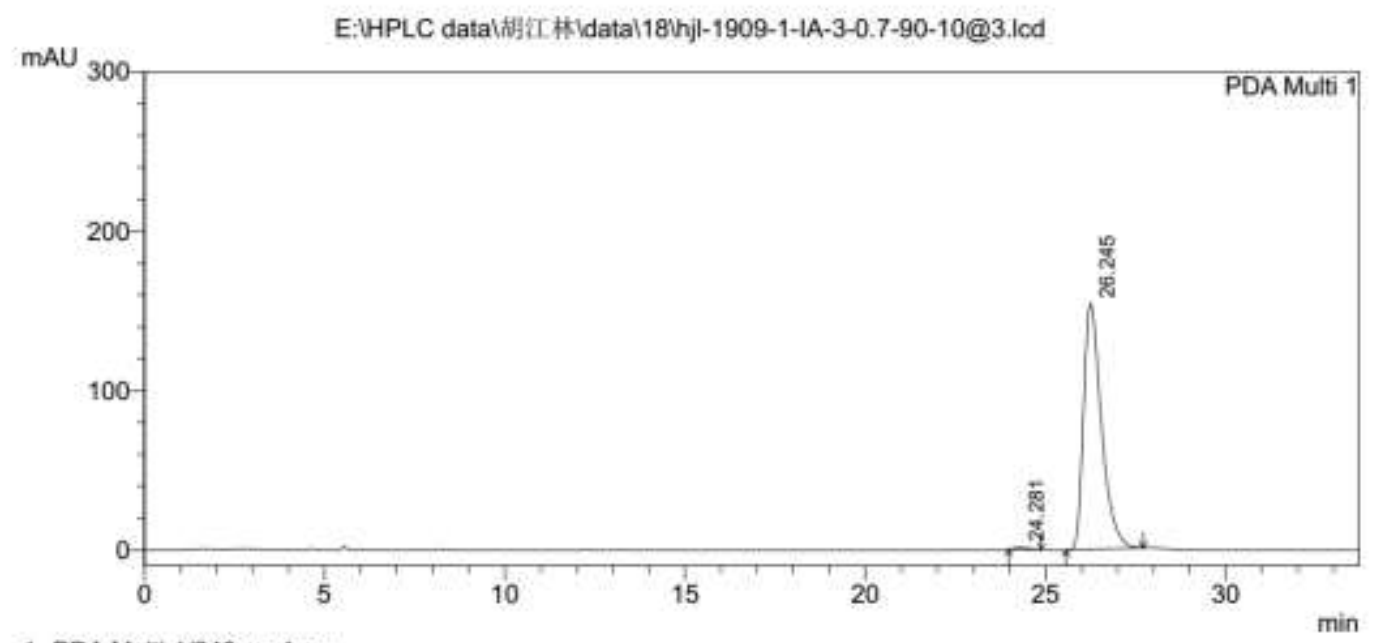

1 PDA Multi $1 / 240 \mathrm{~nm}$ 4nm

\section{$<$ Results>}

PDA Ch1 $240 \mathrm{~nm} 4 \mathrm{~nm}$
\begin{tabular}{|l|r|r|r|r|r|r|}
\hline Peak \# & Ret Time & \multicolumn{1}{c|}{ Area } & Height & Area $\%$ & Height $\%$ & Mark \\
\hline 1 & 24.281 & 37749 & 1436 & 0.705 & 0.922 & \\
\hline 2 & 26.245 & 5317757 & 154355 & 99.295 & 99.078 & \\
\hline Total & & 5355505 & 155791 & 100.000 & 100.000 \\
\hline
\end{tabular}


$<$ Chromatogram>

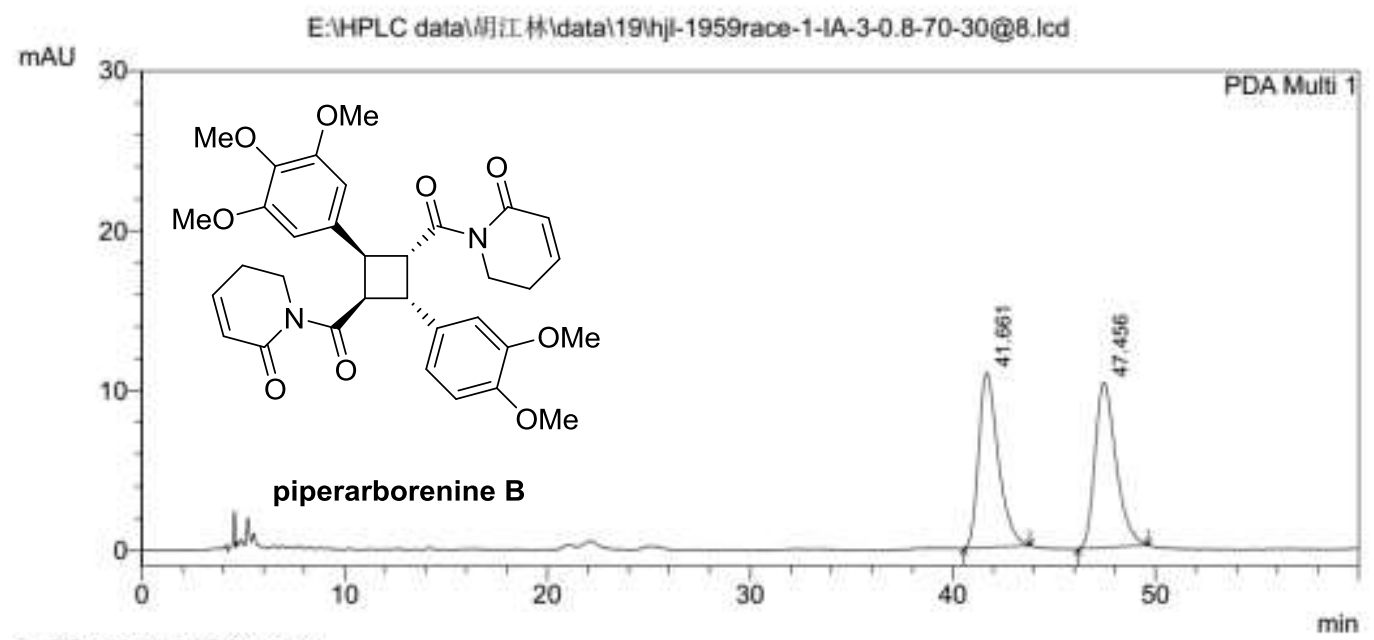

1 PDA Multi $1 / 254 \mathrm{~nm} 4 \mathrm{~nm}$

\section{<Results>}

PDA Ch1 $254 \mathrm{~nm} 4 \mathrm{~nm}$
\begin{tabular}{|l|r|r|r|r|r|r|}
\hline Peak \# & Ret. Time & \multicolumn{1}{|c|}{ Area } & Height & Area $\%$ & Height $\%$ & Mark \\
\hline 1 & 41.661 & 736700 & 10938 & 49.661 & 51.425 & \\
\hline 2 & 47.456 & 746765 & 10332 & 50.339 & 48.575 \\
\hline Total & & 1483465 & 21269 & 100.000 & 100.000 \\
\hline
\end{tabular}

\section{$<$ Chromatogram>}

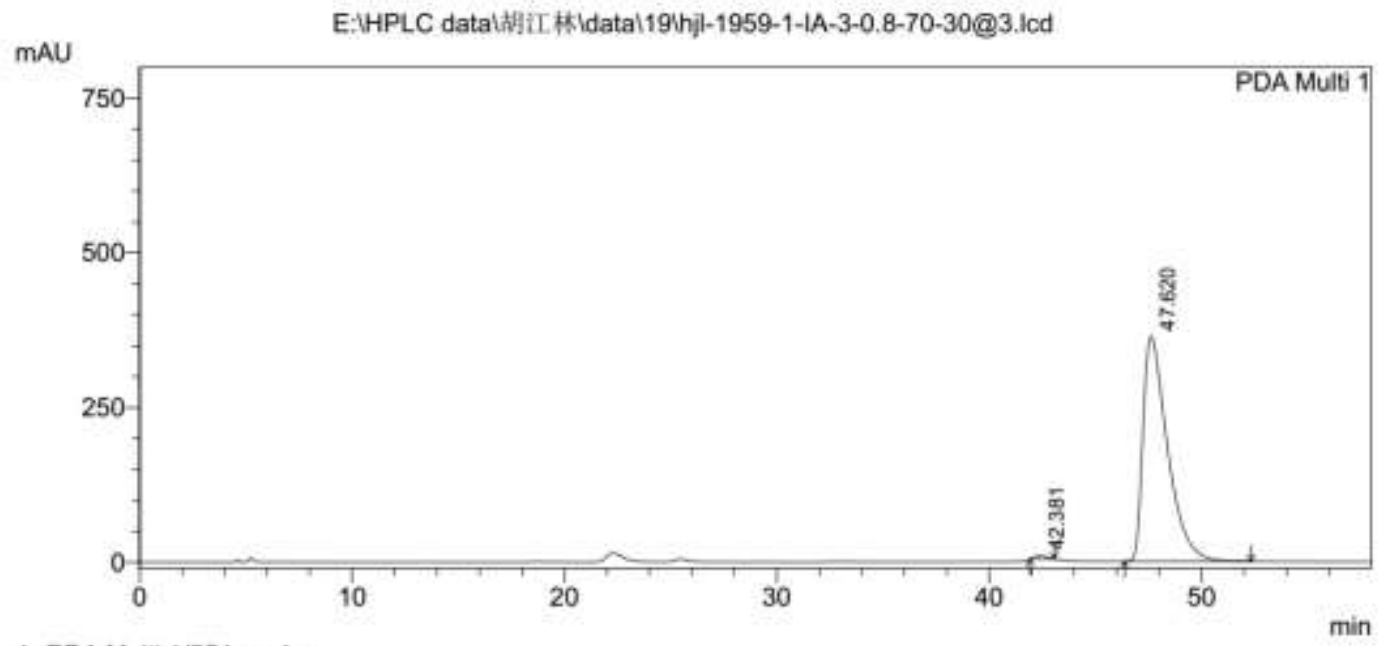

1 PDA Multi $1 / 254 \mathrm{~nm} 4 \mathrm{~nm}$

\section{<Results>}

PDA Ch1 $254 \mathrm{~nm} 4 \mathrm{~nm}$
\begin{tabular}{|l|r|r|r|r|r|r|}
\hline Peak \# & Ret. Time & \multicolumn{1}{c|}{ Area } & Height & Area $\%$ & Height $\%$ & Mark \\
\hline 1 & 42.381 & 211188 & 5128 & 0.716 & 1.389 & \\
\hline 2 & 47.620 & 29286759 & 363964 & 99.284 & 98.611 \\
\hline Total & & 29497947 & 369091 & 100.000 & 100.000 \\
\hline
\end{tabular}

